\title{
THE ELEMENTARY PROOF OF THE RIEMANN'S HYPOTHESIS
}

\author{
JAN FELIKSIAK
}

\begin{abstract}
This research paper aims to explicate the complex issue of the Riemann's Hypothesis and ultimately presents its elementary proof. The method implements one of the binomial coefficients, to demonstrate the maximal prime gaps bound. Maximal prime gaps bound constitutes a comprehensive improvement over the Bertrand's result, and becomes one of the key elements of the theory. Subsequently, implementing the theory of the primorial function and its error bounds, an improved version of the Gauss' offset logarithmic integral is developed. The integral serves as the Supremum bound of the prime counting function $\pi_{(n)}$. Due to its very high precision, it permits to verify the relationship between the prime counting function $\pi_{n}$ and the offset logarithmic integral of Carl Gauss'. The collective mathematical theory, via the Niels F. Helge von Koch [24] equation:$$
\pi_{(n)}=L i_{(n)}+\mathcal{O}(\sqrt{n} \log (n))
$$

enables to prove the Riemann's Hypothesis conclusively.

Revised May 2021

(C)2021 Jan Feliksiak

2010 Mathematics Subject Classification. 0102, 01A50, 05A10, 1102, 1103, 11A41, 11L20, $11 \mathrm{~N} 05$.

Key words and phrases. Cramér's conjecture, distribution of primes, elementary proof of the Riemann's hypothesis, Landau's problems, Legendre's Conjecture, Littlewood's proof 1914, logarithmic integral, maximal prime gaps, maximal prime gaps Upper Bound, prime counting function $\pi_{(n)}$, Prime Number Theorem, Skewes' problem, tailored logarithmic integral, the primorial function, the Infimum of $\pi_{(n)}$, the Supremum of $\pi_{(n)}$. 


\section{Contents}

1. Definitions section 3

1.1. Mathematical constants definitions 3

2. The binomial expansion $2^{\left(n+\mathcal{G}_{(n)}\right)} \quad 4$

2.1. Preliminaries 4

2.2. Bounds on the logarithm of the binomial coefficient 4

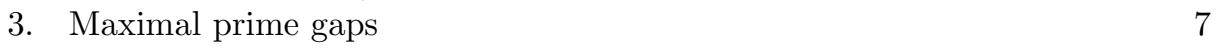

3.1. Maximal prime gaps standard measure 8

3.2. The conjectures of Cramér's and Legendre 11

4. Theory of the Primorial Function 12

4.1. Upper Bound on the logarithm of the primorial function 12

4.2. The estimation error bounds on the difference of $\left(p_{(n)}-\log p_{(n)} \sharp\right) \quad 14$

5. The prime counting function $\pi_{(n)}$ Supremum/Infimum Bounds 18

$\begin{array}{ll}\text { 5.1. The Tailored logarithmic integral definitions } & 19\end{array}$

$\begin{array}{ll}\text { 5.2. Preliminary theory } & 19\end{array}$

5.3. Tailored integral $T L i_{(n)}$ step sequence 20

5.4. Step sequence estimation error bounds 24

5.5. Supremum and Infimum estimation error bounds on $T L i_{(n)}-\pi_{(n)} \quad 30$

5.6. The elementary bounds on the prime counting function $\pi_{n} \quad 34$

5.7. Supremum of $\pi_{(n)}$ and the Skewes' $\pi_{(n)}>L i_{(n)}$ problem appraisal $\quad 40$

5.8. Estimation error bounds on the difference $T L i_{(n)}-\pi_{(n)} \quad 43$

6. Gauss' logarithmic integral $L i_{(n)}$ and Riemann's hypothesis 46

6.1. Divergence of the estimation error of $L i_{(n)} \quad 46$

6.2. $L i_{(n)}$ estimation error bounds $\quad 47$

6.3. Primary estimation error bound of $L i_{(n)} \quad 50$

6.4. Lower bound on the error term of the Gauss' logarithmic integral 51

6.5. Upper bound on the error term of the Gauss' logarithmic integral 52

6.6. Estimation error bounds $\left(L i_{(n)}-\pi_{(n)}\right)$ and $\left(T L i_{(n)}-\pi_{(n)}\right)$ summary 53

6.7. Riemann's hypothesis 53

References $\quad 55$

7. Appendix 57

7.1. Graphical comparison of functions $\quad 57$

7.2. Tabular data 64 


\section{Definitions SECtion}

Within the scope of the paper, prime gap of the size $\mathfrak{g} \in \mathbb{N} \mid \mathfrak{g} \geq 2$ is defined as an interval between two primes $\left(p_{i}, p_{i+1}\right]$, containing $(\mathfrak{g}-1)$ composite integers. Maximal prime gap of the size $\mathfrak{g}$, is a gap strictly exceeding in size any preceding gap. In this document, all computations pertaining to the logarithmic integral, were carried out using the Gauss' offset logarithmic integral : $\int_{2}^{n} \frac{d t}{\log t}$.

All calculations and graphing were carried out with the aid of Mathematica ${ }^{\circledR}$ software.

\subsection{Mathematical constants definitions.}

Definition 1.1 (Golden Mean).

$$
\mathcal{G M}=\frac{\sqrt{5}-1}{2} \approx 0.618033988749894848204586834365638117720309180
$$

Definition 1.2 (Lambda constant).

$$
\lambda=\left(\frac{14 \pi}{29}+\mathcal{G M}\right) \approx 2.1346649249656571012555181228453981307810116485
$$

Definition 1.3 (Khinchin's constant).

$$
K=\exp \left(\frac{1}{\log 2} \sum_{i=2}^{\infty} \frac{(-1)^{i}\left(2-2^{i}\right)}{i} \zeta_{(i)}^{\prime}\right) \approx 2.685452001065306445309714835482
$$

Definition 1.4 (Beta constant).

$$
\begin{array}{r}
\beta=(1+(\exp (1)-K)) \approx 1.0328298273937387900505726358708668039369 \\
\text { where } K \text { is the Khinchin's constant. }
\end{array}
$$

Definition 1.5 (Glaisher - Kinkelin constant).

$$
\mathcal{A}=\exp \left(\frac{1}{12}-\zeta^{\prime}(-1)\right) \approx 1.28242712910062263687534256886979172776768893
$$

Definition 1.6 (Double Twin primes constant).

$$
\mathcal{T C}=2 \prod_{p>2}\left(1-\frac{1}{(p-1)^{2}}\right) \approx 1.32032363169373914785562422002911155686525
$$


2. The BINOMial EXPANSION $2^{\left(n+\mathcal{G}_{(n)}\right)}$

\subsection{Preliminaries.}

Bertrand's Conjecture is a well known mathematical theorem concerning the size of the prime gaps. The first elementary proof of the Bertrands Conjecture regarding the existence of at least one prime within the interval from $n$ to $2 n$ was due to Srinivasa Ramanujan, who in 1919 presented his elegant proof. Paul Erdös at the age of 19 improved Ramanujan's proof in 1932. In his proof of the Bertrand's conjecture Paul Erdös utilized the largest binomial coefficient of the binomial expansion $2^{2 n}$ :

$$
\mathcal{N}=\left(\begin{array}{c}
2 n \\
n
\end{array}\right)=\left(\frac{(2 n) !}{(n ! \times n !)}\right)=\left(\frac{(n+1)(n+2) \cdots(2 n)}{n !}\right)
$$

The problem of existence of at least one prime within the interval from $n$ to $n+c=t$ is substantially more difficult than the Bertrand's Conjecture. The issue pertains to the considerably shorter interval length of the function $\mathcal{G}_{(n)}$, as compared to the interval of length $n$, pertinent to the research that both Srinivasa Ramanujan and Paul Erdös worked on.

One of the major step-stones of this paper is the comprehensively improved bound on the maximal prime gaps. This goal is achieved by an implementation of a binomial expansion coefficient pertinent to the function $\mathcal{G}_{(n)}$. Now, for all $n \in \mathbb{N} \mid n \geq 5$, we make the following definitions:

Definition 2.1 (Interval length). $c=\mathcal{G}_{(n)}=\left\lfloor 5\left(\log _{10} n\right)^{2}\right\rfloor$

Definition 2.2 (Interval endpoint). $t=\left(n+\mathcal{G}_{(n)}\right)=(n+c)$

The binomial coefficient $\mathcal{M}_{(t)}$ related to the current research is a part of the associated binomial expansion:

$$
2^{t}>>\left(\begin{array}{c}
n+c \\
n
\end{array}\right)
$$

Definition 2.3 (Binomial coefficient).

$$
\mathcal{M}_{(t)}=\left(\begin{array}{c}
n+c \\
n
\end{array}\right)=\left(\frac{(n+c) !}{(n ! c !)}\right)
$$

Definition 2.4 (Logarithm of the binomial coefficient).

$\log \mathcal{M}_{(t)}=\log \left(\frac{(n+c) !}{(n ! \times c !)}\right)=\log (t !)-\log (n !)-\log (c !)=\sum_{k=1}^{c} \log (n+k)-\sum_{k=1}^{c} \log k$

\subsection{Bounds on the logarithm of the binomial coefficient.}

Lemma 2.5 (Upper and Lower bounds on the log of $n$ !).

The bounds on the logarithm of $n !$ are given by:

$$
n \log (n)-n+1 \leq \log (n !) \leq(n+1) \log (n+1)-n \quad \forall n \in \mathbb{N} \mid n \geq 5
$$


Proof.

Evidently,

$$
\log (n !)=\sum_{k=1}^{n} \log (k) \quad \forall n \in \mathbb{N} \mid n \geq 2
$$

Now, the pertinent integrals to consider are:

$$
\int_{1}^{n} \log (x) d x \leq \log (n !) \leq \int_{0}^{n} \log (x+1) d x \quad \forall n \in \mathbb{N} \mid n \geq 5
$$

Accordingly, evaluating those integrals we obtain:

$$
\begin{aligned}
n \log (n)-n+1 \leq \log (n !) \leq n \log \left(\frac{(n+1)}{e}\right)+ & \log \left(\frac{(n+1)}{e}\right)+1 \\
& =(n+1) \log (n+1)-n
\end{aligned}
$$

Concluding the proof of Lemma 2.5 .

\section{Remark 2.1.}

Observe that $\log \mathcal{M}_{(t)}$ is a difference of logarithms of factorial terms:

$$
\log \mathcal{M}_{(t)}=(\log (t !)-\log (n !)-\log (c !))
$$

Consequently, implementing the lower/upper bounds on the logarithm of $n$ ! for the bounds on $\log \mathcal{M}_{(t)}$, results in bounds of the form:

$$
\log \left(\frac{(t+k)^{(t+k)}}{(n+k)^{(n+k)}(c+k)^{(c+k)}}\right) \quad \text { for } \forall k \in \mathbb{N} \cup\{0\}
$$

Keeping the values of $c, n$ and $t$ constant and letting the variable $k$ to increase unboundedly, results in an unbounded monotonically decreasing function. When implementing the lower/upper bounds on the logarithm of $n$ ! for the Supremum/Infimum bounds on $\log \mathcal{M}_{(t)}$, the variable $k$ appears only with values $k=\{0,1\}$ respectively. The combined effect of the difference of the logarithms of factorial terms in $\log \mathcal{M}_{(t)}$ and the decreasing property of the function 2.5 , imposes a reciprocal interchange of the bounds 2.1, when implementing them for the bounds on $\log \mathcal{M}_{(t)}$.

Lemma $2.6\left(\log \mathcal{M}_{(t)}\right.$ Supremum Bound). by:

The Supremum Bound on the logarithm of the binomial coefficient $\mathcal{M}_{(t)}$ is given

$$
\log \mathcal{M}_{(t)} \leq \log \left(\frac{t^{t}}{n^{n} c^{c}}\right)-1=\mathcal{U} \mathcal{B}_{(t)} \quad \forall n \in \mathbb{N} \mid n \geq 5
$$

Proof.

Evidently, by Lemma 2.5 we have:

$$
(n \log (n)-n+1) \leq \log (n !)
$$

Substituting from the inequality 2.7 into the Definition 2.4 we obtain:

$$
\begin{aligned}
& (\log (t !)-\log (n !)-\log (c !)) \\
& \leq((t \log (t)-t+1)-(n \log (n)-n+1)-(c \log (c)-c+1)) \\
& =t \log (t)-n \log (n)-c \log (c)-1=\log \left(\frac{t^{t}}{n^{n} c^{c}}\right)-1
\end{aligned}
$$


Consequently,

$$
\log \mathcal{M}_{(t)} \leq \log \left(\frac{t^{t}}{n^{n} c^{c}}\right)-1=\mathcal{U} \mathcal{B}_{(t)}
$$

The Supremum bound $\mathcal{U} \mathcal{B}_{(t)}$ produces an increasing, strictly monotone sequence in $\mathbb{R}$. At $n=5$, the difference $\mathcal{U} \mathcal{B}_{(t)}-\log \mathcal{M}_{(t)}$ attains 0.143365 and diverges as $n \rightarrow \infty$. Therefore, Lemma 2.6 holds as specified.

Lemma $2.7\left(\log \mathcal{M}_{(t)}\right.$ Infimum bound).

The Infimum Bound on the natural logarithm of the binomial coefficient $\mathcal{M}_{(t)}$ for all $n \in \mathbb{N} \mid n \geq 5$ is given by:

$$
\log \mathcal{M}_{(t)} \geq \log \left(\frac{(t+1)^{(t+1)}}{(n+1)^{(n+1)}(c+1)^{(c+1)}}\right)=\mathcal{L B}_{(t)}
$$

Proof.

From Lemma 2.5 we have:

$$
\log (n !) \leq n \log (n+1)-n+\log (n+1)
$$

Substituting from the inequality 2.11 into the Definition 2.4 we obtain:

$$
\begin{array}{r}
\geq t \log (t+1)-n \log (n+1)-c \log (c+1)+\log (t+1)-\log (n+1)-\log (c+1) \\
=\log \left(\frac{(t+1)^{t}}{(n+1)^{n}(c+1)^{c}}\right)+\log \left(\frac{(t+1)}{(n+1)(c+1)}\right)
\end{array}
$$

Consequently,

$$
\log \mathcal{M}_{(t)} \geq \log \left(\frac{(t+1)^{(t+1)}}{(n+1)^{(n+1)}(c+1)^{(c+1)}}\right)=\mathcal{L B}_{(t)}
$$

The Infimum bound $\mathcal{L B}_{(t)}$ produces an increasing, strictly monotone sequence in $\mathbb{R}$. At $n=5$, the difference $\log \mathcal{M}_{(t)}-\mathcal{L B}_{(t)}$ attains 0.455384 and diverges as $n \rightarrow \infty$. Therefore, Lemma 2.7 holds as specified.

Consequently, from Lemma 2.7 and 2.6 we have:

$$
\log \left(\frac{(t+1)^{(t+1)}}{(n+1)^{(n+1)}(c+1)^{(c+1)}}\right) \leq \log \mathcal{M}_{(t)} \leq \log \left(\frac{t^{t}}{n^{n} c^{c}}\right)-1
$$

Inequality 2.14 presents very well streamlined Supremum/Infimum bounds on the $\log \mathcal{M}_{(t)}$. 


\section{MAXIMAL PRIME GAPS}
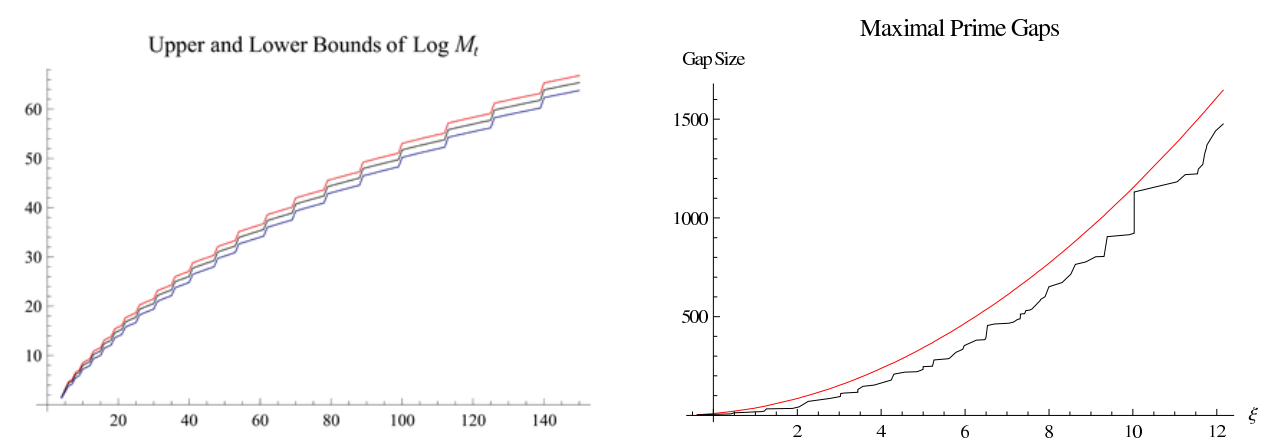

Figure 1. The left drawing shows the graphs of the lower (blue) and upper (red) bounds vs $\log \mathcal{M}_{(t)}$ (black). The right drawing shows the graph of $\mathcal{G}_{(n)}$ (red) and the actual maximal gaps (black) with respect to $\xi$ as given by the Definition 5.4. The graph has been produced on the basis of data obtained from C. Caldwell as well as from T. Nicely tables of maximal prime gaps.

From the Prime Number Theorem we have that an average gap between consecutive primes is given by $\log n$ for any $n \in \mathbb{N}$. There exist however prime gaps much shorter - containing only a single composite number, and gaps which are much longer than average - the maximal prime gaps. In 1929 R. Backlund [1] published a paper in which he proved the lower bound on the maximal prime gaps:

$$
p_{(n+1)}-p_{(n)}>(2-\epsilon) \log p_{(n)} \text { for any } \epsilon>0
$$

This was the first major result in this area. It had been improved upon in 1935 by Paul Erdös [14] who proved that:

$$
p_{(n+1)}-p_{(n)}>\frac{c\left(\log p_{(n)}\right) \log \left(\log p_{(n)}\right)}{\left(\log \left(\log \left(\log p_{(n)}\right)\right)\right)^{2}}
$$

However, it was the pioneering work of H. Cramér [12] using sophisticated probabilistic techniques, who attempted to establish the upper bound on the maximal prime gaps:

$$
p_{(n+1)}-p_{(n)} \leq\left(\log p_{(n)}\right)^{2}
$$

We begin with a preliminary derivation. Since the integers from 1 to $\mathrm{n}$ contain $\left\lfloor\frac{n}{p}\right\rfloor$ multiples of the prime number $\mathrm{p},\left\lfloor\frac{n}{p^{2}}\right\rfloor$ multiples of $p^{2}$ etc. Thus it follows that:

$$
n !=\prod_{p} p^{u_{(n, p)}} ; \text { where } u_{(n, p)}=\sum_{m \geq 1}\left\lfloor\frac{n}{p^{m}}\right\rfloor
$$

In accordance with the definitions 2.1 of $\mathcal{G}_{(n)}, 2.2$ of $t$ and 2.3 of $\mathcal{M}_{(t)}$ we obtain:

$$
\mathcal{M}_{(t)}=\prod_{p \leq t} p^{\mathcal{K}_{p}}
$$

where

$$
\mathcal{K}_{p}=\sum_{m=1}^{\infty}\left(\left\lfloor\frac{t}{p^{m}}\right\rfloor-\left\lfloor\frac{n}{p^{m}}\right\rfloor-\left\lfloor\frac{\mathcal{G}_{(n)}}{p^{m}}\right\rfloor\right)
$$


it follows that

$$
\mathcal{K}_{p} \leq\left\lfloor\frac{\log t}{\log p}\right\rfloor
$$

and so by the above, Lemma 2.6 and 2.7 we have:

$$
\mathcal{L B}_{(t)} \leq \log \mathcal{M}_{(t)}=\log \prod_{p \leq t} p^{\mathcal{K}_{p}}=\sum_{p \leq t} \mathcal{K}_{p} \log p \leq \mathcal{U} \mathcal{B}_{(t)} \quad \forall n \in \mathbb{N} \mid n \geq 5
$$

Where $\mathrm{p}$ is as usual a prime number. Let's define:

Definition 3.1. $s=\left\lfloor\frac{t}{2}\right\rfloor$

Lemma 3.2 (Prime Factors of $\mathcal{M}_{(t)}$ ).

The case when there does not exist any prime factor $p$ of $\mathcal{M}_{(t)}$ within the interval from $n$ to $\left(n+\mathcal{G}_{(n)}\right)=t$ for any $n \in \mathbb{N} \mid n \geq 8$, imposes an upper limit on all prime factors $p$ of $\mathcal{M}_{(t)}$. Consequently in this particular case, every prime factor $p$ must be less than or equal to $s=\left\lfloor\frac{t}{2}\right\rfloor$.

Proof.

Let $\mathrm{p}$ be a prime factor of $\mathcal{M}_{(t)}$ so that $\mathcal{K}_{p} \geq 1$ and suppose that every prime factor $p \leq n$. If

$$
s<p \leq n
$$

then,

$$
p<\left(n+\mathcal{G}_{(n)}\right)<2 p
$$

and

$$
p^{2}>\left(\frac{\left(n+\mathcal{G}_{(n)}\right)}{2}\right)^{2}>\left(n+\mathcal{G}_{(n)}\right)
$$

and so $\mathcal{K}_{p}=0$. Therefore $p \leq s$ for every prime factor $\mathrm{p}$ of $\mathcal{M}_{(t)}$, for any $n \in \mathbb{N} \mid n \geq 8$.

\subsection{Maximal prime gaps standard measure.}

The binomial coefficient $\mathcal{M}_{(t)}$ :

$$
\begin{array}{r}
2^{t / 2}<n^{\frac{c}{2}}<\exp \left(\mathcal{L B}_{(t)}\right) \leq \mathcal{M}_{(t)}=\left(\frac{(n+c) !}{(n ! \times c !)}\right) \leq \exp \left(\mathcal{U} \mathcal{B}_{(t)}\right)<n^{\frac{2 c}{3}}<2^{t} \\
\forall n \in \mathbb{N} \mid n \geq 22
\end{array}
$$

The bounds on the logarithm of $\mathcal{M}_{(t)}$ are given by Lemma 2.6 and 2.7:

$$
\begin{aligned}
& \mathcal{L B}_{(t)}=\log \left(\frac{(t+1)^{(t+1)}}{(n+1)^{(n+1)}(c+1)^{(c+1)}}\right) \\
& \leq \log \mathcal{M}_{(t)}=\sum_{k=1}^{c} \log (n+k)-\sum_{k=1}^{c} \log k \leq \log \left(\frac{t^{t}}{n^{n} c^{c}}\right)-1=\mathcal{U B}_{(t)} \\
& \forall n \in \mathbb{N} \mid n \geq 5
\end{aligned}
$$

\section{Remark 3.1.}

- The proof of the Maximal Gaps Theorem implements the Supremum bound function $\mathcal{U} \mathcal{B}_{\left(t_{s}\right)}$. Due to the fact that the Supremum function $\mathcal{U} \mathcal{B}_{(t)}$ applies values of $n, c$ and $t$ directly, it imposes a technical requirement to generate a set of pertinent values, to correctly approximate the interval $s$. This is to ascertain that the generated interval is at least equal or greater than $s$ as 
given by Definition 3.1, as well as the corresponding value of $c$. Respective definitions follow:

Definition 3.3. $n_{s}=\frac{n}{2}$

Definition 3.4. $c_{s}=5\left(\log _{10}\left(n_{s}\right)\right)^{2}+1$

Definition 3.5. $t_{s}=n_{s}+c_{s}$

- The function $\mathcal{G} s_{(n)}$ due to the implementation of the Floor function increases stepwise. The sudden increase in value of the function $\mathcal{G} s_{(n)}$ is mirrored by an analogous, simultaneous increase in both, implemented bounds on the function $\log \mathcal{M}_{(t)}$ as well as the function $\log \mathcal{M}_{(t)}$ itself.

Theorem 3.6 (Maximal Prime Gaps Bound and Infimum for primes).

For any $n \in \mathbb{N} \mid n \geq 8$ there exists at least one $p \in \mathbb{N} \mid n<p \leq n+\mathcal{G}_{(n)}=t$; where $p$ is as usual a prime number and the maximal prime gaps upper bound $\mathcal{G}_{(n)}$ is given by:

$$
\begin{aligned}
& \mathcal{G}_{(n)}=\left\lfloor 5\left(\log _{10} n\right)^{2}\right\rfloor \quad \forall n \in \mathbb{N} \mid n \geq 8 \\
& \text { Equivalently, } p_{i+1}-p_{i} \leq \mathcal{G}_{\left(p_{i}\right)}
\end{aligned}
$$

Proof.

Suppose that there is no prime within the interval from $n$ to $t$. Then in accordance with the hypothesis, by Lemma 3.2 we have that, every prime factor $p$ of $\mathcal{M}_{(t)}$ must be less than or equal to $s=\left\lfloor\frac{t}{2}\right\rfloor$. Invoking Definitions 3.3, 3.4 and 3.5, Lemma 2.6, 2.7 and the inequality 3.1 we obtain for all $n \in \mathbb{N} \mid n \geq 8$ :

$$
\begin{aligned}
& (3.4) \mathcal{L B}_{(t)}=\log \left(\frac{(t+1)^{(t+1)}}{(n+1)^{(n+1)}(c+1)^{(c+1)}}\right) \\
& \leq \log \mathcal{M}_{(t)}=\log \prod_{p \leq t_{(s)}} p^{\mathcal{K}_{p}}=\sum_{p \leq t_{(s)}} \mathcal{K}_{p} \log p \leq \log \left(\frac{\left(t_{s}\right)^{t_{s}}}{\left(n_{s}\right)^{n_{s}}\left(c_{s}\right)^{c_{s}}}\right)-1=\mathcal{U} \mathcal{B}_{\left(t_{s}\right)}
\end{aligned}
$$

In accordance with the hypothesis therefore, it must be true that:

$$
a_{c}=\log \left(\frac{(t+1)^{(t+1)}}{(n+1)^{(n+1)}(c+1)^{(c+1)}}\right)-\log \left(\frac{\left(t_{s}\right)^{t_{s}}}{\left(n_{s}\right)^{n_{s}}\left(c_{s}\right)^{c_{s}}}\right)+1<0
$$

Now, we apply the Cauchy's Root Test for $n \geq 43$ :

$$
\lim _{c \rightarrow \infty} \sqrt[c]{\left|a_{c}\right|}=\lim _{c \rightarrow \infty} \sqrt[c]{\left|\mathcal{L B}_{(t)}-\mathcal{U} \mathcal{B}_{\left(t_{s}\right)}\right|} \rightarrow 1
$$

At $n=43$ the Cauchy's Root Test attains $\approx 1.17851$ and tends asymptotically to 1 , decreasing strictly from above. Thus, by the definition of the Cauchy's Root Test, the series formed from the terms of the difference $\mathcal{L B}_{(t)}-\mathcal{U} \mathcal{B}_{\left(t_{s}\right)}$, diverges as $c$ increases unboundedly. Hence in accordance with the hypothesis, inequality 3.5 diverges to $-\infty$ as $n$ increases unboundedly. However, at $n=43$ the difference 3.5 attains $\sim 9.45885151$ and diverges as $n$ increases unboundedly. Thus, we have a contradiction to the initial hypothesis. This implies that for all $n \in \mathbb{N} \mid n \geq 43$ :

$$
\mathcal{L B}_{(t)}-\mathcal{U} \mathcal{B}_{\left(t_{s}\right)}>0
$$


Necessarily therefore, there must be at least one prime within the interval $c$ for all $n \in \mathbb{N} \mid n \geq 43$. Table 1 lists all values of $n$ s.t. $8 \leq n \leq 47$. Evidently, every possible sub-interval contains at least one prime number. Thus we deduce that Theorem 3.6 holds in this range as well. Consequently Theorem 3.6 holds as stated for all $n \in \mathbb{N} \mid n \geq 8$, thus completing the proof.

TABLE 1. Low range $\mathcal{G}_{(n)}$ vs primes within the range

\begin{tabular}{|c|c|c|c|c|c|}
\hline$n$ & $\mathcal{G}_{(n)}$ & primes & $n$ & $\mathcal{G}_{(n)}$ & primes \\
\hline 8 & 4 & 11 & 29 & 10 & 31,37 \\
\hline 11 & 5 & 13 & 31 & 11 & 37,41 \\
\hline 13 & 6 & 17,19 & 37 & 12 & $41,43,47$ \\
\hline 17 & 7 & 19,23 & 41 & 13 & $43,47,53$ \\
\hline 19 & 8 & 23 & 43 & 13 & 47,53 \\
\hline 23 & 9 & 29,31 & 47 & 13 & 53,59 \\
\hline
\end{tabular}

\section{Remark 3.2.}

From now on, we may relax the function $\mathcal{G}_{(n)}$, by dropping the floor function.

A stronger version of the Maximal Prime Gaps bound can be proven by analogous method. However, in most instances the bound provided by Theorem 3.6 is sufficient. For the sake of completeness, Theorem 3.7 specifies the sharper, enhanced bound:

Theorem 3.7 (Sharper Maximal Prime Gaps Supremum Bound).

For any $n \in \mathbb{N} \mid n \geq 11$ there exists at least one $p \in \mathbb{N} \mid n<p \leq n+\mathcal{G} s_{(n)}=t$; where $p$ is as usual a prime number and the maximal prime gaps standard measure $\mathcal{G}_{(n)}$ is given by:

$$
\begin{array}{r}
\mathcal{S U P}=\mathcal{G} s_{(n)}=\left\lfloor 5\left(\log _{10} n\right)^{2}-\frac{15}{8}\left(\log _{10} n\right)\right\rfloor \quad \forall n \in \mathbb{N} \mid n \geq 11 \\
\text { Equivalently, } p_{i+1}-p_{i} \leq \mathcal{G} s_{\left(p_{i}\right)}
\end{array}
$$

Proof.

For a proof please consult Feliksiak [18]. 


\subsection{The conjectures of Cramér's and Legendre.}

Corollary 3.8 (Cramér's Conjecture).

There exist at least one prime $p \in \mathbb{N}\left|n<p \leq\left(n+(\log n)^{2}\right) \forall n \in \mathbb{N}\right| n \geq 8$.

Proof. Since,

By Theorem 3.6 we have that there exist at least one prime $p \in \mathbb{N} \mid n<p \leq t$.

$$
\mathcal{G}_{(n)}=5\left(\log _{10} n\right)^{2}<\left((\log 10)\left(\log _{10} n\right)\right)^{2} \quad \forall n \in \mathbb{N} \mid n \geq 8
$$

Therefore the Cramér's Maximal Gaps Conjecture follows ipso facto.

Corollary 3.9 (Legendre's Conjecture).

There exist at least one prime $p \in \mathbb{N}\left|n^{2}<p \leq(n+1)^{2} \forall n \in \mathbb{N}\right| n \geq 2$

Proof.

Suppose that Theorem 3.9 is false for some $n \in \mathbb{N} \mid n>10$. This implies that

$$
\pi_{n^{2}}=\pi_{(n+1)^{2}}
$$

By Theorem 3.6 we have that

$$
\pi_{n^{2}}<\pi_{\left(n^{2}+\mathcal{G}_{\left(n^{2}\right)}\right)} \forall n \in \mathbb{N} \mid n \geq 8
$$

Therefore, in accordance with the hypothesis it must be true that:

$$
(n+1)^{2}<\left(n^{2}+\mathcal{G}_{\left(n^{2}\right)}\right)
$$

Thus,

$$
\frac{2 n+1}{5\left(\log _{10} n^{2}\right)^{2}}<1
$$

However, for any $n \in \mathbb{N} \mid n>10$, the limit of 3.9 by the L'Hôpital's rule is:

$$
\lim _{n \rightarrow \infty} \frac{2 n+1}{5\left(\log _{10} n^{2}\right)^{2}}=\lim _{n \rightarrow \infty} \frac{n(\log 10)^{2}}{20} \rightarrow \infty
$$

Hence the ratio 3.9 increases unboundedly as $\mathrm{n}$ tends to infinity. At $n=10$, the value of the inequality 3.9 equals 1.05 . It implies that:

$$
(n+1)^{2}>\left(n^{2}+\mathcal{G}_{\left(n^{2}\right)}\right) \quad \forall n \in \mathbb{N} \mid n \geq 10
$$

Hence we have a contradiction to the initial hypothesis. Consequently, Theorem 3.9 is satisfied for all $n \in \mathbb{N} \mid n \geq 10$. For all $n \in \mathbb{N} \mid 2 \leq n<10$ a simple computer verification shows that Theorem 3.9 holds in this range as well, thus concluding the proof. 


\section{Theory of the Primorial Function}

\subsection{Upper Bound on the logarithm of the primorial function.}

The natural logarithm of the primorial function is a key element of the definition of the tailored logarithmic integral. It paves the way for the estimation of the prime counting function $\pi_{(n)}$ with unparalleled accuracy. First, we define the primorial function for all $k \in \mathbb{N}$ :

Definition 4.1. $p_{k} \sharp=\prod_{i=1}^{k}\left(p_{i}\right)$

Definition 4.2. $\log \left(p_{k} \sharp\right)=\log \left(\prod_{i=1}^{k}\left(p_{i}\right)\right)=\sum_{i=1}^{k}\left(\log p_{i}\right)$

Lemma 4.3 (Upper Bound on the logarithm of the primorial).

The natural logarithm of the primorial function is strictly less than the respective prime number $p \in \mathbb{N}$ :

(4.1) $\log p_{(n)} \sharp<p_{(n)} \leq n \quad \forall n \in \mathbb{N} \mid n \geq 2$, where $p_{n}$ is the largest prime $p \leq n$ In particular the natural logarithm of the primorial function is asymptotic (from below) to the respective prime number:

$$
\log p_{(n)} \sharp \sim p_{(n)}
$$

For the purpose of the proof we may assume that the twin primes continue indefinitely, the proof validity will not be affected by this. This issue will be expounded on in the Remark 4.2 below.

Proof.

From the inequality 4.1 we have:

$$
p_{(n)} \sharp<\exp \left(p_{(n)}\right) \leq \exp (n) \quad \forall n \in \mathbb{N} \mid n \geq 2
$$

Since prime numbers continue indefinitely, both $p_{(n)} \sharp$ and $\exp \left(p_{(n)}\right)$, are monotonically increasing divergent sequences of positive real numbers for all $n \in \mathbb{N} \mid n \geq 2$ Suppose that Lemma 4.3 is false, in accordance with the hypothesis it implies that:

$$
\exp \left(p_{(n)}\right)-p_{(n)} \sharp<0
$$

However, at $p_{n}=13$ the difference 4.4 attains $\sim 412383.39201$ and further diverges exponentially. Therefore, we apply the d'Alemberts Ratio Test. Define a sequence for all prime numbers $p_{(n-1)}, p_{(n)} \in \mathbb{N} \mid p_{(n)} \geq 13$ :

Definition 4.4. $a_{(n)}=\frac{\exp \left(p_{(n)}\right)-p_{(n)} \sharp}{\exp \left(p_{(n-1)}\right)-p_{(n-1)} \sharp}$

Remark 4.1. The sequence $a_{(n)}$ given by the Definition 4.4, has the least value at the twin primes as the difference $p_{(n)}-p_{(n-1)}=2$. Consequently, it is therefore both necessary and sufficient, to consider the sequence $a_{(n)}$ at the twin prime numbers only, with $p_{(n)}=6 i+7 \mid i \in \mathbb{N}, i \geq 1$.

At the twin primes:

$$
\exp \left(p_{(n)}\right)=\exp \left(p_{(n-1)}+2\right)=\exp \left(p_{(n-1)}\right) \times \exp (2)
$$

Further,

$$
p_{(n)} \sharp=p_{(n-1)} \sharp\left(p_{(n)}\right)
$$


Thus, at the twin primes the sequence $a_{(n)}$ equals:

$$
a_{(n)}=\frac{\exp \left(p_{(n)}\right)-p_{(n)} \sharp}{\exp \left(p_{(n-1)}\right)-p_{(n-1)} \sharp}=\exp (2) \times\left[\frac{\left(\exp \left(p_{(n-1)}\right)\right)-\left(p_{(n-1)} \sharp\right) \frac{p_{(n)}}{\exp (2)}}{\exp \left(p_{(n-1)}\right)-p_{(n-1)} \sharp}\right]
$$

The bracketed expression on the RHS, at the twin primes approaches the limit:

$$
\lim _{n \rightarrow \infty}\left[\frac{\left(\exp \left(p_{(n-1)}\right)\right)-\left(p_{(n-1)} \sharp\right) \frac{p_{(n)}}{\exp (2)}}{\exp \left(p_{(n-1)}\right)-p_{(n-1)} \sharp}\right] \rightarrow 1
$$

at the twin primes therefore, the sequence $a_{(n)}$ must clearly approach the limit:

$$
\lim _{n \rightarrow \infty} a_{(n)}=\lim _{n \rightarrow \infty}\left[\exp (2) \times\left(\frac{\left(\exp \left(p_{(n-1)}\right)\right)-\left(p_{(n-1)} \sharp\right) \frac{p_{(n)}}{\exp (2)}}{\exp \left(p_{(n-1)}\right)-p_{(n-1)} \sharp}\right)\right] \exp (2)
$$

By the d'Alemberts Ratio Test, the series formed from the terms of the difference $\exp \left(p_{(n)}\right)-p_{(n)} \sharp$, diverges as $p_{(n)}$ increases unboundedly. Thus, it logically follows that:

$$
p_{(n)} \sharp \leq \exp \left(p_{(n)}\right) \quad \forall p_{(n)} \in \mathbb{N} \mid p_{(n)} \geq 13
$$

Necessarily therefore, we have a contradiction to the initial hypothesis. Since at the twin primes the sequence $\exp \left(p_{(n)}\right)-p_{(n)} \sharp$ approaches:

$$
\left[\exp \left(p_{(n)}\right)-p_{(n)} \sharp\right] \sim \exp (2)\left[\exp \left(p_{(n-1)}\right)-p_{(n-1)} \sharp\right]
$$

Rearranging the above, we obtain that at the twin primes the primorial approaches:

$$
p_{(n)} \sharp \sim \exp \left(p_{(n)}\right)-\exp (2)\left[\exp \left(p_{(n-1)}\right)-p_{(n-1)} \sharp\right]
$$

This in turn implies that a strict inequality holds:

$$
p_{(n)} \sharp<\exp \left(p_{(n)}\right)
$$

Since increasing the gap between the consecutive primes has the effect of exponentially increasing the value that the sequence $a_{(n)}$ attains, therefore this result holds for all $p_{(n)} \in \mathbb{N} \mid p_{(n)} \geq 13$. By taking the logarithms, we obtain:

$$
\log \left(p_{(n)} \sharp\right)<p_{(n)} \quad \forall p_{(n)} \in \mathbb{N} \mid p_{(n)} \geq 13
$$

Thus, Lemma 4.3 holds for all $p_{(n)} \in \mathbb{N} \mid p_{(n)} \geq 13$. Direct computation verifies that Lemma 4.3 holds for all $p_{(n)} \in \mathbb{N} \mid 2 \leq p_{(n)} \leq 13$. Therefore, Lemma 4.3 holds as stated:

$$
\log \left(p_{(n)} \sharp\right)<p_{(n)} \leq n \quad \forall n \in \mathbb{N} \mid n \geq 2
$$

Consequently, this implies that the sequence of the natural logarithm of the primorial function is asymptotic from below:

$$
\log \left(p_{(n)} \sharp\right) \sim p_{(n)}
$$

Concluding the proof of Lemma 4.3.

Lemma 4.3 also implies that:

$$
\sqrt[\left(p_{(n)}\right)]{\left(p_{(n)} \sharp\right)}<\sqrt[(p(n))]{\exp \left(p_{(n)}\right)}=\exp (1) \quad \forall p_{(n)} \in \mathbb{N}
$$


By the PNT, (Ruiz, 1997; Finch, 2003), and Lemma 4.3 we obtain therefore:

$$
\lim _{n \rightarrow \infty}\left(\sqrt\left[(p(n)]{\left(p_{(n)} \sharp\right)}\right) \rightarrow \exp (1)\right.
$$

Remark 4.2. The sequence:

$$
a_{(n)}=\frac{\exp \left(p_{(n)}\right)-p_{(n)} \sharp}{\exp \left(p_{(n-1)}\right)-p_{(n-1)} \sharp}
$$

as it has been demonstrated for the twin primes example in the proof of Lemma 4.3; for primes such that $\left(p_{(n)}-p_{(n-1)}=d \mid d \in \mathbb{N}\right)$ for some given particular $d$, the sequence $a_{(n)}$ at the respective prime pairs, converges to the limit:

$$
\lim _{n \rightarrow \infty} a_{(n)}=\lim _{n \rightarrow \infty}\left[\frac{\exp \left(p_{(n)}\right)-p_{(n)} \sharp}{\exp \left(p_{(n-1)}\right)-p_{(n-1)}}\right] \rightarrow \exp (d)
$$

The approximation improves rapidly as $p_{(n)}$ increases. This is the reason why the validity of the twin primes conjecture is not essential.

\subsection{The estimation error bounds on the difference of $\left(p_{(n)}-\log p_{(n)} \sharp\right)$.}

Lemma 4.5 (Lower Estimation Error Bound On The Difference $\left.p_{n}-\log p_{n} \sharp\right)$.

The error of estimation of the primorial function by the use of the value of $p_{(n)}$ imposes the following lower bound:

$$
\begin{gathered}
\mathcal{L B}_{p_{(n)}}=(\sqrt{5}-1)\left(4 \gamma^{2}-2 \gamma\right)\left(\log p_{(n)}\right) \sqrt[3]{p_{(n)}}<\left(p_{(n)}-\log p_{(n)} \sharp\right) \\
\forall p_{(n)} \in \mathbb{N} \mid p_{(n)} \geq 2
\end{gathered}
$$

where $\gamma \approx 0.57721566490153286060651209$ is the Euler-Mascheroni constant.

Proof.

Both $\exp \left(p_{(n)}\right)$ and $p_{(n)} \sharp$ as well as $\exp \left(\mathcal{L B}_{p_{(n)}}\right)$ are monotone, divergent sequences of positive real numbers for all $p_{(n)} \in \mathbb{N} \mid p_{(n)} \geq 2$. Suppose that Lemma 4.5 is false. From inequality 4.21 therefore, in accordance with the hypothesis we derive:

$$
\exp \left(p_{(n)}\right)-\left(p_{(n)} \sharp\right) \exp \left(\mathcal{L B}_{p_{(n)}}\right)<0
$$

However, at $p_{n}=13$ the difference 4.22 attains $\sim 328977.240182$ and further diverges exponentially. Therefore we apply the d'Alemberts Ratio Test. Define a sequence for all prime numbers $p_{(n-1)}, p_{(n)} \in \mathbb{N} \mid p_{(n)} \geq 13$ :

Definition 4.6. $a_{(n)}=\frac{\exp \left(p_{(n)}\right)-\left(p_{(n)} \sharp\right) \exp \left(\mathcal{L B}_{p_{(n)}}\right)}{\exp \left(p_{(n-1)}\right)-\left(p_{(n-1)} \sharp\right) \exp \left(\mathcal{L B}_{p_{(n-1)}}\right)} \quad \forall p_{(n)} \in \mathbb{N} \mid p_{(n)} \geq 13$

Remark 4.3. The terms of the sequence $a_{(n)}$ given by the Definition 4.6 have the least value at the twin primes since the difference $p_{(n)}-p_{(n-1)}=2$. Consequently, it is both necessary and sufficient, to consider the sequence 4.6 at the twin primes only, with $p_{(n)}=6 i+7 \mid i \in \mathbb{N}, i \geq 1$.

At the twin primes:

$$
\exp \left(p_{(n)}\right)=\exp \left(p_{(n-1)}+2\right)=\exp \left(p_{(n-1)}\right) \times \exp (2)
$$


Further:

$$
p_{(n)} \sharp=p_{(n-1)} \sharp\left(p_{(n)}\right)
$$

Thus, at the twin primes the sequence $a_{(n)}$ equals:

$$
a_{(n)}=\exp (2) \times\left[\frac{\left(\exp \left(p_{(n-1)}\right)\right)-\left(p_{(n-1)} \sharp\right) \exp \left(\mathcal{L B}_{p_{(n)}}\right) \frac{p_{(n)}}{\exp (2)}}{\exp \left(p_{(n-1)}\right)-p_{(n-1)} \sharp \exp \left(\mathcal{L B}_{p_{(n-1)}}\right)}\right]
$$

The bracketed expression on the RHS, at the twin primes approaches the limit:

$$
\lim _{n \rightarrow \infty}\left[\frac{\left(\exp \left(p_{(n-1)}\right)\right)-\left(p_{(n-1)} \sharp\right) \exp \left(\mathcal{L B}_{p_{(n)}}\right) \frac{p_{(n)}}{\exp (2)}}{\exp \left(p_{(n-1)}\right)-p_{(n-1)} \sharp \exp \left(\mathcal{L B}_{p_{(n-1)}}\right)}\right] \rightarrow 1
$$

at the twin primes therefore, the sequence $a_{(n)}$ must clearly approach the limit:

$$
=\lim _{n \rightarrow \infty}\left[\exp (2) \times\left(\frac{\left(\exp \left(p_{(n-1)}\right)\right)-\left(p_{(n-1)} \sharp\right) \exp \left(\mathcal{L B}_{p_{(n)}}\right) \frac{p_{(n)}}{\exp (2)}}{\exp \left(p_{(n-1)}\right)-p_{(n-1)} \sharp \exp \left(\mathcal{L B}_{p_{(n-1)}}\right)}\right)\right] \exp (2)
$$

By d'Alemberts Ratio Test, the series formed from the terms of the difference $\exp \left(p_{(n)}\right)-p_{(n)} \sharp \exp \left(\mathcal{L B}_{p_{(n)}}\right)$ diverges, as $p_{(n)}$ increases unboundedly. Thus, it logically follows that:

$$
p_{(n)} \sharp \exp \left(\mathcal{L B}_{p_{(n)}}\right) \leq \exp \left(p_{(n)}\right) \quad \forall p_{(n)} \in \mathbb{N} \mid p_{(n)} \geq 13
$$

Necessarily therefore, we have a contradiction to the initial hypothesis. Since at the twin primes the sequence $\exp \left(p_{(n)}\right)-p_{(n)} \sharp \exp \left(\mathcal{L B}_{p_{(n)}}\right)$ approaches:

$$
\left[\exp \left(p_{(n)}\right)-p_{(n)} \sharp \exp \left(\mathcal{L B}_{p_{(n)}}\right)\right] \sim \exp (2)\left[\exp \left(p_{(n-1)}\right)-p_{(n-1)} \sharp \exp \left(\mathcal{L B}_{p_{(n-1)}}\right)\right]
$$

Rearranging the above we obtain that, at the twin primes the primorial approaches: (4.30)

$$
p_{(n)} \sharp \exp \left(\mathcal{L B}_{p_{(n)}}\right) \sim \exp \left(p_{(n)}\right)-\exp (2)\left[\exp \left(p_{(n-1)}\right)-p_{(n-1)} \sharp \exp \left(\mathcal{L B}_{p_{(n-1)}}\right)\right]
$$

This in turn implies that a strict inequality holds:

$$
p_{(n)} \sharp \exp \left(\mathcal{L B}_{p_{(n)}}\right)<\exp \left(p_{(n)}\right)
$$

Since increasing the gap between the consecutive primes has the effect of exponentially increasing the value that the sequence $a_{(n)}$ attains, therefore this result holds for all $p_{(n)} \in \mathbb{N} \mid p_{(n)} \geq 13$. By taking the logarithms, we obtain:

$$
\mathcal{L B}_{p_{(n)}}<\left(p_{(n)}-\log p_{(n)} \sharp\right) \quad \forall p_{(n)} \in \mathbb{N} \mid p_{(n)} \geq 13
$$

Thus, Lemma 4.5 holds for all $p_{(n)} \in \mathbb{N} \mid p_{(n)} \geq 13$. Direct computation verifies that Lemma 4.5 holds for all $p_{(n)} \in \mathbb{N} \mid 2 \leq p_{(n)} \leq 13$. Therefore, Lemma 4.5 holds as stated, concluding the proof.

Lemma 4.7 (Upper Estimation Error Bound On The Difference $p_{n}-\log p_{n} \sharp$ ). The error of estimation of the primorial function by the use of the value of $p_{(n)}$ imposes the following upper bound:

$$
\left(p_{(n)}-\log p_{(n)} \sharp\right)<2 \sqrt{p_{(n)}}=\mathcal{U} \mathcal{B}_{p_{(n)}} \quad \forall p_{(n)} \in \mathbb{N} \mid p_{(n)} \geq 2
$$

where $\gamma \approx 0.57721566490153286060651209$ is the Euler-Mascheroni constant. 
Proof.

Both $\exp \left(p_{(n)}\right)$ and $p_{(n)} \sharp$ as well as $\exp \left(\mathcal{U} \mathcal{B}_{p_{(n)}}\right)$ are monotone, divergent sequences of positive real numbers for all $p_{(n)} \in \mathbb{N} \mid p_{(n)} \geq 2$. Suppose that Lemma 4.7 is false. From inequality 4.33, in accordance with the hypothesis we derive:

$$
\left(p_{(n)} \sharp\right) \exp \left(\mathcal{U B}_{p_{(n)}}\right)-\exp \left(p_{(n)}\right)<0
$$

However, at $p_{n}=13$ the difference 4.34 attains $\sim 4.02297598 \times 10^{7}$ and further diverges exponentially. Therefore we apply the d'Alemberts Ratio Test. Define a sequence for all prime numbers $p_{(n-1)}, p_{(n)} \in \mathbb{N} \mid p_{(n)} \geq 13$ :

Definition 4.8. $a_{(n)}=\frac{\left(p_{(n)} \sharp\right) \exp \left(\mathcal{U} \mathcal{B}_{p_{(n)}}\right)-\exp \left(p_{(n)}\right)}{\left(p_{(n-1)} \sharp\right) \exp \left(\mathcal{U} \mathcal{B}_{p_{(n-1)}}\right)-\exp \left(p_{(n-1)}\right)} \quad \forall p_{(n)} \in \mathbb{N} \mid p_{(n)} \geq 13$

Remark 4.4. The terms of the sequence $a_{(n)}$ given by the Definition 4.8 have the least value at the twin primes since the difference $p_{(n)}-p_{(n-1)}=2$. Consequently, it is both necessary and sufficient, to consider the sequence 4.8 at the twin primes only, with $p_{(n)}=6 i+7 \mid i \in \mathbb{N}, i \geq 1$.

At the twin primes:

$$
\exp \left(p_{(n)}\right)=\exp \left(p_{(n-1)}+2\right)=\exp \left(p_{(n-1)}\right) \times \exp (2)
$$

Further,

$$
p_{(n)} \sharp=p_{(n-1)} \sharp\left(p_{(n)}\right)
$$

Thus, at the twin primes the sequence $a_{(n)}$ equals:

$$
a_{(n)}=p_{(n)} \times\left[\frac{\left(p_{(n-1)} \sharp\right) \exp \left(\mathcal{U B}_{p_{(n)}}\right)-\frac{\exp \left(p_{(n)}\right)}{\left(p_{(n)}\right)}}{\left(p_{(n-1)} \sharp\right) \exp \left(\mathcal{U} \mathcal{B}_{p_{(n-1)}}\right)-\exp \left(p_{(n-1)}\right)}\right]
$$

The bracketed expression on the RHS, at the twin primes approaches the limit:

$$
\lim _{n \rightarrow \infty}\left[\frac{\left(p_{(n-1)} \sharp\right) \exp \left(\mathcal{U B}_{p_{(n)}}\right)-\frac{\exp \left(p_{(n)}\right)}{\left(p_{(n)}\right)}}{\left(p_{(n-1)} \sharp\right) \exp \left(\mathcal{U} \mathcal{B}_{p_{(n-1)}}\right)-\exp \left(p_{(n-1)}\right)}\right] \rightarrow 1
$$

at the twin primes therefore, the sequence $a_{(n)}$ must clearly approach the limit:

$$
\begin{aligned}
& \lim _{n \rightarrow \infty} a_{(n)} \\
& \quad=\lim _{n \rightarrow \infty}\left[\left[\frac{\left(p_{(n-1)} \sharp\right) \exp \left(\mathcal{U} \mathcal{B}_{p_{(n)}}\right)-\frac{\exp \left(p_{(n)}\right)}{\left(p_{(n)}\right)}}{\left(p_{(n-1)} \sharp\right) \exp \left(\mathcal{U} \mathcal{B}_{p_{(n-1)}}\right)-\exp \left(p_{(n-1)}\right)}\right] p_{(n)}\right] \rightarrow p_{(n)}
\end{aligned}
$$

By the d'Alemberts Ratio Test, the series formed from the terms of the difference $\left(p_{(n)} \sharp\right) \exp \left(\mathcal{U B}_{p_{(n)}}\right)-\exp \left(p_{(n)}\right)$ diverges, as $p_{(n)}$ increases unboundedly. Thus, it logically follows that:

$$
p_{(n)} \sharp \exp \left(\mathcal{U} \mathcal{B}_{p_{(n)}}\right) \geq \exp \left(p_{(n)}\right) \quad \forall p_{(n)} \in \mathbb{N} \mid p_{(n)} \geq 13
$$

Necessarily therefore, we have a contradiction to the initial hypothesis. Since at the twin primes the sequence $\left(p_{(n)} \sharp\right) \exp \left(\mathcal{U B}_{p_{(n)}}\right)-\exp \left(p_{(n)}\right)$ approaches: (4.41) $\left[\left(p_{(n)} \sharp\right) \exp \left(\mathcal{U B}_{p_{(n)}}\right)-\exp \left(p_{(n)}\right)\right] \sim\left(p_{(n)}\right)\left[\left(p_{(n-1)} \sharp\right) \exp \left(\mathcal{U} \mathcal{B}_{p_{(n-1)}}\right)-\exp \left(p_{(n-1)}\right)\right]$ 
Rearranging the above we obtain, that at the twin primes the primorial approaches: $(4.42)$

$p_{(n)} \sharp \exp \left(\mathcal{U B}_{p_{(n)}}\right) \sim \exp \left(p_{(n)}\right)+\left(p_{(n)}\right)\left[\left(p_{(n-1)} \sharp\right) \exp \left(\mathcal{U} \mathcal{B}_{p_{(n-1)}}\right)-\exp \left(p_{(n-1)}\right)\right]$

This in turn implies that a strict inequality holds:

$$
p_{(n)} \sharp \exp \left(\mathcal{U B}_{p_{(n)}}\right)>\exp \left(p_{(n)}\right)
$$

Since increasing the gap between the consecutive primes has the effect of exponentially increasing the value that the sequence $a_{(n)}$ attains, therefore this result holds for all $p_{(n)} \in \mathbb{N} \mid p_{(n)} \geq 13$. By taking the logarithms, we obtain:

$$
\mathcal{U B}_{p_{(n)}}>\left(p_{(n)}-\log p_{(n)} \sharp\right) \quad \forall p_{(n)} \in \mathbb{N} \mid p_{(n)} \geq 13
$$

Thus, Lemma 4.7 holds for all $p_{(n)} \in \mathbb{N} \mid p_{(n)} \geq 13$. Direct computation verifies that Lemma 4.7 holds for all $p_{(n)} \in \mathbb{N} \mid 2 \leq p_{(n)} \leq 13$. Therefore, Lemma 4.7 holds as stated, concluding the proof. 
5. The prime counting function $\pi_{(n)}$ Supremum/Infimum Bounds

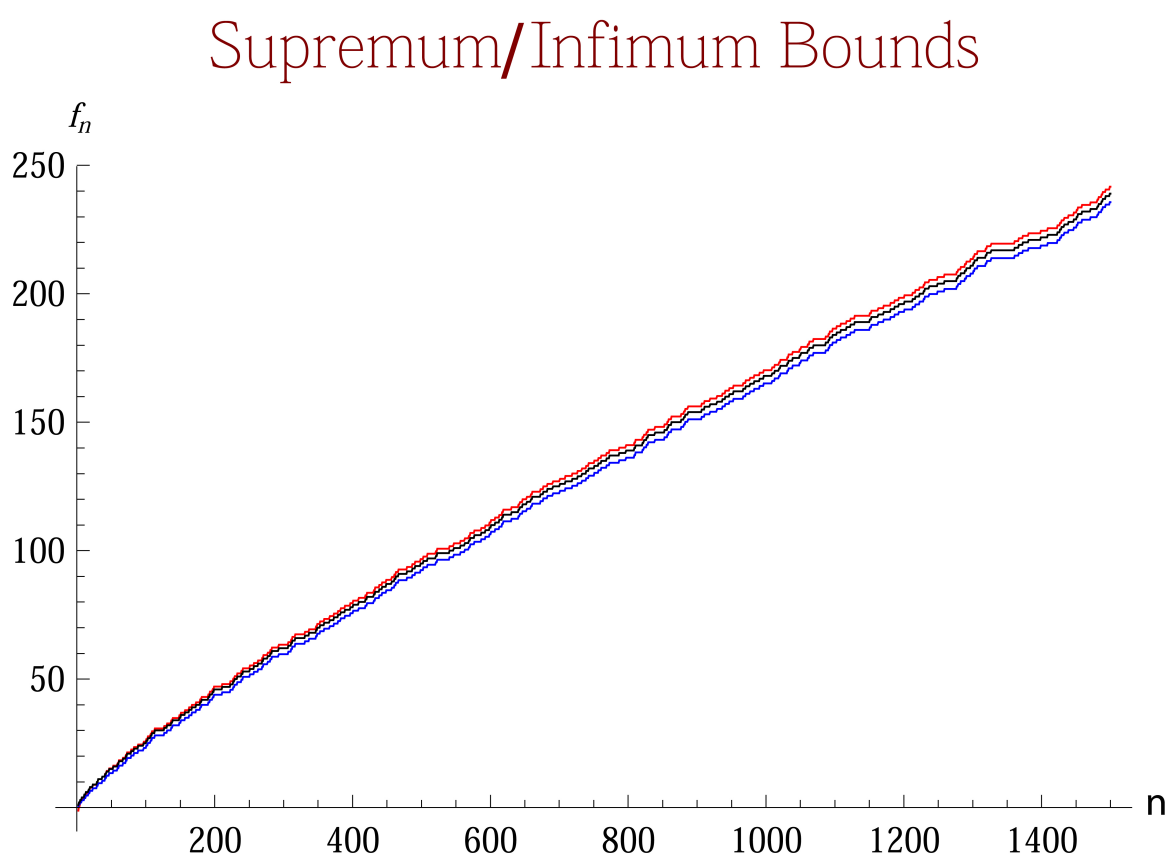

FiguRE 2. The drawing shows the Supremum (Red) and Infimum (Blue) bounds on $\pi_{n}$ (Black).
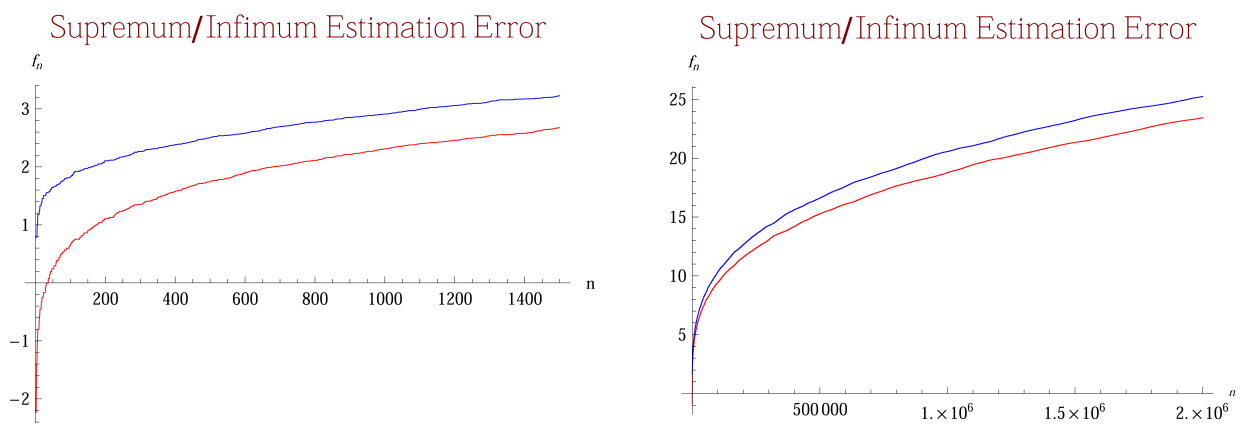

Figure 3. The drawing shows the estimation error made by the Supremum (Red) and Infimum (Blue) of the prime counting function $\pi_{n}$. The figures are drawn at every $n \in \mathbb{N}$ in the range. 


\subsection{The Tailored logarithmic integral definitions.}

Definition 5.1 (Supremum Upper integration limit).

$$
\theta_{\mathcal{S}}=\log p_{n} \sharp=\sum_{p \leq n} \log p \quad \text { where } p \text { is the prime } p \in \mathbb{N} \mid p \leq n
$$

Definition 5.2 (Infimum Upper integration limit).

$$
\theta_{\mathcal{I}}=\sum_{p \leq n}\left((\log (p))-\frac{\pi(\log \log p)}{(p)^{\gamma}}\right) \text { where } p \text { is the prime } p \in \mathbb{N} \mid p \leq n
$$

and $\gamma$ is the Euler-Mascheroni constant $\gamma \approx 0.57721566490153286060651209$.

Theorem 5.3 (Supremum and Infimum Bounds on $\pi_{(n)}$ ).

Let $\pi_{n}$ denote the prime counting function. Then, the Supremum and Infimum bounds, which constitute bounds on $\pi_{n}$ are given by:

$$
\begin{gathered}
\operatorname{Inf}_{n}=\int_{2}^{\theta_{\mathcal{I}}} \frac{d t}{\log t}<\pi_{n} \quad \forall n \in \mathbb{N} \mid n \geq 11 \\
\pi_{n}<\int_{2}^{\theta_{\mathcal{S}}} \frac{d t}{\log t}=\operatorname{Sup}_{n}=T L i_{(n)} \quad \forall n \in \mathbb{N} \mid n \geq 43
\end{gathered}
$$

\section{Remark 5.1.}

The proof is a multistep regimen and will be carried over several of the following theorems/lemmas. The proof pertains to the Supremum Bound only, an analogous process needs to be carried out in the case of the Infimum Bound, which is left at the discretion of the reader.

Graphs in this section implement a variant of logarithmic scaling of the horizontal axis given by:

Definition 5.4 (Scaling factor). $\xi=\frac{\log _{10}\left(\frac{n}{24}\right)}{\log _{10}(24)}$

\subsection{Preliminary theory.}

Tailored logarithmic integral Supremum/Infimum bounds, as given by Theorem 5.3 , present a significantly improved accuracy of estimation of the function $\pi_{(n)}$. Lemma 4.3 states:

Consequently,

$$
\log p_{(n)} \sharp<p_{(n)} \leq n \quad \forall n \in \mathbb{N} \mid n \geq 2
$$

$$
\frac{\log p_{(n)} \sharp}{\log n}<\frac{n}{\log n} \quad \forall n \in \mathbb{N} \mid n \geq 2
$$

Remark 5.2. The classical offset logarithmic integral $L i_{(n)}$ of C.F. Gauss, is an improvement of the estimate of the number of primes given by $n / \log n$, up to some $n \in \mathbb{N}$. Therefore, since the left side of the inequality 5.3 increases only at the primes as $\pi_{n}$ does, it constitutes an improvement in $\pi_{(n)}$ estimation. Numerical 
Estimation Error Comparison: Gauss' vs Tailored Integral FunctionValue

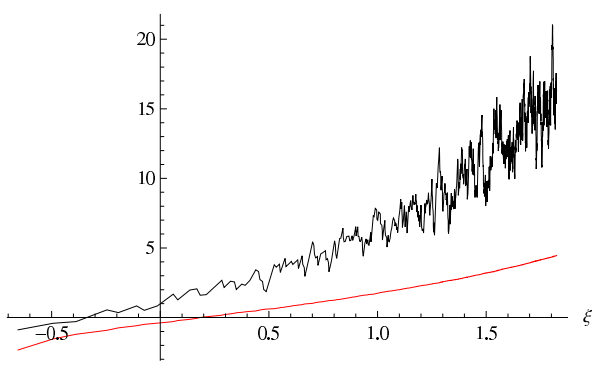

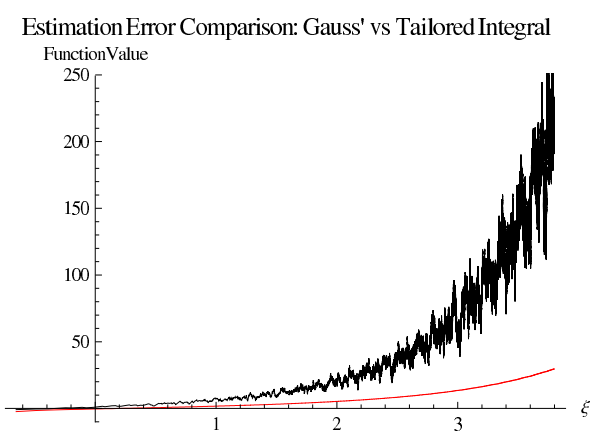

FIGURE 4. The drawings contrast the difference in estimation error made by the Gauss' Logarithmic Integral (black) vs the Tailored Integral (red). The figures drawn with respect to $\xi$, give the range up to $n=7919$ (left), and up to $n=4256233$ (right).

comparison of the performance of the Carl F. Gauss offset $L i_{(n)}$ vs the $T L i_{(n)}$ is given in Table 2, and graphically presented in Fig. 4.

The graph of the tailored integral is below that of $\pi_{(n)}$ for all $n \in \mathbb{N} \mid n<$ 43, please refer to Fig. 6a. Since the primorial function increases only at the primes, necessarily therefore, the estimation error of the tailored integral increases at the primes only. Hence, if the relation $T L i_{(n)} \geq \pi_{(n)}$ holds at the primes, it therefore holds at every other point. This contrasts strongly with the Gauss' logarithmic integral $L i_{(n)}$ in which, the estimation error term increases over the intervals between the primes and decreases at the primes. As a result, it produces large estimation error oscillations. On the other hand $T L i_{(n)}$, accurately duplicates the pattern of the curve of $\pi_{(n)}$, with minimal error increase.

TABle 2. Comparison: Gauss' $L i_{(n)}$ vs $T L i_{(n)}$

\begin{tabular}{|l|l|l|l|l|l|}
\hline$n$ & $\pi_{(n)}$ & $L i_{(n)}$ & $L i_{(n)}-\pi_{(n)}$ & $T L i_{(n)}$ & $T L i_{(n)}-\pi_{(n)}$ \\
\hline 47 & 15 & 16.6506001 & 1.6506001 & 15.0538449 & 0.0538449 \\
\hline 97 & 25 & 28.427383 & 3.42738 & 25.4798052 & 0.4798052 \\
\hline 997 & 168 & 176.1301053 & 8.130105 & 170.2098996 & 2.2098996 \\
\hline 9973 & 1229 & 1242.160134 & 13.160134 & 1233.7930553 & 4.7930553 \\
\hline 99991 & 9592 & 9627.982104 & 35.982104 & 9601.4333493 & 9.4333493 \\
\hline 999983 & 78498 & 78625.273494 & 127.273494 & 78516.778831 & 18.7788305 \\
\hline 9999991 & 664579 & 664916.801506 & 337.801506 & 664618.2703 & 39.27032 \\
\hline 99999989 & 5761455 & 5762207.73313 & 752.733129 & 5761542.23 & 87.22988 \\
\hline 999999937 & 50847534 & 50849230.8718 & 1696.87178 & 50847736.926 & 202.925523 \\
\hline 9999999967 & 455052511 & 455055612.108 & 3101.10829 & 455052999.99 & 488.985131 \\
\hline 99999999977 & 4118054813 & 4118066398.67 & 11585.6684 & 4118056037.8 & 1224.81062 \\
\hline
\end{tabular}

\subsection{Tailored integral $T L i_{(n)}$ step sequence.}

Due to the fact that $T L i_{(n)}$ increases stepwise at the primes, the analysis of the step size and its limit as $n$ approaches infinity forms the core of the proof of the 

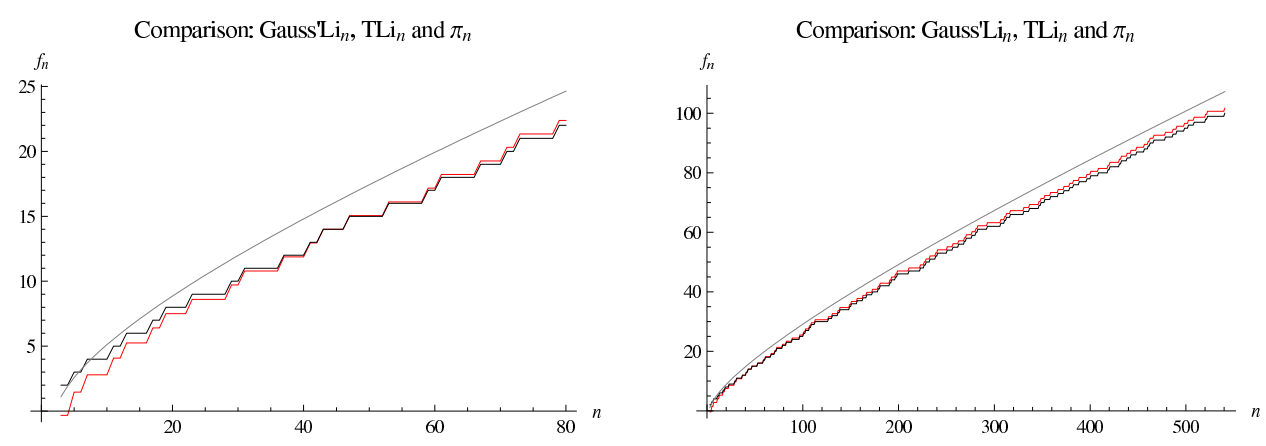

Figure 5. The figures drawn at every $n \in \mathbb{N}$ in the range, show the graphs of $L i_{(n)}$ (grey), $\pi_{(n)}$ (black) and $T L i_{(n)}$ (red).
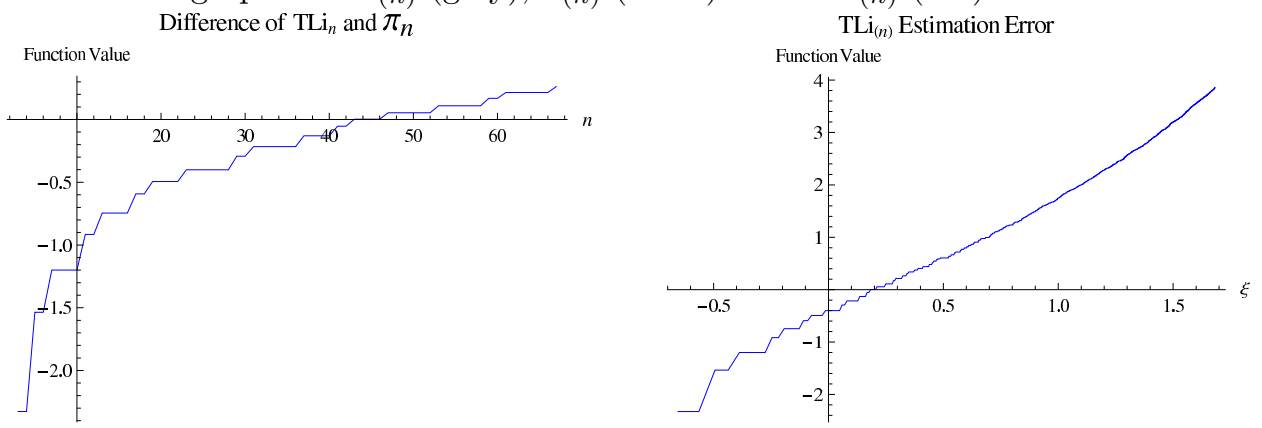

Figure 6 . The drawings show the estimation error $T L i_{(n)}-\pi_{(n)}$ curve drawn at every $n \in \mathbb{N}$ within the pertinent range. The right figure drawn with respect to $\xi$, corresponding to: $3 \leq n \leq 5,000$.

tailored integral. Thus, for a pair of consecutive primes: $p_{i}$ and $p_{i+1}$ we define the stepwise limits of integration for all $p_{i} \in \mathbb{N} \mid p_{i} \geq 2$ :

Definition 5.5 (Stepwise lower integration limit). $\theta_{1}=\log p_{(i)} \sharp$

Definition 5.6 (Stepwise upper integration limit). $\theta_{2}=\log p_{(i+1)} \sharp$

Definition 5.7 (Step sequence).

$$
\mathcal{S S} \mathcal{Q}_{\left(p_{(i+1)}\right)}=\left(T L i_{\left(p_{(i+1)}\right)}-T L i_{\left(p_{i}\right)}\right)=\int_{\theta_{1}}^{\theta_{2}} \frac{d t}{\log t} \quad \forall p_{i} \in \mathbb{N} \mid p_{i} \geq 3
$$

Definition 5.8 (Step estimation error sequence).

$$
\mathcal{S E R}_{\left(p_{(i+1)}\right)}=\left(T L i_{\left(p_{(i+1)}\right)}-T L i_{\left(p_{i}\right)}-1\right)=\int_{\theta_{1}}^{\theta_{2}} \frac{d t}{\log t}-1 \quad \forall p_{i} \in \mathbb{N} \mid p_{i} \geq 3
$$

Remark 5.3. Both the tailored integral $T L i_{(n)}$ and $\pi_{n}$ are weakly monotone functions, increasing unboundedly, hence producing a positive sequence of numbers which diverges to infinity. The initial estimates of the $T L i_{(n)}$ step size indicate that the step sequence quickly approaches the value of 1 from above. Table 3 presents some of the values that the step sequence takes at the powers of 10 . 
It is obvious that the numerical value attained by the step sequence at various points fluctuates as well, as a consequence of the size of the gap between the two consecutive primes (as well as the distance to the preceding prime pair). The effect however, of the gap interval length rapidly decreases as $p_{i}$ increases, because the prime gaps are bounded above (Theorems 3.6 and 3.7).

Lemma 5.9 (Stepwise Convergence Of The Error of Estimation of the $\left.T L i_{(n)}\right)$. The step sequence of the tailored logarithmic integral $T L i_{(n)}$ is Cauchy and converges asymptotically from above to the limit:

$$
\lim _{p_{i} \rightarrow \infty}\left(\int_{\theta_{1}}^{\theta_{2}} \frac{d t}{\log t}\right)=1
$$

Furthermore, the difference of the step integral $T L i_{(n)}$ and its approximation has the following bounds:

$$
\begin{array}{r}
L D B_{p_{(i+1)}}=\frac{1}{5\left(p_{(i+1)}\right)} \leq\left[\int_{\theta_{1}}^{\theta_{2}} \frac{d t}{\log t}-\frac{\log p_{(i+1)}}{\log \left(\log p_{(i+1)} \sharp\right)}\right] \leq \frac{1}{p_{(i+1)}}=U D B_{p_{(i+1)}} \\
\text { for all } p \in \mathbb{N} \mid p \geq 13
\end{array}
$$

with $\theta_{1}$ and $\theta_{2}$ given by the Definitions 5.5 and 5.6 respectively.

Proof.

By the Prime Number Theorem we may estimate the integral $T L i_{(n)}$ step sequence for any prime number $p \in \mathbb{N} \mid p \geq 3$ :

$$
\int_{\theta_{1}}^{\theta_{2}} \frac{d t}{\log t} \sim \frac{\theta_{2}-\theta_{1}}{\log \theta_{2}}=\frac{\left(\log p_{(i)} \sharp+\log p_{(i+1)}\right)-\log p_{(i) \sharp}}{\log \left(\log p_{(i+1)} \sharp\right)}=\frac{\log p_{(i+1)}}{\log \left(\log p_{(i+1)} \sharp\right)}
$$

Thus by the PNT we have,

$$
\int_{\theta_{1}}^{\theta_{2}} \frac{d t}{\log t} \sim \frac{\log p_{(i+1)}}{\log \left(\log p_{(i+1)} \sharp\right)}
$$

The logarithm of the primorial function is clearly a monotone function increasing unboundedly, hence, producing a sequence of positive real numbers which diverges to infinity. From Lemma 4.3 we have that $\log p_{(i+1)} \sharp$ is asymptotic from below to $p_{(i+1)}$, as well as:

$$
\begin{aligned}
\log p_{(i+1)} \sharp<p_{(i+1)} \leq n \quad \forall n & \in \mathbb{N} \mid n \geq 2, \\
& \text { where } p_{(i+1)} \text { is the greatest prime } p \in \mathbb{N} \mid p \leq n
\end{aligned}
$$

Hence, for a prime number $p \in \mathbb{N}$,

$$
\log \left(\log p_{(i+1)} \sharp\right) \leq \log p_{(i+1)}
$$

This implies that the estimating sequence converges asymptotically from above to the limit:

$$
\lim _{p_{(i+1)} \rightarrow \infty}\left(\frac{\log p_{(i+1)}}{\log \left(\log p_{(i+1)} \sharp\right)}\right) \rightarrow 1
$$


Therefore it is Cauchy. The step integral $T L i_{(n)}$ at $p_{6}=13$ attains $\sim 1.13056$ and the step sequence values decrease, asymptotically approaching 1 as $p_{n}$ increases unboundedly. Please, also refer to the Table 3. Consequently,

$$
\lim _{p_{(i+1)} \rightarrow \infty}\left(\int_{\theta_{1}}^{\theta_{2}} \frac{d t}{\log t}\right)=\lim _{p_{(i+1)} \rightarrow \infty}\left(\frac{\log p_{(i+1)}}{\log \left(\log p_{(i+1)} \sharp\right)}\right) \rightarrow 1
$$

Thus the step integral $T L i_{(n)}$ is Cauchy as well. Both $L D B_{p_{(i+1)}}$ and $U D B_{p_{(i+1)}}$ are clearly strictly monotone decreasing Cauchy sequences. Suppose that the following assertion is false:

$$
\frac{1}{5\left(p_{(i+1)}\right)} \leq\left[\int_{\theta_{1}}^{\theta_{2}} \frac{d t}{\log t}-\frac{\log p_{(i+1)}}{\log \left(\log p_{(i+1)} \sharp\right)}\right]
$$

This implies that:

$$
5\left(p_{(i+1)}\right)-\left[1 /\left(\int_{\theta_{1}}^{\theta_{2}} \frac{d t}{\log t}-\frac{\log p_{(i+1)}}{\log \left(\log p_{(i+1)} \sharp\right)}\right)\right]<0
$$

However, at $p_{(n)}=13$ the inequality 5.11 attains $\sim 48.6109$ and diverges as $p_{(n)}$ increases unboundedly with the rate of divergence $\propto k p_{(n)}$ s.t. $k \sim 3$ for larger primes $p_{(n)}$. Consequently, we have a contradiction to the hypothesis. Inequality 5.10 therefore, is valid for all $p_{n} \in \mathbb{N} \mid p_{n} \geq 13$.

Suppose now, that the following inequality is false:

$$
\left[\int_{\theta_{1}}^{\theta_{2}} \frac{d t}{\log t}-\frac{\log p_{(i+1)}}{\log \left(\log p_{(i+1)} \sharp\right)}\right] \leq \frac{1}{p_{(i+1)}}
$$

This implies that:

$$
\left[1 /\left(\int_{\theta_{1}}^{\theta_{2}} \frac{d t}{\log t}-\frac{\log p_{(i+1)}}{\log \left(\log p_{(i+1)} \sharp\right)}\right)\right]-p_{(i+1)}<0
$$

However, at $p_{(n)}=13$ the inequality 5.13 attains $\sim 3.38914$ and diverges as $p_{(n)}$ increases unboundedly with the rate of divergence $\propto k p_{(n)}$ s.t. $k \sim 1$ for larger primes $p_{(n)}$. Consequently, we have a contradiction to the hypothesis. Inequality 5.12 therefore, is valid for all $p_{n} \in \mathbb{N} \mid p_{n} \geq 13$. Necessarily this implies that the Inequality 5.5 holds as stated. This demonstrates therefore, that since $U D B_{p_{(i+1)}}$ is strictly monotone decreasing Cauchy sequence with a limit $\mathcal{L}=0$ :

$$
\lim _{\left(p_{(i+1)}\right) \rightarrow \infty}\left(\frac{1}{p_{(i+1)}}\right)=\lim _{\left(p_{(i+1)}\right) \rightarrow \infty}\left[\int_{\theta_{1}}^{\theta_{2}} \frac{d t}{\log t}-\frac{\log p_{(i+1)}}{\log \left(\log p_{(i+1)} \sharp\right)}\right] \rightarrow 0
$$

Thus, from above we have that the estimating sequence 5.8 converges asymptotically from above to its limit $\mathcal{L}=1$. Since the step integral at $p_{(i+1)}=11$ attains $\sim 1.2171$ necessarily therefore the step integral tends asymptotically from above:

$$
\lim _{p_{(i+1)} \rightarrow \infty}\left(\frac{\log p_{(i+1)}}{\log \left(\log p_{(i+1)} \sharp\right)}\right)=\lim _{p_{(i+1)} \rightarrow \infty}\left(\int_{\theta_{1}}^{\theta_{2}} \frac{d t}{\log t}\right) \rightarrow 1
$$


This implies, that the sequence of the step estimation errors asymptotically converges from above (also refer to Table 3 ):

$$
\lim _{p_{(i+1)} \rightarrow \infty}\left(\left(\int_{\theta_{1}}^{\theta_{2}} \frac{d t}{\log t}\right)-1\right)=\lim _{p_{(i+1)} \rightarrow \infty}\left(\frac{\log p_{(i+1)}}{\log \left(\log p_{(i+1)} \sharp\right)}-1\right) \rightarrow 0
$$

Thus concluding the proof of Lemma 5.9.

TABLE 3. Step sequence values

\begin{tabular}{|c|c|l|l|l|}
\hline$p_{i}$ & $p_{i+1}$ & Actual step & Est. step Eq. 5.6 & Difference \\
\hline 7 & 11 & 1.284296315549 & 1.17139190927 & 0.112904406279 \\
\hline 97 & 101 & 1.036036760682 & 1.029877441266 & 0.006159319416 \\
\hline 997 & 1009 & 1.007295189211 & 1.006767382731 & 0.000527806480 \\
\hline 9973 & 10007 & 1.001161895309 & 1.001111285430 & 0.000050609879 \\
\hline 99991 & 100003 & 1.000271361223 & 1.000266343124 & $5.0180995783 \times 10^{-6}$ \\
\hline 999983 & 1000003 & 1.000109530674 & 1.00010902981 & $5.00863978704 \times 10^{-7}$ \\
\hline 9999991 & 10000019 & 1.000029984472 & 1.000029934445 & $5.0027059524 \times 10^{-8}$ \\
\hline 99999989 & 100000007 & 1.000006660234 & 1.000006655234 & $5.00067955427 \times 10^{-9}$ \\
\hline
\end{tabular}

Remark 5.4. The integral part of the step size clearly accounts for the prime number found. Comparing each fractional part of the step (please refer to Table 3) at $p_{i}$ with the corresponding term of the harmonic series $\left(\frac{1}{p_{i}}\right)$, it becomes obvious that it is greater than the term of the series. Since the error of estimation is the sum of $\pi_{(n)}$ of such individual terms, comparing its sum with the divergent sum of reciprocals of successive prime numbers leads to a conjecture, that the sum of the estimation error terms diverges as $p_{i}$ tends to infinity.

\subsection{Step sequence estimation error bounds.}

Corollary 5.10 (Infimum and Supremum Step Sequence Estimation Error Bounds). The step sequence error of estimation of the prime counting function $\pi_{(n)}$ by the application of the tailored logarithmic integral $T L i_{(n)} \forall p_{(i)} \in \mathbb{N} \mid p_{(i)} \geq 13$, is bounded below/above by:

$$
\begin{aligned}
& \mathcal{I N} \mathcal{F}_{p_{(i+1)}}=\frac{\log p_{(i+1)}}{\log \left(\log p_{(i+1)} \sharp\right)}+\frac{1}{5\left(p_{(i+1)}\right)}-1 \leq\left[\int_{\theta_{1}}^{\theta_{2}} \frac{d t}{\log t}-1\right] \\
& \quad \leq \frac{\log p_{(i+1)}}{\log \left(\log p_{(i+1)} \sharp\right)}+\frac{1}{p_{(i+1)}}-1=\mathcal{S U} \mathcal{P}_{p_{(i+1)}} \quad \text { for all } p \in \mathbb{N} \mid p \geq 13
\end{aligned}
$$

where $p_{(i)}$ and $p_{(i+1)}$ are associated with lower/upper limits of integration and $\theta_{1}$, $\theta_{2}$ are given by the Definitions 5.5 and 5.6 respectively.

Proof. From Lemma 5.9 we have:

$$
\frac{1}{5\left(p_{(i+1)}\right)} \leq\left[\int_{\theta_{1}}^{\theta_{2}} \frac{d t}{\log t}-\frac{\log p_{(i+1)}}{\log \left(\log p_{(i+1)} \sharp\right)}\right] \leq \frac{1}{p_{(i+1)}} \quad \text { for all } p \in \mathbb{N} \mid p \geq 13
$$


Which is equivalent to say that:

$$
\begin{aligned}
\frac{\log p_{(i+1)}}{\log \left(\log p_{(i+1)} \sharp\right)}+ & \frac{1}{5\left(p_{(i+1)}\right)}-1 \leq\left[\int_{\theta_{1}}^{\theta_{2}} \frac{d t}{\log t}-1\right] \\
& \leq \frac{\log p_{(i+1)}}{\log \left(\log p_{(i+1)} \sharp\right)}+\frac{1}{p_{(i+1)}}-1 \quad \text { for all } p \in \mathbb{N} \mid p \geq 13
\end{aligned}
$$

thus completing the proof.

The Infimum and Supremum error bounds $\mathcal{I} \mathcal{S} \mathcal{E}_{\left(p_{(n)}\right)}$ and $\mathcal{S} \mathcal{S E}\left(\mathcal{E}_{\left(p_{(n)}\right)}\right.$ for the tailored integral step estimation error are computationally very demanding. Therefore, Theorems: 5.11 and 5.12 that follow, establish simpler bounds.

Theorem 5.11 (The Step Sequence Estimation Error Lower Bound).

The estimation error of the tailored logarithmic integral $T L i_{(n)}$ at every step exceeds the value of the inverse of the pertinent prime number hence, it is bounded below by $1 / p \forall p \in \mathbb{N} \mid p_{i} \geq 13$ :

$$
\operatorname{SER}_{\left(p_{(i+1)}\right)}=\left(\int_{\theta_{1}}^{\theta_{2}} \frac{d t}{\log t}\right)-1>\frac{1}{p_{(i+1)}}
$$

where $p_{(i)}$ and $p_{(i+1)}$ are associated with the lower/upper limit of integration $\theta_{1}$ and $\theta_{2}$ respectively.

Proof.

By Lemma 5.9 the sequence $\mathcal{S E \mathcal { R }}\left(p_{(i+1)}\right)$ is Cauchy and it converges from above to the limit $\mathcal{L}=0$. The sequence of the reciprocals of prime numbers is clearly Cauchy and converges to the limit $\mathcal{L}=0$. By Lemma 5.9 we have that:

$$
\frac{1}{5\left(p_{(i+1)}\right)}+\frac{\log p_{(i+1)}}{\log \left(\log p_{(i+1)} \sharp\right)}-1 \leq \int_{\theta_{1}}^{\theta_{2}} \frac{d t}{\log t}-1 \quad \text { for all } p \in \mathbb{N} \mid p \geq 13
$$

Consequently Theorem 5.11 is valid if and only if:

$$
\frac{1}{p_{(i+1)}} \leq \frac{1}{5\left(p_{(i+1)}\right)}+\frac{\log p_{(i+1)}}{\log \left(\log p_{(i+1)} \sharp\right)}-1
$$

Now,

$$
\begin{aligned}
\frac{1}{5\left(p_{(i+1)}\right)}+\frac{\log p_{(i+1)}}{\log \left(\log p_{(i+1)} \sharp\right)}-1-\frac{1}{p_{(i+1)}} & \\
= & \frac{\left(5\left(p_{(i+1)}\right)\left(\log \left(p_{(i+1)}\right)\right)\right)-\left(4+5\left(p_{(i+1)}\right)\right)\left(\log \left(\log \left(p_{(i+1)} \sharp\right)\right)\right)}{\left(5\left(p_{(i+1)}\right)\right)\left(\log \left(\log \left(p_{(i+1)} \sharp\right)\right)\right)}
\end{aligned}
$$

From Lemma 4.3 we have that $\log p_{(i+1)} \sharp$ is asymptotic (from below):

$$
\log p_{(i+1)} \sharp \sim p_{(i+1)}
$$

as well as:

$$
\log p_{(i+1)} \sharp<p_{(i+1)} \leq n \quad \forall n \in \mathbb{N} \mid n \geq 2
$$

Hence,

$$
\log \left(\log p_{(i+1)} \sharp\right) \leq \log p_{(i+1)}
$$


From Lemma 4.5 we have for all $p_{(i+1)} \in \mathbb{N} \mid p_{(i+1)} \geq 2$ :

$$
\mathcal{L B}_{p_{(i+1)}}=(\sqrt{5}-1)\left(4 \gamma^{2}-2 \gamma\right)\left(\log p_{(i+1)}\right) \sqrt[3]{p_{(i+1)}}<\left(p_{(i+1)}-\log p_{(i+1)} \sharp\right)
$$

Consequently, from the above we obtain:

$$
\left(p_{(i+1)}-\mathcal{L B}_{p_{(i+1)}}>\log p_{(i+1)} \sharp\right)
$$

Bearing in mind that for all positive $a, b \in \mathbb{R} \mid a>b$ :

$$
\log (a+b)=\log (a(1+b / a))=\log (a)+\log \left(1+\frac{b}{a}\right)
$$

Thus, by Lemma 4.3 we have:

$$
5 p_{(i+1)}\left(\log \left(p_{(i+1)}-\mathcal{L B}_{p_{(i+1)}}\right)\right) \geq 5 p_{(i+1)}\left(\log \left(\log \left(p_{(i+1)} \sharp\right)\right)\right)
$$

Hence,

$$
\begin{aligned}
& 5 p_{(i+1)}\left(\log \left(p_{(i+1)}\right)\right)=5 p_{(i+1)}\left(\log \left(p_{(i+1)}-\mathcal{L B}_{p_{(i+1)}}+\mathcal{L} \mathcal{B}_{p_{(i+1)}}\right)\right) \\
& =5 p_{(i+1)}\left[\log \left(p_{(i+1)}-\mathcal{L B}_{p_{(i+1)}}\right)+\log \left(1+\frac{\mathcal{L B}_{p_{(i+1)}}}{\left(p_{(i+1)}-\mathcal{L} \mathcal{B}_{p_{(i+1)}}\right)}\right)\right] \\
& \geq 5 p_{(i+1)}\left(\log \left(\log \left(p_{(i+1)} \sharp\right)\right)\right)
\end{aligned}
$$

Suppose that the Theorem 5.11 is false. Then it must be true that the numerator of equation 5.21 is less than zero. From inequality 5.26 we see that without loss of generality, upon substitution into the numerator of the inequality 5.21, we can drop the common terms obtaining:

$$
\begin{aligned}
& \left(5\left(p_{(i+1)}\right)\left(\log \left(p_{(i+1)}\right)\right)\right)-\left(4+5\left(p_{(i+1)}\right)\right)\left(\log \left(\log \left(p_{(i+1)} \sharp\right)\right)\right) \\
& \geq 5 p_{(i+1)}\left[\log \left(1+\frac{\mathcal{L B}_{p_{(i+1)}}}{\left(p_{(i+1)}-\mathcal{L} \mathcal{B}_{p_{(i+1)}}\right)}\right)\right]-4\left(\log \left(\log \left(p_{(i+1)} \sharp\right)\right)\right)<0
\end{aligned}
$$

However at $p_{(i+1)}=37$ the difference 5.27 attains $\sim 0.20084385349345676$ and diverges. Hence we have a contradiction to our hypothesis which implies that the inequality is true:

$$
\frac{1}{p_{(i+1)}} \leq \frac{1}{5\left(p_{(i+1)}\right)}+\frac{\log p_{(i+1)}}{\log \left(\log p_{(i+1)} \sharp\right)}-1
$$

Consequently this implies that Theorem 5.11 is satisfied for all $p_{i} \in \mathbb{N} \mid p_{i} \geq 37$, a simple computer calculation verifies that this inequality also holds within the interval $13 \leq p_{i} \leq 37$. This necessarily means that Theorem 5.11 is satisfied for all $p_{i} \in \mathbb{N} \mid p_{i} \geq 13$, thus completing the proof.

Theorem 5.12 (The Step Sequence Estimation Error Upper Bound).

The inverse of a root of the pertinent prime number at every step exceeds the value of the estimation error of the tailored logarithmic integral TL $i_{(n)}$ step sequence $\forall p_{i} \in \mathbb{N} \mid p_{i} \geq 13$ :

$$
\operatorname{SER}_{\left(p_{(i+1)}\right)}=\left(\int_{\theta_{1}}^{\theta_{2}} \frac{d t}{\log t}\right)-1<\frac{1}{\sqrt[a]{p_{(i+1)}}} \quad \text { where } a=\frac{\pi}{2}
$$


where $p_{(i)}$ and $p_{(i+1)}$ are associated with the lower/upper limit of integration $\theta_{1}$ and $\theta_{2}$ respectively.

Proof.

By Lemma 5.9 the sequence $\mathcal{S E \mathcal { R }}\left(p_{(i+1)}\right)$ is Cauchy and it converges from above to the limit $\mathcal{L}=0$. The sequence of the reciprocals of the root of prime numbers is clearly Cauchy and converges to the limit $\mathcal{L}=0$. By Lemma 5.9 we have that:

$$
\int_{\theta_{1}}^{\theta_{2}} \frac{d t}{\log t}-1 \leq \frac{1}{p_{(i+1)}}+\frac{\log p_{(i+1)}}{\log \left(\log p_{(i+1)} \sharp\right)}-1
$$$$
\text { for all } p \in \mathbb{N} \mid p \geq 13
$$

Consequently Theorem 5.12 is valid if and only if:

$$
\frac{1}{p_{(i+1)}}+\frac{\log p_{(i+1)}}{\log \left(\log p_{(i+1)} \sharp\right)}-1 \leq \frac{1}{\sqrt[a]{p_{(i+1)}}} \quad \text { where } a=\frac{\pi}{2}
$$

Now,

$$
\begin{aligned}
& (5.32) \frac{1}{\left(p_{(i+1)}\right)}+\frac{\log p_{(i+1)}}{\log \left(\log p_{(i+1)} \sharp\right)}-1-\frac{1}{\sqrt[a]{p_{(i+1)}}}= \\
& \frac{\left(p_{(i+1)} \sqrt[a]{p_{(i+1)}}\left(\log \left(p_{(i+1)}\right)\right)\right)-\left(p_{(i+1)}-\sqrt[a]{p_{(i+1)}}+p_{(i+1)} \sqrt[a]{p_{(i+1)}}\right)\left(\log \left(\log \left(p_{(i+1)} \sharp\right)\right)\right)}{\left(p_{(i+1)} \sqrt[a]{p_{(i+1)}}\right)\left(\log \left(\log \left(p_{(i+1)} \sharp\right)\right)\right)}
\end{aligned}
$$

From Lemma 4.3 we have that $\log p_{(i+1)} \sharp$ is asymptotic (from below):

$$
\log p_{(i+1)} \sharp \sim p_{(i+1)}
$$

as well as:

$$
\log p_{(i+1)} \sharp<p_{(i+1)} \leq n \quad \forall n \in \mathbb{N} \mid n \geq 2
$$

Hence,

$$
\log \left(\log p_{(i+1)} \sharp\right) \leq \log p_{(i+1)}
$$

From Lemma 4.5 we have for all $p_{(i+1)} \in \mathbb{N} \mid p_{(i+1)} \geq 2$ :

$$
\mathcal{U} \mathcal{B}_{p_{(i+1)}}=2 \sqrt{p_{(i+1)}}>\left(p_{(i+1)}-\log p_{(i+1)} \sharp\right)
$$

Consequently, from the above we obtain:

$$
\left(p_{(i+1)}-\mathcal{U} \mathcal{B}_{p_{(i+1)}}\right)<\log p_{(i+1)} \sharp
$$

Bearing in mind that for all positive $a, b \in \mathbb{R} \mid a>b$ :

$$
\log (a+b)=\log (a(1+b / a))=\log (a)+\log \left(1+\frac{b}{a}\right)
$$

Thus, by Lemma 4.3 we have:

$$
p_{(i+1)} \sqrt[a]{p_{(i+1)}}\left(\log \left(p_{(i+1)}-\mathcal{U} \mathcal{B}_{p_{(i+1)}}\right)\right) \leq p_{(i+1)} \sqrt[a]{p_{(i+1)}} \log \left(\log \left(p_{(i+1)} \sharp\right)\right)
$$


Hence,

$$
\begin{array}{r}
p_{(i+1)} \sqrt[a]{p_{(i+1)}}\left(\log \left(p_{(i+1)}\right)\right)=p_{(i+1)} \sqrt[a]{p_{(i+1)}}\left(\log \left(p_{(i+1)}-\mathcal{U B}_{p_{(i+1)}}+\mathcal{U} \mathcal{B}_{p_{(i+1)}}\right)\right) \\
\left.=p_{(i+1) \sqrt[a]{p_{(i+1)}}\left[\log \left(p_{(i+1)}-\mathcal{U} \mathcal{B}_{p_{(i+1)}}\right)\right.}+\log \left(1+\frac{\mathcal{U} \mathcal{B}_{p_{(i+1)}}}{\left(p_{(i+1)}-\mathcal{U} \mathcal{B}_{p_{(i+1)}}\right)}\right)\right] \\
\leq p_{(i+1)} \sqrt[a]{p_{(i+1)}}\left(\log \left(\log \left(p_{(i+1)} \sharp\right)\right)\right)
\end{array}
$$

Suppose that the Theorem 5.12 is false. Then it must be true that the numerator of equation 5.32 is greater than zero. From inequality 5.37 we see that without loss of generality, upon substitution into the numerator of the inequality 5.32 , we can drop the common terms obtaining:

$$
\begin{aligned}
& \left(p_{(i+1)} \sqrt[a]{p_{(i+1)}}\left(\log \left(p_{(i+1)}\right)\right)\right)-\left(p_{(i+1)}-\sqrt[a]{p_{(i+1)}}+p_{(i+1)} \sqrt[a]{p_{(i+1)}}\right)\left(\log \left(\log \left(p_{(i+1)} \sharp\right)\right)\right) \\
& \leq p_{(i+1)} \sqrt[a]{p_{(i+1)}} \log \left(1+\frac{\mathcal{U} \mathcal{B}_{p_{(i+1)}}}{\left(p_{(i+1)}-\mathcal{U} \mathcal{B}_{p_{(i+1)}}\right)}\right)-\left(p_{(i+1)}-\sqrt[a]{p_{(i+1)}}\right)\left(\log \left(\log \left(p_{(i+1)} \sharp\right)\right)\right)
\end{aligned}
$$

However at $p_{(i+1)}=197$ the difference 5.38 attains $\sim-1.20860443$ and diverges. Hence we have a contradiction to our hypothesis which implies that the inequality is true:

$$
\frac{1}{p_{(i+1)}}+\frac{\log p_{(i+1)}}{\log \left(\log p_{(i+1)} \sharp\right)}-1 \leq \frac{1}{\sqrt[a]{p_{(i+1)}}} \quad \text { where } a=\frac{\pi}{2}
$$

Consequently this implies that Theorem 5.12 is satisfied for all $p_{i} \in \mathbb{N} \mid p_{i} \geq 197$, a simple computer calculation verifies that this inequality also holds within the interval $13 \leq p_{i} \leq 197$. This necessarily means that Theorem 5.12 is satisfied for all $p_{i} \in \mathbb{N} \mid p_{i} \geq 13$, thus completing the proof.

Hence, by Theorems 5.11 and 5.12 , for the largest prime number $p_{(i+1)}$ that satisfies the condition $p_{(i+1)} \leq n \in \mathbb{N}$, we have:

$$
\frac{1}{p_{(i+1)}}<\left(\int_{\theta_{1}}^{\theta_{2}} \frac{d t}{\log t}\right)-1<\frac{1}{\sqrt[a]{p_{(i+1)}}} \quad \forall p_{(i)} \geq 13 \quad \text { where } a=\frac{\pi}{2}
$$

\section{Remark 5.5.}

We need to re-define the lower/upper limits of integration to conform with the summation limits. The computation of the sum of step errors of the integral $T L i_{n}$ begins at $p_{2}=3$, irrespective of the fact that the computation of the sums pertinent to the bounds (Infimum, Supremum, Lower and Upper) begins first at $p_{15}=47$.

Definition 5.13 (Theta applicable for summation). $\theta_{1}=\log \left(p_{(2+(k-1))} \sharp\right)$

Definition 5.14 (Theta applicable for summation). $\theta_{2}=\log \left(p_{(2+k) \sharp)}\right)$ 
Theorem 5.15 $\left(T L i_{(n)}\right.$ Estimation Error Divergence).

The error arising in the estimation of the prime counting function $\pi_{(n)}$ by the application of the tailored logarithmic integral $T L i_{(n)}$, diverges to infinity:

$\lim _{n \rightarrow \infty}\left(T L i_{(n)}-\pi_{(n)}\right)=\lim _{p_{(n)} \rightarrow \infty}\left(\sum_{(k=1)}^{\left(\pi_{(n)}-2\right)}\left\{\left(\int_{\theta_{1}}^{\theta_{2}} \frac{d t}{\log t}\right)-1\right\}+\left(\int_{2}^{\log 6} \frac{d t}{\log t}-\pi_{3}\right)\right) \rightarrow \infty$

Where the limits of integration $\theta_{1}$ and $\theta_{2}$ are given by the Definitions 5.13 and 5.14 respectively. Besides, the prime number $p_{(n)}$ is defined as being the biggest prime $p \leq n$. Furthermore,

$$
\begin{gathered}
\left(T L i_{(n)}-\pi_{(n)}\right)>\sum_{p \leq n} \frac{1}{p} \forall n \in \mathbb{N} \mid n \geq 983 \\
T L i_{(n)}=\int_{2}^{\theta_{(n)}} \frac{d t}{\log t}>\pi_{(n)} \quad \forall n \in \mathbb{N} \mid n \geq 43
\end{gathered}
$$

Proof.

By Lemma 5.9 for all $p_{i} \in \mathbb{N} \mid p_{i} \geq 13$ the relation holds at every step:

$$
\left\{\left(\int_{\theta_{1}}^{\theta_{2}} \frac{d t}{\log t}\right)-1\right\}>\frac{1}{p_{(i+1)}}
$$

Because the sum of reciprocals of successive prime numbers diverges, consequently, the sum:

$$
\lim _{p_{(n)} \rightarrow \infty}\left(\sum_{(k=1)}^{\left(\pi_{(n)}-2\right)}\left\{\left(\int_{\theta_{1}}^{\theta_{2}} \frac{d t}{\log t}\right)-1\right\}\right) \rightarrow \infty
$$

must necessarily diverge, by comparison with the divergent sum of reciprocals of successive prime numbers. The complete estimation error of the tailored integral is given by:

$$
\left(T L i_{(n)}-\pi_{(n)}\right)=\left(\sum_{(k=1)}^{\left(\pi_{(n)}-2\right)}\left\{\left(\int_{\theta_{1}}^{\theta_{2}} \frac{d t}{\log t}\right)-1\right\}+\left(\int_{2}^{\log 6} \frac{d t}{\log t}-\pi_{3}\right)\right)
$$

Consequently therefore, by the divergence of the sum 5.44, the estimation error of the tailored integral 5.45 must necessarily diverge:

$$
\lim _{n \rightarrow \infty}\left(T L i_{(n)}-\pi_{(n)}\right)=\lim _{p_{(n)} \rightarrow \infty}\left(\sum_{(k=1)}^{\left(\pi_{(n)}-2\right)}\left\{\left(\int_{\theta_{1}}^{\theta_{2}} \frac{d t}{\log t}\right)-1\right\}+\left(\int_{2}^{\theta_{3}} \frac{d t}{\log t}-\pi_{3}\right)\right) \rightarrow \infty
$$

In fact the sum of reciprocals of successive prime numbers and 5.45 intersect. Direct calculation at $n=983$ shows that the difference:

$$
\left(T L i_{(n)}-\pi_{(n)}\right)-\sum_{p \leq n} \frac{1}{p} \approx 0.0004199365947656908
$$


Because by Lemma 5.9 the inequality 5.43 holds for all $p_{i} \in \mathbb{N} \mid p_{i} \geq 13$, this implies that:

$$
\left(T L i_{(n)}-\pi_{(n)}\right)>\sum_{p \leq n} \frac{1}{p} \forall n \in \mathbb{N} \mid n \geq 983
$$

Direct computation verifies that the estimation error of $T L i_{(n)}$ :

$$
\left(T L i_{(n)}-\pi_{(n)}\right)>0 \quad \text { for } n \in \mathbb{N} \mid 43<n \geq 983
$$

At $n=43$ the estimation error of $T L i_{(n)}$ attains the value of 0.002993180461560385 . Therefore, by the divergence of the tailored integral estimation error, it must be true that:

$$
T L i_{(n)}=\int_{2}^{\theta_{(n)}} \frac{d t}{\log t}>\pi_{(n)} \quad \forall n \in \mathbb{N} \mid n \geq 43
$$

This concludes the proof of Theorem 5.15.

\subsection{Supremum and Infimum estimation error bounds on $T L i_{(n)}-\pi_{(n)}$.}

\section{Remark 5.6.}

The estimation error of the $T L i_{(n)}$ increases stepwise at the primes. Since however the magnitude of the increase is small, as a result the curve presents itself as a rising virtually smooth slope. It absolutely lacks the large amplitude variation, which is the intrinsic characteristic of the Gauss' offset $L i_{(n)}$. Please refer to Fig. 4. The Infimum and Supremum estimation error bounds, in conjunction with the tailored logarithmic integral, give us both the most accurate estimate of $\pi_{(n)}$ and the best estimation error bounds. The drawback is, that the formulae are computationally quite demanding.

Theorem 5.16 (Infimum Estimation Error Bound).

The error of estimation of the prime counting function $\pi_{(n)}$ by the application of the tailored logarithmic integral $T L i_{(n)}$, for all $n \in \mathbb{N} \mid n \geq 47$ is bounded below by a divergent sum:

$$
\begin{aligned}
\mathcal{L B}_{(n)}= & \sum_{k=1}^{\left(\pi_{(n)}-14\right)} \frac{1}{p_{(14+k)}}<\mathcal{I} \mathcal{N} \mathcal{F}_{(n)}=\sum_{k=1}^{\left(\pi_{(n)}-14\right)}\left\{\frac{\log p_{(14+k)}}{\log \left(\log p_{(14+k)} \sharp\right)}+\frac{1}{5\left(p_{(14+k)}\right)}-1\right\} \\
& <\left(\sum_{(k=1)}^{\left(\pi_{(n)}-2\right)}\left\{\int_{\theta_{1}}^{\theta_{2}} \frac{d t}{\log t}-1\right\}+\left(\int_{2}^{\log 6} \frac{d t}{\log t}-\pi_{3}\right)\right)=\left(T L i_{(n)}-\pi_{(n)}\right)
\end{aligned}
$$

Further, the limits of integration $\theta_{1}, \theta_{2}$ implement Definition 5.13 and 5.14 respectively, while $p_{(n)}$ is the greatest prime $p \in \mathbb{N} \mid p \leq n$.

Proof.

Let's consider both $\mathcal{L B}_{(n)}$ and $\mathcal{I N F}_{(n)}$ stepwise first. Evidently, both of them are Cauchy, convergent to zero sequences, while the lower bound function $\mathcal{L B}_{(n)}$ is strictly monotonic. Further, by Theorem 5.11 we have that:

$$
\frac{1}{p_{(i+1)}} \leq \frac{1}{5\left(p_{(i+1)}\right)}+\frac{\log p_{(i+1)}}{\log \left(\log p_{(i+1)} \sharp\right)}-1
$$


The relation holds stepwise at every step for all $p_{(n)} \in \mathbb{N} \mid p_{(n)} \geq 13$. Adding simultaneously the terms of both sequences and forming two respective sums, does not invalidate the relation. Considering in turn stepwise the functions $\mathcal{I} \mathcal{N} \mathcal{F}_{(n)}$ and the step sequence $T L i_{(n)}$, evidently both are Cauchy, convergent to zero sequences. Further by Lemma 5.9 and Corollary 5.10 we have that at every step:

$$
\frac{\log p_{(i+1)}}{\log \left(\log p_{(i+1)} \sharp\right)}+\frac{1}{5\left(p_{(i+1)}\right)}-1 \leq\left[\int_{\theta_{1}}^{\theta_{2}} \frac{d t}{\log t}-1\right] \quad \text { for all } p \in \mathbb{N} \mid p \geq 13
$$

The inclusion of the additional, first step term:

$$
\int_{2}^{\log 6} \frac{d t}{\log t}-\pi_{3}=\int_{2}^{\log 6} \frac{d t}{\log t}-2 \approx-2.3266013098834977
$$

has clearly the effect of shifting the curve of the $\left(T L i_{(n)}-\pi_{n}\right)$ significantly down, thereby upsetting the stepwise inter-relationships. However, by Theorem 5.15 we have that:

$$
\begin{aligned}
& \left(T L i_{(n)}-\pi_{(n)}\right)= \\
& \left(\sum_{(k=1)}^{\left(\pi_{(n)}-2\right)}\left\{\int_{\theta_{1}}^{\theta_{2}} \frac{d t}{\log t}-1\right\}+\left(\int_{2}^{\log 6} \frac{d t}{\log t}-\pi_{3}\right)\right)=\left(\int_{2}^{\theta_{(n)}} \frac{d t}{\log t}\right)-\pi_{(n)}>0 \\
& \forall n \in \mathbb{N} \mid n \geq 43
\end{aligned}
$$

with $\theta_{(n)}$ given by the Definition 5.1. Thus, at $p_{n}=43$ the tailored integral concludes the stage of recovery instigated by the addition of the initial term, vide equation 5.48 above. The difference:

$$
\left(\sum_{k=1}^{\left(\pi_{(n)}-14\right)} \frac{1}{p_{(14+k)}}\right)-\left(\sum_{k=1}^{\left(\pi_{(n)}-14\right)}\left\{\frac{\log p_{(14+k)}}{\log \left(\log p_{(14+k) \sharp)}\right.}+\frac{1}{5\left(p_{(14+k)}\right)}-1\right\}\right)
$$

at $p_{15}=47$ attains $\sim-0.0200272$ and diverges decisively. Consequently, by Theorem 5.11 it must be true that the relation 5.50 holds for all $p_{(n)} \in \mathbb{N} \mid p_{(n)} \geq 47$. The difference:

$$
\left(\sum_{k=1}^{\left(\pi_{(n)}^{-14)}\right.}\left\{\frac{\log p_{(14+k)}}{\log \left(\log p_{(14+k)} \sharp\right)}+\frac{1}{5\left(p_{(14+k)}\right)}-1\right\}\right)-\left(T L i_{(n)}-\pi_{(n)}\right)
$$

at $p_{15}=47$ the difference attains $\sim-0.0125411$ and further diverges. Since the relation 5.47 holds stepwise for all $p_{(n)} \in \mathbb{N} \mid p_{(n)} \geq 13$, therefore, necessarily it must be true that the relation 5.51 holds for all $p_{(n)} \in \mathbb{N} \mid p_{(n)} \geq 47$. Consequently, for all $p_{(n)} \in \mathbb{N} \mid p_{(n)} \geq 47$ we obtain:

$$
\begin{aligned}
& \sum_{k=1}^{\left(\pi_{(n)}-14\right)} \frac{1}{p_{(14+k)}}<\sum_{k=1}^{\left(\pi_{(n)}-14\right)}\left\{\frac{\log p_{(14+k)}}{\log \left(\log p_{(14+k)} \sharp\right)}+\frac{1}{5\left(p_{(14+k)}\right)}-1\right\} \\
& \quad<\left(\sum_{(k=1)}^{\left(\pi_{(n)}-2\right)}\left\{\int_{\theta_{1}}^{\theta_{2}} \frac{d t}{\log t}-1\right\}+\left(\int_{2}^{\log 6} \frac{d t}{\log t}-\pi_{3}\right)\right)=\left(\int_{2}^{\theta_{(n)}} \frac{d t}{\log t}\right)-\pi_{(n)}
\end{aligned}
$$

This concludes the proof of Theorem 5.16. 


\section{Remark 5.7.}

Inclusion of the additional, initial term:

$$
\int_{2}^{\log 6} \frac{d t}{\log t}-\pi_{3}=\int_{2}^{\log 6} \frac{d t}{\log t}-2 \approx-2.3266013098834977
$$

to complete the domain of integration, when summing the step terms of the $T L i_{n}$ up to some predetermined $n \in \mathbb{N}$, has a drawback. It upsets the established stepwise balance with all its bounds. To resolve the issue decisively with the bounds, we have to drop 14 of the initial terms of the sum, for each bound. This way, the balance in their inter-relationships is restored, as $\left(T L i_{(n)}-\pi_{(n)}\right)>0 \quad \forall n \in \mathbb{N} \mid n \geq 43$.

Theorem 5.17 (Supremum Estimation Error Bound).

The error of estimation of the prime counting function $\pi_{(n)}$ by the application of the tailored logarithmic integral $T L i_{(n)}$, for all $n \in \mathbb{N} \mid n \geq 47$ is bounded above by a divergent sum:

$$
\begin{aligned}
& T L i_{(n)}-\pi_{(n)}<\mathcal{S U P R}_{(n)} \\
& =\sum_{(k=1)}^{\left(\pi_{(n)}-14\right)}\left\{\frac{\log p_{(14+k)}}{\log \left(\log p_{(14+k)} \sharp\right)}+\frac{1}{p_{(14+k)}}-1\right\}<\sum_{(k=1)}^{\left(\pi_{(n)}-14\right)} \frac{1}{\sqrt[a]{p_{(14+k)}}}=\mathcal{U} \mathcal{B}_{(n)}
\end{aligned}
$$

where $a=\frac{\pi}{2}$, while $p_{(n)}$ is the biggest prime number $p \leq n$.

Proof.

Let's consider both $\mathcal{U B}_{(n)}$ and $\mathcal{S U P \mathcal { R }}(n)$ stepwise first. Evidently, both of them are Cauchy, convergent to zero sequences, while the upper bound function $\mathcal{U} \mathcal{B}_{(n)}$ is strictly monotonic. Further, by Theorem 5.12 we have that:

$$
\frac{1}{\sqrt[a]{p_{(i+1)}}} \geq \frac{1}{\left(p_{(i+1)}\right)}+\frac{\log p_{(i+1)}}{\log \left(\log p_{(i+1)} \sharp\right)}-1 \quad \text { where } a=\frac{\pi}{2}
$$

the relation holds stepwise at every step for all $p_{(n)} \in \mathbb{N} \mid p_{(n)} \geq 13$. Adding simultaneously the terms of both sequences and forming two respective sums, does not invalidate the relation. Considering in turn stepwise the functions $\mathcal{I} \mathcal{N} \mathcal{F}_{(n)}$ and the step sequence $T L i_{(n)}$, evidently both are Cauchy, convergent to zero sequences. Further by Lemma 5.9 and Corollary 5.10 we have that at every step:

$$
\frac{\log p_{(i+1)}}{\log \left(\log p_{(i+1)} \sharp\right)}+\frac{1}{\left(p_{(i+1)}\right)}-1 \geq\left[\int_{\theta_{1}}^{\theta_{2}} \frac{d t}{\log t}-1\right] \quad \text { for all } p \in \mathbb{N} \mid p \geq 13
$$

The inclusion of the additional, first step term:

$$
\int_{2}^{\log 6} \frac{d t}{\log t}-\pi_{3}=\int_{2}^{\log 6} \frac{d t}{\log t}-2 \approx-2.3266013098834977
$$

has clearly the effect of shifting the curve of the $\left(T L i_{(n)}-\pi_{n}\right)$ significantly down, thereby upsetting the stepwise inter-relationships. However, by Theorem 5.15 we 
have that:

$$
\begin{aligned}
& \left(T L i_{(n)}-\pi_{(n)}\right)= \\
& \left(\sum_{(k=1)}^{\left(\pi_{(n)}-2\right)}\left\{\int_{\theta_{1}}^{\theta_{2}} \frac{d t}{\log t}-1\right\}+\left(\int_{2}^{\log 6} \frac{d t}{\log t}-\pi_{3}\right)\right)=\left(\int_{2}^{\theta_{(n)}} \frac{d t}{\log t}\right)-\pi_{(n)}>0 \\
& \forall n \in \mathbb{N} \mid n \geq 43
\end{aligned}
$$

with $\theta_{(n)}$ given by the Definition 5.1. Thus, at $p_{n}=43$ the tailored integral concludes the stage of recovery instigated by the addition of the initial term, vide equation 5.55 above. The difference:

$$
\left(\sum_{k=1}^{\left(\pi_{(n)}-14\right)} \frac{1}{\sqrt[a]{p_{(14+k)}}}\right)-\left(\sum_{k=1}^{\left(\pi_{(n)}-14\right)}\left\{\frac{\log p_{(14+k)}}{\log \left(\log p_{(14+k) \sharp)}\right.}+\frac{1}{\left(p_{(14+k)}\right)}-1\right\}\right)
$$

at $p_{15}=47$ attains $\sim 0.0278753$ and diverges decisively. Consequently, by Theorem 5.12 it must be true that the relation 5.57 holds for all $p_{(n)} \in \mathbb{N} \mid p_{(n)} \geq 47$. The difference:

$$
\left(\sum_{k=1}^{\left(\pi_{(n)}-14\right)}\left\{\frac{\log p_{(14+k)}}{\log \left(\log p_{(14+k)} \sharp\right)}+\frac{1}{\left(p_{(14+k)}\right)}-1\right\}\right)-\left(T L i_{(n)}-\pi_{(n)}\right)
$$

at $p_{15}=47$ the difference attains $\sim 0.00448015$ and further diverges. Since the relation 5.54 holds stepwise for all $p_{(n)} \in \mathbb{N} \mid p_{(n)} \geq 13$, therefore, necessarily it must be true that the relation 5.58 holds for all $p_{(n)} \in \mathbb{N} \mid p_{(n)} \geq 47$. Consequently, for all $p_{(n)} \in \mathbb{N} \mid p_{(n)} \geq 47$ we obtain:

$$
\begin{aligned}
& \sum_{k=1}^{\left(\pi_{(n)}-14\right)} \frac{1}{\sqrt[a]{p_{(14+k)}}}>\sum_{k=1}^{\left(\pi_{(n)}-14\right)}\left\{\frac{\log p_{(14+k)}}{\log \left(\log p_{(14+k)} \sharp\right)}+\frac{1}{\left(p_{(14+k)}\right)}-1\right\} \\
& >\left(\sum_{(k=1)}^{\left(\pi_{(n)}-2\right)}\left\{\int_{\theta_{1}}^{\theta_{2}} \frac{d t}{\log t}-1\right\}+\left(\int_{2}^{\log 6} \frac{d t}{\log t}-\pi_{3}\right)\right)=\left(\int_{2}^{\theta_{(n)}} \frac{d t}{\log t}\right)-\pi_{(n)}
\end{aligned}
$$

Thus, concluding the proof.

Therefore on the basis of Theorems 5.16 and 5.17, the relation holds for all prime numbers $p \in \mathbb{N} \mid p \geq 47$ :

$$
\begin{aligned}
& \sum_{k=1}^{\left(\pi_{(n)}-14\right)} \frac{1}{p_{(14+k)}}<\sum_{k=1}^{\left(\pi_{(n)}-14\right)}\left\{\frac{\log p_{(14+k)}}{\log \left(\log p_{(14+k)} \sharp\right)}+\frac{1}{5\left(p_{(14+k)}\right)}-1\right\} \\
& \left.<\sum_{(k=1)}^{\left(\pi_{(n)}-2\right)}\left\{\int_{\theta_{1}}^{\theta_{2}} \frac{d t}{\log t}-1\right\}+\left(\int_{2}^{\log 6} \frac{d t}{\log t}-\pi_{3}\right)\right)=\left(\int_{2}^{\theta_{(n)}} \frac{d t}{\log t}\right)-\pi_{(n)} \\
& <\sum_{k=1}^{\left(\pi_{(n)}-14\right)}\left\{\frac{\log p_{(14+k)}}{\log \left(\log p_{(14+k)} \sharp\right)}+\frac{1}{\left(p_{(14+k)}\right)}-1\right\}<\sum_{k=1}^{\left(\pi_{(n)}-14\right)} \frac{1}{\sqrt[a]{p_{(14+k)}}} \text { where } a=\frac{\pi}{2}
\end{aligned}
$$


5.6. The elementary bounds on the prime counting function $\pi_{n}$.

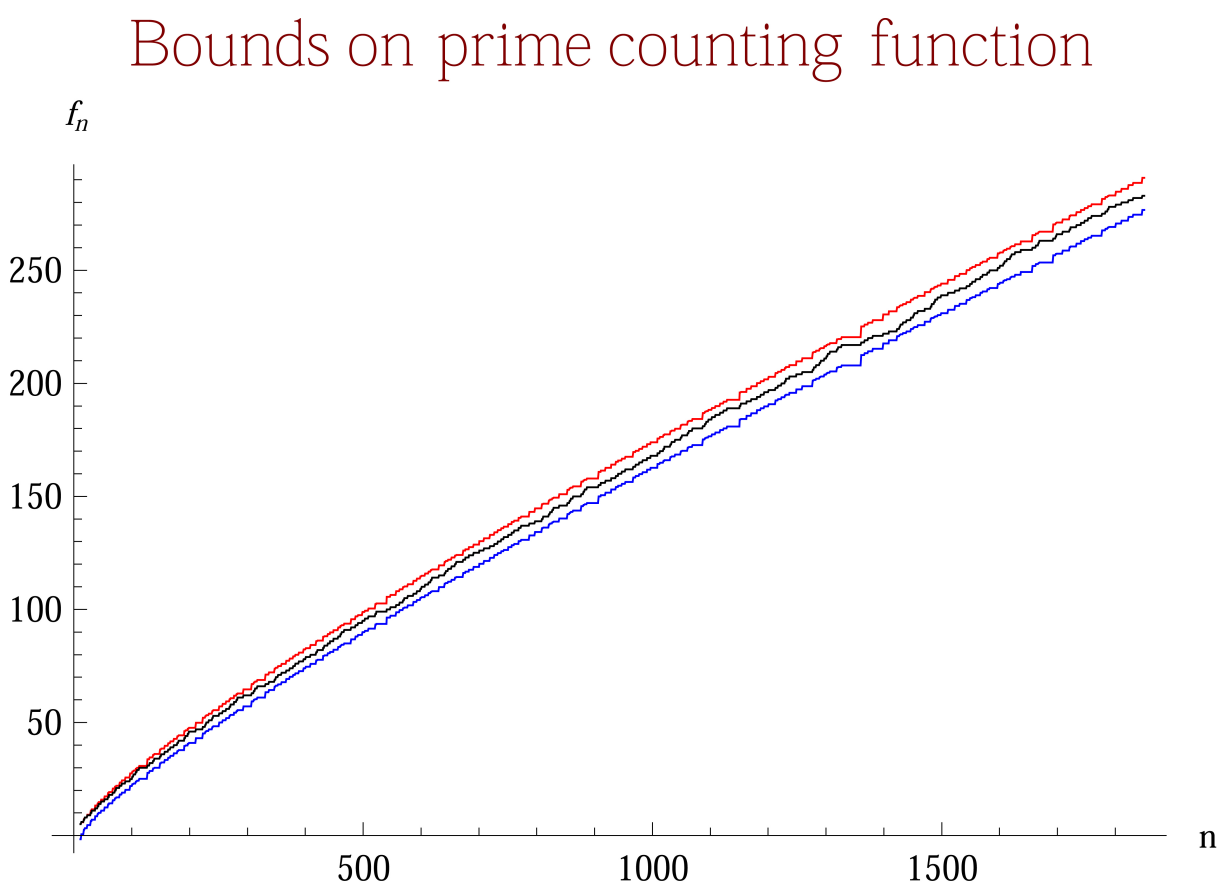

Figure 7. The drawing shows the Upper (Red) and Lower (Blue) bounds on $\pi_{n}$ (Black). The figure is drawn at every $n \in \mathbb{N}$ in the range $11 \leq 1850$.
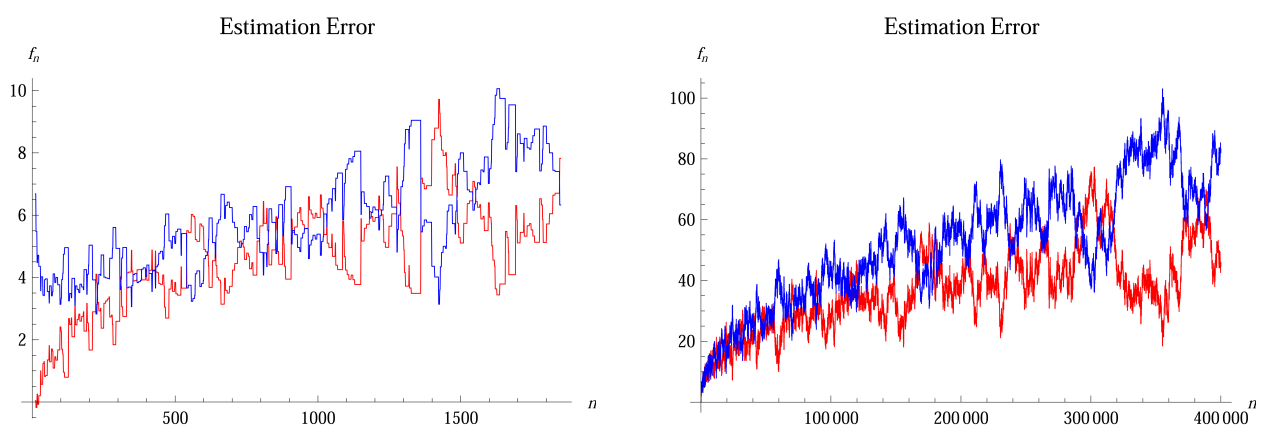

Figure 8. The drawing shows the estimation error made by the Upper (Red) and Lower (Blue) bounds of the prime counting function $\pi_{n}$. The L.H. figure is drawn at every $n \in \mathbb{N}$ in the range $11 \leq 1850$, while the R.H. figure is drawn at every $n \in \mathbb{N}$ in the range $11 \leq 400009$.

The calculation of the integral $T L i_{(n)}$ may become computationally quite demanding for larger values of $n \in \mathbb{N}$. This attribute of computation of the tailored 
integral $T L i_{(n)}$ pertains to the sequential, exhaustive process of calculation of the value of $\log p_{(n)} \sharp$, which has exponential time complexity. Therefore, at the cost of an increased estimation error, this section presents a method to obtain the approximate value of the Supremum/Infimum in an efficient manner.

Definition 5.18 (Upper Bound integration limit).

$$
\begin{aligned}
\theta_{\mathcal{U}}=\left(p_{(n)}-(\sqrt{5}-1)\left(4 \gamma^{2}-2 \gamma\right)\left(\log p_{(n)}\right) \sqrt[3]{p_{(n)}}\right) & \\
& \text { where the prime } p_{n} \in \mathbb{N} \mid p_{n} \geq 11
\end{aligned}
$$

Definition 5.19 (Lower Bound integration limit).

$$
\theta_{\mathcal{L}}=p_{(n)}-\left(\exp \left(\gamma+\frac{1}{2}\right)\right) \sqrt{p_{n}} \quad \text { where the prime } p_{n} \in \mathbb{N} \mid p_{n} \geq 11
$$

Theorem 5.20 (Upper and Lower Bounds on $\pi_{n}$ ).

Let $\pi_{n}$ be the prime counting function. Then, the upper and lower bounds on $\pi_{n}$ are given by:

$$
\begin{gathered}
L B_{n}=\int_{2}^{\theta_{\mathcal{L}}} \frac{d t}{\log t}<\pi_{n} \quad \forall n \in \mathbb{N} \mid n \geq 11 \\
\pi_{n}<\int_{2}^{\theta_{\mathcal{U}}} \frac{d t}{\log t}+C_{1}=U B_{n} \quad \forall n \in \mathbb{N} \mid n \geq 23
\end{gathered}
$$

With the constant $C_{1}=0$. Setting $C_{1}=0.2$ will include all $n \in \mathbb{N} \mid 11 \leq n \leq 23$, it will however interfere with subsequent results.

\section{Remark 5.8.}

The proof is a multistep process and will be carried over several of the following theorems/lemmas. The proof pertains to the Upper Bound only, an analogous process needs to be carried out in the case of the Lower Bound, which is left at the discretion of the reader.

By Lemma 4.5 we have:

$$
\begin{aligned}
\log p_{(n)} \sharp<\left(p_{(n)}-\right. & \left.(\sqrt{5}-1)\left(4 \gamma^{2}-2 \gamma\right)\left(\log p_{(n)}\right) \sqrt[3]{p_{(n)}}\right) \\
& \text { where } p_{n} \text { is the greatest prime number } p \in \mathbb{N} \mid p \leq n
\end{aligned}
$$

At the cost of an increased estimation error we may estimate the true value of the tailored integral quite easily.

Theorem 5.21 (Divergence Of The Difference $\left.L i_{(n)}-U B_{(n)}\right)$.

The difference in values taken by the logarithmic integral $L i_{(n)}$ and the estimate of the tailored logarithmic integral $U B_{(n)}$ given by Theorem 5.20 , for all $n \in \mathbb{N} \mid n \geq$ 11, increases without a bound as $n$ tends to infinity:

$$
\lim _{n \rightarrow \infty}\left(L i_{(n)}-U B_{(n)}\right)=\lim _{n \rightarrow \infty}\left(\int_{\theta_{\mathcal{U}}}^{n} \frac{d t}{\log t}\right) \rightarrow \infty
$$


Consequently, the value of the $U B_{(n)}$ will always remain less than the value obtained by the Gauss' $L i_{(n)}$ :

$$
L i_{(n)}=\int_{2}^{n} \frac{d t}{\log t}>U B_{(n)}=\int_{2}^{\theta_{\mathcal{U}}} \frac{d t}{\log t} \quad \forall n \in \mathbb{N} \mid n \geq 11
$$

Proof.

Clearly,

$$
\left(p_{(n)}-(\sqrt{5}-1)\left(4 \gamma^{2}-2 \gamma\right)\left(\log p_{(n)}\right) \sqrt[3]{p_{(n)}}\right)<p_{(n)} \leq n \quad \forall n \in \mathbb{N} \mid n \geq 3
$$

where $p_{(n)}$ is the largest prime $p \leq n$. Since the limit diverges:

$$
\lim _{n \rightarrow \infty}\left((\sqrt{5}-1)\left(4 \gamma^{2}-2 \gamma\right)\left(\log p_{(n)}\right) \sqrt[3]{p_{(n)}}\right) \rightarrow \infty
$$

the difference between the pertinent intervals of computation of $L i_{(n)}$ and $U B_{(n)}$ increases unboundedly. Due to the fact that,

$$
(\sqrt{5}-1)\left(4 \gamma^{2}-2 \gamma\right)(\log n)>1 \quad \forall n \in \mathbb{N} \mid n \geq 94
$$

the difference between the intervals of computation increases at a rate proportional to $k\left(\log p_{(n)}\right) \sqrt[3]{p_{(n)}}$ where $k \approx 0.220367$ for all $n \in \mathbb{N} \mid n \geq 94$. The exact difference between the intervals of computation of $L i_{(n)}$ and $U B_{(n)}$ is given by:

$$
\left(n-\theta_{\mathcal{U}}\right)=(\sqrt{5}-1)\left(4 \gamma^{2}-2 \gamma\right)\left(\log p_{(n)}\right) \sqrt[3]{p_{(n)}}+\left(n-p_{(n)}\right)
$$

However, because the difference $\left(n-p_{(n)}\right)$ is bounded above by the maximal prime gaps Supremum given by Theorem 3.6, its contribution for large $n$ is negligible. Therefore, we may drop the difference $\left(n-p_{(n)}\right)$ and by an application of the PNT we obtain an estimate of the true value of the minimum difference between the two integrals:

$$
\int_{\theta_{\mathcal{U}}}^{n} \frac{d t}{\log t} \gtrsim \frac{(\sqrt{5}-1)\left(4 \gamma^{2}-2 \gamma\right)\left(\log p_{(n)}\right) \sqrt[3]{p_{(n)}}}{\log p_{(n)}}=(\sqrt{5}-1)\left(4 \gamma^{2}-2 \gamma\right) \sqrt[3]{p_{(n)}}
$$

The estimate 5.69 clearly increases monotonically without a bound. Consequently,

$$
\lim _{n \rightarrow \infty}\left(\int_{\theta_{\mathcal{U}}}^{n} \frac{d t}{\log t}\right)=\lim _{n \rightarrow \infty}\left(\frac{\left(n-\theta_{\mathcal{U}}\right)}{\log n}\right) \rightarrow \infty
$$

At $n=11$ the difference:

$$
\left(L i_{(n)}-U B_{(n)}\right)=\int_{\theta_{\mathcal{U}}}^{n} \frac{d t}{\log t} \approx 0.501785
$$

and increases as $\mathrm{n}$ tends to infinity. This implies,

$$
L i_{(n)}=\int_{2}^{n} \frac{d t}{\log t}>U B_{(n)}=\int_{2}^{\theta \mathcal{U}} \frac{d t}{\log t} \quad \forall n \in \mathbb{N} \mid n \geq 11
$$

Thus completing the proof. 

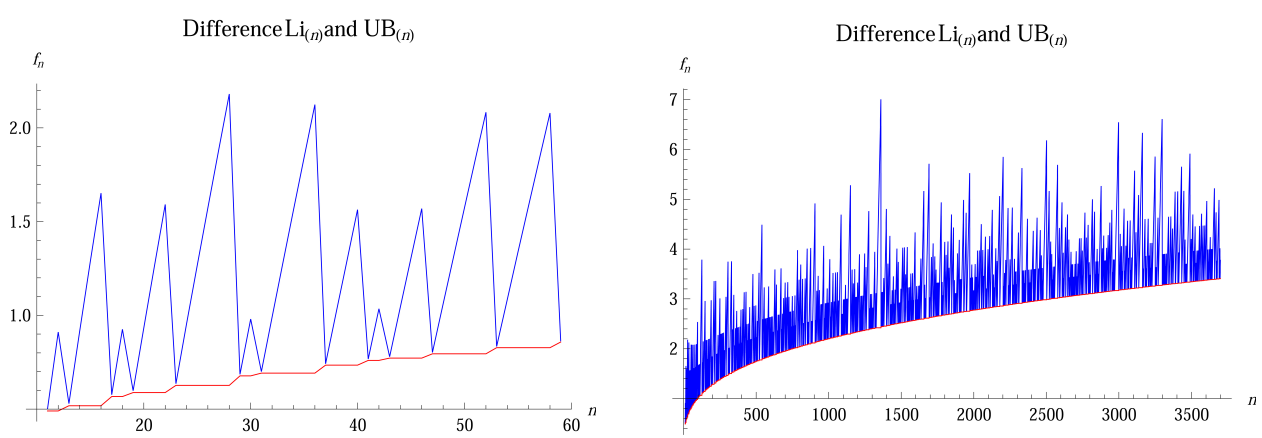

FiguRE 9. The drawings show the graph of the true difference $\left(L i_{(n)}-U B_{(n)}\right)$ (Blue), and the estimate of this value given by 5.71 (Red). The figures are drawn with respect to $n \in \mathbb{N}$ at every $n$ within the respective range.

Difference $\left(\mathrm{Li}_{(n)}-\mathrm{UB}_{(n)}\right)$
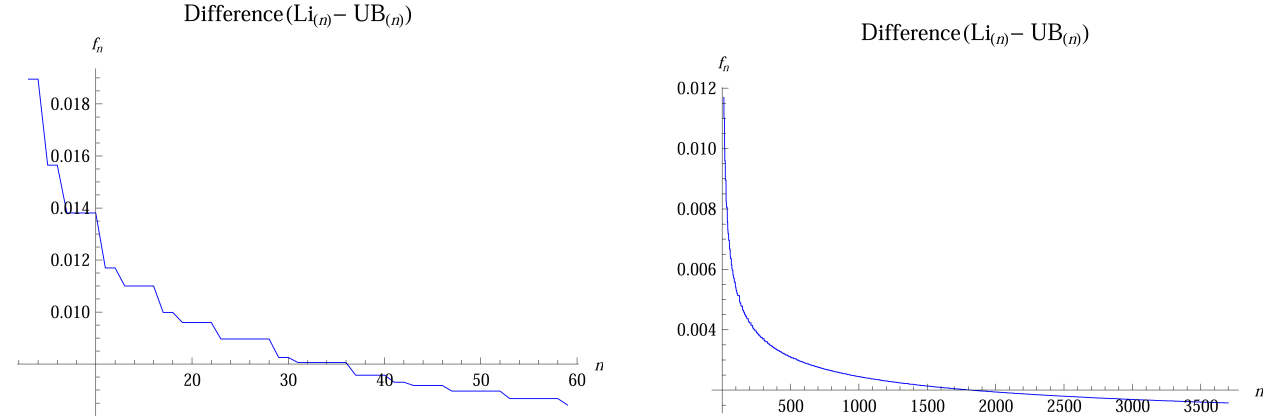

Figure 10. The drawings show the graph of the difference of $\left(L i_{(n)}-U B_{(n)}\right)$ and the estimate 5.71 , computed at the primes only. The figures are drawn with respect to $n \in \mathbb{N}$ at every $n$ within the respective range.

Remark 5.9. Due to the uneven distribution of primes, both the difference $n-p_{(n)}$ and $p_{(n)}-\log p_{(n)} \sharp$ are inherently highly oscillatory. Approximating $\log p_{(n)} \sharp$ by the difference:

$$
p_{(n)}-(\sqrt{5}-1)\left(4 \gamma^{2}-2 \gamma\right)\left(\log p_{(n)}\right) \sqrt[3]{p_{(n)}}
$$

as the upper integration limit for the $U B_{(n)}$, incorporates this effect into the estimation error of the $U B_{(n)}$. Another consequence of the application of the estimates of $\log p_{(n)} \sharp$ instead of the exact values, is that $U B_{(n)}$ also exhibits the tendency to follow the Gauss' $L i_{(n)}$. This is clearly visible over the intervals where both $\pi_{(n)}$ and $T L i_{(n)}$ tend to "sag", the estimate $U B_{(n)}$ keeps on going relatively unaffected. Please refer to the graph 18 in the Appendix. Because $U B_{(n)}$ uses the value of the greatest prime $p \in \mathbb{N} \mid p \leq n$ in the calculation of the upper limit of integration, as a result its graph continues in a straight level line across every prime gap.

The omission of the term $n-p_{(n)}$ from the estimate of the difference of $L i_{(n)}$ and $U B_{(n)}$ :

$$
\int_{\theta_{\mathcal{U}}}^{n} \frac{d t}{\log t} \gtrsim(\sqrt{5}-1)\left(4 \gamma^{2}-2 \gamma\right) \sqrt[3]{p_{(n)}}
$$


caused the results to be accurate at the primes only; since $n=p_{(n)}$ at such point. In the intermittent space, the true value of $L i_{(n)}-U B_{(n)}$ increases.

The greater the gap between the primes, the greater the difference between the estimate 5.71 and the true value of the difference $L i_{(n)}-U B_{(n)}$. Consequently, for a given prime gap, we have that the locally biggest difference occurs at every $n \in \mathbb{N} \mid n=p_{(n)}-1$.

However, by Theorem 3.6, the difference $n-p_{(n)}$ is bound to be less than the maximal gaps Supremum:

$$
\frac{n-p_{(n)}}{\log p_{(n)}}<\frac{\mathcal{G}_{\left(p_{(n)}\right)}}{\log p_{(n)}}=\frac{5}{\log 10}\left(\log _{10} p_{(n)}\right) \approx 2.17147\left(\log _{10} p_{(n)}\right)
$$

hence the relative contribution of the difference $n-p_{(n)}$ decreases as $n$ increases, consequently, gradually losing significance. In fact at $10^{15}$ the ratio:

$$
\frac{\frac{5}{\log 10}\left(\log _{10} n\right)}{\left((\sqrt{5}-1)\left(4 \gamma^{2}-2 \gamma\right) \sqrt[3]{n}\right)} \approx 0.00147809
$$

thus, the error made in estimation by omission of the term $\left(n-\theta_{\mathcal{U}}\right)$, is less than 0.15 percent at that point.

Theorem 5.22 (Infimum Of The Difference $L i_{(p)}-U B_{(p)}$ ).

In an instance when $n$ is a prime number, the Infimum bound on the difference $L i_{(p)}-U B_{(p)}$ computed at the primes $p \in \mathbb{N} \mid p \geq 11$, is given by:

$$
\mathcal{L} \mathcal{T} \mathcal{I}_{(p)}=(\sqrt{5}-1)\left(4 \gamma^{2}-2 \gamma\right) \sqrt[3]{p} \leq L i_{(p)}-U B_{(p)}
$$

In this case, the upper estimation error bound, for all $p \in \mathbb{N} \mid p \geq 11$ is given by,

$$
\begin{aligned}
& {\left[L i_{\left(p_{(n)}\right)}-\right.}\left.U B_{\left(p_{(n)}\right)}-\mathcal{L} \mathcal{T} \mathcal{I}_{\left(p_{(n)}\right)}\right] \leq \frac{1}{\left(\log (10)\left(\log \left(p_{n}\right)\right)\right)^{2}} \equiv \\
& \equiv\left[L i_{\left(p_{(n)}\right)}-U B_{\left(p_{(n)}\right)}\right] \leq \frac{1}{\left(\log (10)\left(\log \left(p_{n}\right)\right)\right)^{2}}+\mathcal{L} \mathcal{I}_{\left(p_{(n)}\right)}
\end{aligned}
$$

Similarly, the lower estimation error bound, for all $p \in \mathbb{N} \mid p \geq 263$ is given by:

$$
\begin{aligned}
\frac{1}{p_{(n)}} \leq\left[L i_{\left(p_{(n)}\right)}-U B_{\left(p_{(n)}\right)}\right. & \left.-\mathcal{L T}_{\left(p_{(n)}\right)}\right] \equiv \\
& \equiv \frac{1}{p_{(n)}}+\mathcal{L} \mathcal{T} \mathcal{I}_{\left(p_{(n)}\right)} \leq\left[L i_{\left(p_{(n)}\right)}-U B_{\left(p_{(n)}\right)}\right]
\end{aligned}
$$

Proof.

From Definition 5.18 and Theorem 5.20, the length of the interval separating the $L i_{(n)}$ and $U B_{(n)}$ is given by:

$$
\left(n-\theta_{\mathcal{U}}\right)=(\sqrt{5}-1)\left(4 \gamma^{2}-2 \gamma\right)\left(\log p_{(n)}\right) \sqrt[3]{p_{(n)}}+\left(n-p_{(n)}\right)
$$

where $p_{(n)}$ is the biggest prime $p \leq n$. When $n$ is a prime however, $\left(n-p_{(n)}\right)=0$. By the application of the PNT therefore, we may approximate the true value of the 
difference $L i_{\left(p_{(n)}\right)}-U B_{\left(p_{(n)}\right)}$ at the primes within the range:

$$
\begin{aligned}
\int_{\theta_{\mathcal{U}}}^{p_{(n)}} \frac{d t}{\log t} \sim \frac{(\sqrt{5}-1)\left(4 \gamma^{2}-2 \gamma\right)\left(\log p_{(n)}\right) \sqrt[3]{p_{(n)}}}{\log p_{(n)}} & =(\sqrt{5}-1)\left(4 \gamma^{2}-2 \gamma\right) \sqrt[3]{p_{(n)}}=\mathcal{L} \mathcal{T} \mathcal{I}_{\left(p_{(n)}\right)}
\end{aligned}
$$

$\mathcal{L T I}_{\left(p_{(n)}\right)}$ is a positive, monotone, increasing without a bound function. By Theorem 5.21, for all $n \in \mathbb{N} \mid n \geq 11$ the difference $L i_{(n)}-U B_{(n)}>0$ and diverges. The Gauss' offset integral $L i_{(n)}$ clearly increases monotonically, as well as the bound $U B_{(n)}$. Suppose that Theorem 5.22 is false, thus, in accordance with the hypothesis the difference:

$$
L i_{\left(p_{(n)}\right)}-U B_{\left(p_{(n)}\right)}-\mathcal{L} \mathcal{T} \mathcal{I}_{\left(p_{(n)}\right)}<0
$$

However, the inequality 5.75 at $p_{n}=11$ attains $\sim 0.011694$ and asymptotically tends to zero as $p_{n}$ increases unboundedly. Therefore, it is a positive decreasing sequence of real numbers. We implement therefore the Second Ratio Test. Define the test sequence:

$$
a_{\left(p_{(n)}\right)}=\left\{L i_{\left(p_{(n)}\right)}-U B_{\left(p_{(n)}\right)}-\mathcal{L} \mathcal{T} \mathcal{I}_{\left(p_{(n)}\right)}\right\}
$$

the Second Ratio Test, given by $\max \left\{\frac{a_{\left(2 p_{n}\right)}}{a_{p_{n}}}, \frac{a_{\left(\left(2 p_{n}\right)+1\right)}}{a_{p_{n}}}\right\}$ :

$$
\lim _{p_{n} \rightarrow \infty} \frac{a_{2 p_{n}}}{a_{p_{n}}} \sim \frac{\pi}{4}
$$

The test sequence tends from below asymptotically to $\sim \frac{\pi}{4}>\frac{1}{2}$. Therefore, by the definition of the Second Ratio Test we conclude that, the series formed by the terms of 5.76 diverges as $p_{n}$ increases unboundedly. Consequently, we have a contradiction to the initial hypothesis. Therefore, for all $p \in \mathbb{N} \mid p \geq 11$ the relation is valid:

$$
\mathcal{L} \mathcal{I}_{\left(p_{(n)}\right)} \leq L i_{\left(p_{(n)}\right)}-U B_{\left(p_{(n)}\right)}
$$

Suppose now that $\forall n \in \mathbb{N} \mid n \geq 347$ the following inequality is false:

$$
\left[L i_{\left(p_{(n)}\right)}-U B_{\left(p_{(n)}\right)}-\mathcal{L} \mathcal{T} \mathcal{I}_{\left(p_{(n)}\right)}\right]^{-1}-p_{(n)}<0
$$

However, the relation at $p_{n}=347$ attains $\sim-60.9818$ and diverges with a rate of divergence $\propto k p_{n} \mid$ for $k \sim 1$ for larger $p_{n}$. Therefore, for all $p \in \mathbb{N} \mid p \geq 263$ define a positive valued test sequence and implement the Cauchy Root test:

$$
\lim _{n \rightarrow \infty} \sqrt[n]{a_{n}}=\lim _{n \rightarrow \infty} \sqrt[n]{\left(p_{(n)}-\left[L i_{\left(p_{(n)}\right)}-U B_{\left(p_{(n)}\right)}-\mathcal{L} \mathcal{T} \mathcal{I}_{\left(p_{(n)}\right)}\right]^{-1}\right)} \rightarrow 1
$$

The Root Test, at $p_{n}=347$ attains $\sim 1.01191647$ and converges asymptotically, strictly from above to 1 . Consequently, by the definition of the Cauchy's Root Test, the series formed from the terms of the sequence diverges. Hence, we have a contradiction to the initial hypothesis. Consequently, $\forall n \in \mathbb{N} \mid n \geq 347$ the inequality is valid:

$$
\left[L i_{\left(p_{(n)}\right)}-U B_{\left(p_{(n)}\right)}-\mathcal{L} \mathcal{T} \mathcal{I}_{\left(p_{(n)}\right)}\right]^{-1}<p_{(n)}
$$


Suppose now in turn, that $\forall n \in \mathbb{N} \mid n \geq 11$ the following inequality is false:

$$
\left[L i_{\left(p_{(n)}\right)}-U B_{\left(p_{(n)}\right)}-\mathcal{L} \mathcal{T} \mathcal{I}_{\left(p_{(n)}\right)}\right]^{-1}-\left(\log (10)\left(\log \left(p_{n}\right)\right)\right)^{2}>0
$$

However, inequality 5.80 , at $p_{n}=11$ attains approximately 55.0282 and diverges. Therefore, define a positive valued test sequence and apply the Cauchy's Root Test:

$$
\begin{aligned}
& \lim _{n \rightarrow \infty} \sqrt[n]{a_{n}}= \\
& \lim _{n \rightarrow \infty} \sqrt[n]{\left\{\left[L i_{\left(p_{(n)}\right)}-U B_{\left(p_{(n)}\right)}-\mathcal{L} \mathcal{T I}_{\left(p_{(n)}\right)}\right]^{-1}-\left(\log (10)\left(\log \left(p_{n}\right)\right)\right)^{2}\right\}} \rightarrow 1
\end{aligned}
$$

At $p_{n}=11$ the Root Test attains approximately 1.43958 and converges asymptotically, strictly from above to 1 . Consequently, by the definition of the Cauchy's Root Test, the series formed from the terms of the sequence diverges. Therefore, we have a contradiction to the initial hypothesis. This implies that $\forall n \in \mathbb{N} \mid n \geq 11$ the inequality is valid:

$$
\left[L i_{\left(p_{(n)}\right)}-U B_{\left(p_{(n)}\right)}-\mathcal{L} \mathcal{T} \mathcal{I}_{\left(p_{(n)}\right)}\right]^{-1}>\left(\log (10)\left(\log \left(p_{n}\right)\right)\right)^{2}
$$

Hence, from the Inequalities 5.77, 5.79 and 5.81 for all $p \in \mathbb{N} \mid p \geq 11$ we have:

$$
\mathcal{L} \mathcal{T} \mathcal{I}_{(p)}=(\sqrt{5}-1)\left(4 \gamma^{2}-2 \gamma\right) \sqrt[3]{p} \leq L i_{(p)}-U B_{(p)}
$$

as well as,

$$
\begin{aligned}
& {\left[L i_{\left(p_{(n)}\right)}-\right.}\left.U B_{\left(p_{(n)}\right)}-\mathcal{L} \mathcal{T} \mathcal{I}_{\left(p_{(n)}\right)}\right] \leq \frac{1}{\left(\log (10)\left(\log \left(p_{n}\right)\right)\right)^{2}} \equiv \\
& \equiv\left[L i_{\left(p_{(n)}\right)}-U B_{\left(p_{(n)}\right)}\right] \leq \frac{1}{\left(\log (10)\left(\log \left(p_{n}\right)\right)\right)^{2}}+\mathcal{L} \mathcal{T} \mathcal{I}_{\left(p_{(n)}\right)}
\end{aligned}
$$

Furthermore, for all $p \in \mathbb{N} \mid p \geq 347$ we have:

$$
\begin{aligned}
\frac{1}{p_{(n)}} \leq\left[L i_{\left(p_{(n)}\right)}-U B_{\left(p_{(n)}\right)}\right. & \left.-\mathcal{L} \mathcal{I}_{\left(p_{(n)}\right)}\right] \equiv \\
& \equiv \frac{1}{p_{(n)}}+\mathcal{L} \mathcal{T} \mathcal{I}_{\left(p_{(n)}\right)} \leq\left[L i_{\left(p_{(n)}\right)}-U B_{\left(p_{(n)}\right)}\right]
\end{aligned}
$$

A straightforward computer calculation verifies that the Inequality 5.84 holds for all $p \in \mathbb{N} \mid 263 \leq p \leq 347$ as well. Consequently, Inequality 5.84 holds for all primes $p \in \mathbb{N} \mid p \geq 263$ as stated, thus concluding the proof.

Theorem 5.22 implies that we have a very good approximation of the difference $L i_{(n)}-U B_{(n)}$ at the primes within the range, hence the least difference $L i_{(n)}-$ $U B_{(n)}$.

\subsection{Supremum of $\pi_{(n)}$ and the Skewes' $\pi_{(n)}>L i_{(n)}$ problem appraisal.}

About 1792 Carl F. Gauss postulated the PNT on the basis of empirical evidence. He thought that:

$$
\pi_{(n)}<L i_{(n)} \quad \forall n \in \mathbb{N} \mid n \geq 2
$$

Gauss' belief relied on his observations made, of the tables of primes up to $n=3,000,000$. Many of those he constructed by hand himself. His belief was 
shared by Bernhard Riemann and indeed many other mathematicians of the 19-th century. In 1914 John E. Littlewood presented the proof that:

$$
\pi_{(n)}-L i_{(n)}>\frac{k \sqrt{n}}{\log n} \log (\log (\log n))
$$

Littlewood's proof of 1914 [25], [22], depends upon the size of the $\log (\log (\log n))$ for large n. Since however the Littlewood's proof was not constructive [28], Stanley Skewes in 1933 presented a proof (assuming Riemann's hypothesis), that there exist values of $n$ such that:

$$
\pi_{(n)}>L i_{(n)} \quad \text { for } n \in \mathbb{N} \mid n<10^{10^{10^{34}}}
$$

In $1955 \mathrm{~S}$. Skewes re-appraised the problem, this time without the assumption of Riemann's hypothesis he produced a different bound:

$$
\pi_{(n)}>\operatorname{Li}(n) \quad \text { for } n \in \mathbb{N} \mid n<10^{10^{10^{10^{3}}}}
$$

This legendary bound has since been lowered very significantly, however, it still remains out of reach of direct verification [2]. The theory of the tailored integral developed up to this point permits us to attack Skewes' problem and to prove conclusively that $L i_{(n)}>\pi_{(n)} \quad \forall n \in \mathbb{N} \mid n \geq 11$.

Theorem 5.23 (The Supremum Bound Of Estimation Of $\pi_{(n)}$ ).

The Tailored Integral is less or at most equal in value to the estimate $U B_{(n)}$ :

$$
U B_{(n)}-T L i_{(n)}=\int_{\theta_{(n)}}^{\theta_{\mathcal{U}}} \frac{d t}{\log t} \quad \geq 0 \quad \forall n \in \mathbb{N} \mid n \geq 11
$$

Further, the tailored logarithmic integral $T L i_{(n)}$ constitutes the Supremum estimation bound of the prime counting function $\pi_{(n)}$ :

$$
\pi_{(n)}<\int_{2}^{\theta_{(n)}} \frac{d t}{\log t} \leq \int_{2}^{\theta_{\mathcal{U}}} \frac{d t}{\log t}<\int_{2}^{n} \frac{d t}{\log t} \quad \forall n \in \mathbb{N} \mid n \geq 43
$$

consequently,

$$
L i_{(n)}=\int_{2}^{n} \frac{d t}{\log t}>\pi_{(n)} \quad \forall n \in \mathbb{N} \mid n \geq 11
$$

Proof.

By Theorem 5.21 we have that:

$$
L i_{(n)}=\int_{2}^{n} \frac{d t}{\log t}>U B_{(n)}=\int_{2}^{\theta_{\mathcal{U}}} \frac{d t}{\log t} \quad \forall n \in \mathbb{N} \mid n \geq 11
$$

where the difference in values taken by the estimate of the tailored logarithmic integral and the Gauss' logarithmic integral diverges as n tends to infinity:

$$
\lim _{n \rightarrow \infty}\left(L i_{(n)}-U B_{(n)}\right)=\lim _{n \rightarrow \infty}\left(\int_{\theta_{\mathcal{U}}}^{n} \frac{d t}{\log t}\right) \rightarrow \infty
$$

On the other hand, by Theorem 5.15 we have:

$$
T L i_{(n)}=\int_{2}^{\theta_{(n)}} \frac{d t}{\log t}>\pi_{(n)} \quad \forall n \in \mathbb{N} \mid n \geq 43
$$


With the estimation error increasing unboundedly as $\mathrm{n}$ tends to infinity:

$$
\begin{array}{r}
\lim _{n \rightarrow \infty}\left(T L i_{(n)}-\pi_{(n)}\right)=\lim _{n \rightarrow \infty}\left(\sum_{p_{(i+1)}=47}^{p_{(n)}}\left\{\left(\int_{\theta_{1}}^{\theta_{2}} \frac{d t}{\log t}\right)-1\right\}+\left(\int_{2}^{\theta_{43}} \frac{d t}{\log t}-\pi_{43}\right)\right) \rightarrow \infty \\
\text { where }\left(\int_{2}^{\theta_{43}} \frac{d t}{\log t}-14\right) \approx 0.002993180461560385
\end{array}
$$

Now, by Lemmas 4.5 and 4.7 , we have for all $p_{(n)} \in \mathbb{N} \mid p_{(n)} \geq 2$ where $p_{n}$ is the greatest prime number $p \in \mathbb{N} \mid p \leq n$ :

$$
\left(p_{(n)}-2 \sqrt{p_{(n)}}\right)<\log p_{(n)} \sharp<\left(p_{(n)}-(\sqrt{5}-1)\left(4 \gamma^{2}-2 \gamma\right)\left(\log p_{(n)}\right) \sqrt[3]{p_{(n)}}\right)=\theta_{\mathcal{U}}
$$

Which gives the interval containing the true value of $\theta_{(n)}=\log p_{(n)} \sharp$ :

$$
\begin{aligned}
2 \sqrt{p_{(n)}}- & (\sqrt{5}-1)\left(4 \gamma^{2}-2 \gamma\right)\left(\log p_{(n)}\right) \sqrt[3]{p_{(n)}} \\
& =\left(\frac{2}{\sqrt{p_{(n)}}\left(\log p_{(n)}\right)}-\frac{(\sqrt{5}-1)\left(4 \gamma^{2}-2 \gamma\right)}{p_{(n)}^{2 / 3}}\right) \times p_{(n)}\left(\log p_{(n)}\right)
\end{aligned}
$$

The upper endpoint of the interval 5.94 is the $\theta_{\mathcal{U}}$. In the case that the value of $\log p_{(n)} \sharp$ is located close to the upper endpoint of the interval 5.93 , this implies that $\theta_{(n)} \lesssim \theta_{\mathcal{u}}$. Consequently,

$$
U B_{(n)}-T L i_{(n)}=\int_{\theta_{(n)}}^{\theta_{\mathcal{U}}} \frac{d t}{\log t} \gtrsim 0
$$

Hence, the Infimum of the difference $U B_{(n)}-T L i_{(n)}$ is:

$$
\inf \left(U B_{(n)}-T L i_{(n)}\right)=\inf \left(\int_{\theta_{(n)}}^{\theta_{\mathcal{U}}} \frac{d t}{\log t}\right)=0 \quad \forall p_{(n)} \in \mathbb{N} \mid p_{(n)} \geq 11
$$

The maximum possible value of the difference $U B_{(n)}-T L i_{(n)}$ may be estimated by using the length of the interval 5.94 and applying the PNT:

$$
\begin{aligned}
U B_{(n)}-T L i_{(n)}= & \int_{\theta_{(n)}}^{\theta_{\mathcal{U}}} \frac{d t}{\log t} \\
& \lesssim\left(\frac{2}{\sqrt{p_{(n)}}\left(\log p_{(n)}\right)}-\frac{(\sqrt{5}-1)\left(4 \gamma^{2}-2 \gamma\right)}{p_{(n)}^{2 / 3}}\right) \times p_{(n)}
\end{aligned}
$$

Clearly, the limit of the estimated maximum value of the difference 5.95 diverges, as $\mathrm{n}$ tends to infinity. Hence we have that:

$$
\begin{aligned}
& \text { 96) } 0 \leq U B_{(n)}-T L i_{(n)}=\int_{\theta_{(n)}}^{\theta_{\mathcal{U}}} \frac{d t}{\log t} \\
& \leq\left(\frac{2}{\sqrt{p_{(n)}}\left(\log p_{(n)}\right)}-\frac{(\sqrt{5}-1)\left(4 \gamma^{2}-2 \gamma\right)}{p_{(n)}^{2 / 3}}\right) \times p_{(n)} \quad \forall p_{(n)} \in \mathbb{N} \mid p_{(n)} \geq 11
\end{aligned}
$$


as well as by Theorem 5.15 we have:

$$
T L i_{(n)}-\pi_{(n)}=\int_{2}^{\theta_{(n)}} \frac{d t}{\log t}-\pi_{(n)}>0 \text { for } n \in \mathbb{N} \mid n \geq 43
$$

Since $U B_{(n)}-T L i_{(n)} \geq 0$ for all $p_{(n)} \in \mathbb{N} \mid p_{(n)} \geq 43, T L i_{(n)}-\pi_{(n)}>0$ for all $n \in \mathbb{N} \mid n \geq 43$, this implies that $U B_{(n)}-\pi_{(n)}>0$ for all $n \in \mathbb{N} \mid n \geq 43$. Direct computation shows that $U B_{(n)}-\pi_{(n)}>0$ for all $n \in \mathbb{N} \mid 23 \leq n \leq 43$. Please refer to Table 4 below. Consequently, from 5.89, 5.91 and 5.96 we have that:

$$
\pi_{(n)}<\int_{2}^{\theta_{(n)}} \frac{d t}{\log t} \leq \int_{2}^{\theta_{\mathcal{U}}} \frac{d t}{\log t}<\int_{2}^{n} \frac{d t}{\log t} \text { for } n \in \mathbb{N} \mid n \geq 43
$$

This shows that $T L i_{(n)}$ constitutes the Supremum estimation bound of the prime counting function $\pi_{(n)}$ for all $n \in \mathbb{N} \mid n \geq 43$. Direct computation confirms that the difference $U B_{(n)}-T L i_{(n)}>0$ holds for all $n \in \mathbb{N} \mid 11 \leq n \leq 43$. Please refer to Fig. 11. Therefore, Theorem 5.23 holds as stated, concluding the proof.

TABLE 4. Low range difference $U B_{(n)}-\pi_{(n)}$

\begin{tabular}{|c|c|c|c|}
\hline$n$ & $U B_{(n)}-\pi_{(n)}$ & $n$ & $U B_{(n)}-\pi_{(n)}$ \\
\hline 11 & 0.0440603 & 29 & 0.9967 \\
\hline 13 & -0.177776 & 31 & 0.569752 \\
\hline 17 & 0.254689 & 37 & 1.23064 \\
\hline 19 & -0.0741603 & 41 & 1.29738 \\
\hline 23 & 0.202729 & 43 & 0.820459 \\
\hline
\end{tabular}

Corollary 5.24 (Littlewood - Skewes' problem).

Theorem 5.23 implies that we have a strong contradiction to the statement, that infinitely often:

$$
\pi_{(n)}-L i_{(n)}>0
$$

Necessarily, this implies that the Littlewood's theorem of 1914 and hence the relation 5.97 above are both false, disproving them for every $n \in \mathbb{N} \mid n \geq 11$. Since the relation 5.97 is obviously false within the range $n \in \mathbb{N} \mid 2 \leq n \leq 11$, consequently this implies that the Littlewood's theorem of 1914 is false for every $n \in \mathbb{N}$.

Theorem 5.23 implies that Carl F. Gauss' belief, shared by Bernhard Riemann and indeed many other mathematicians of the 19-th century, was correct thereby proving their historical guess.

\subsection{Estimation error bounds on the difference $T L i_{(n)}-\pi_{(n)}$.}

Because the Infimum and Supremum estimation error bounds are inherently difficult to compute for large $n \in \mathbb{N}$, this section presents alternative lower and upper estimation error bounds.

Theorem 5.25 (Lower Estimation Error Bound).

The error of estimation of the prime counting function $\pi_{(m)}$ by the application of the tailored logarithmic integral $T L i_{(m)}$, for all $m \in \mathbb{N} \mid m \geq 11$ is bounded below 

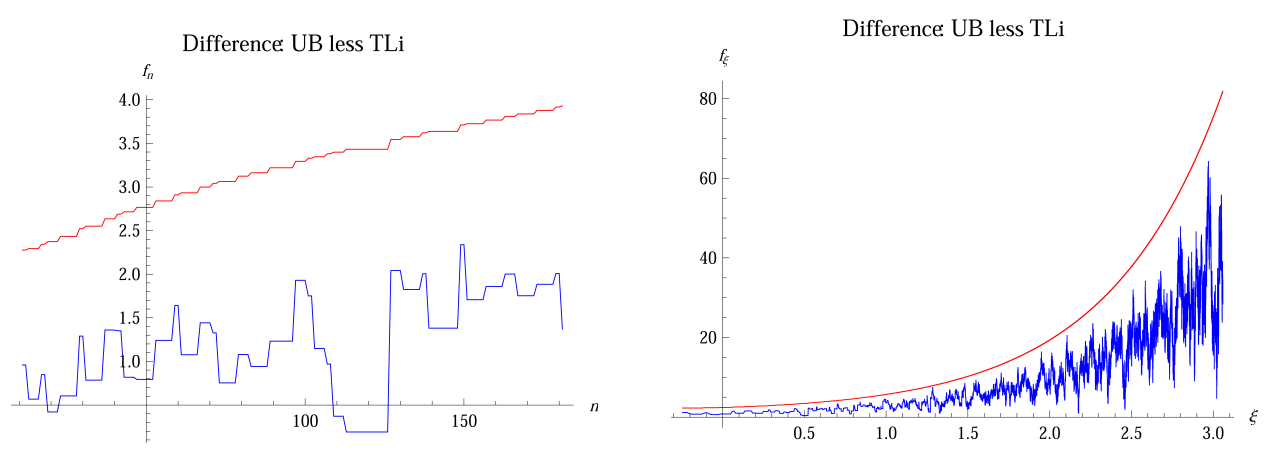

FIGURE 11. The drawings show the graph of the true difference $\left(U B_{(n)}-T L i_{(n)}\right)$ (blue), and the estimate of the maximal value of the difference given by 5.95 (red). The L.H. figure is drawn at every $n \in \mathbb{N} \mid 11 \leq n \leq 181$, while the R.H. figure drawn w.r.t. $\xi$, at every $n \in \mathbb{N} \mid 11 \leq n \leq 400009$.

by:

$$
\begin{aligned}
& T L i_{(m)}-\pi_{(m)} \geq \\
& \mathcal{L E B}_{(m)}=\frac{\mathcal{A}(\log 10)}{2 \gamma\left(\log _{10} m\right)^{(\sqrt{3}-1)}}\left(\frac{\sqrt{m}}{\left(\log _{10} m\right)^{2}}+\frac{5}{\log 10}\left(\log _{10} m\right)-6\right)-(\exp (2))
\end{aligned}
$$

Where $\gamma$ is the Euler-Mascheroni constant and $\mathcal{A}$ is the Glaisher-Kinkelin constant given by definition 1.5.

Proof.

Evidently, $\pi_{(m)}$ defines a weakly monotone, divergent function. By Theorem 5.15 the estimation error of the tailored logarithmic integral $T L i_{(m)}$ defines a monotone divergent sequence. Also, the lower estimation error bound $\mathcal{L E B}_{(m)}$ clearly defines a monotone divergent sequence. Suppose that Theorem 5.25 is false for $m \in \mathbb{N} \mid m \geq$ 1000000007 , then it has to be true that:

$$
T L i_{(m)}<\left(\mathcal{L E B}_{(m)}+\pi_{(m)}\right)
$$

Which is equivalent to say,

$$
\exp \left(T L i_{(m)}\right)-\exp \left(\mathcal{L E B}_{(m)}+\pi_{(m)}\right)<0
$$

However, at $m=1000000007$ the difference 5.100 attains $\sim 9.9890903 * 10^{22082891}$ and rapidly diverges as $m$ increases unboundedly. Therefore, the difference 5.100 generates positive numerical sequence in $\mathbb{R}$. Thus, we implement the Cauchy's Root Test ${ }^{1}$ :

$$
\begin{aligned}
C=\lim _{n \rightarrow \infty} \sqrt[n]{\left|a_{n}\right|}=\lim _{n \rightarrow \infty} \sqrt[n]{\exp \left(T L i_{(m)}\right)-\exp \left(\mathcal{L E} \mathcal{B}_{(m)}+\pi_{(m)}\right)} \rightarrow \\
\rightarrow \exp (1) \approx 2.718281828>1
\end{aligned}
$$

The test at $m=1000000007$ attains $\sim 2.7182926769186047$ and converges to $\sim \exp (1)$ strictly from above. By the definition of the Cauchy's Root Test this

\footnotetext{
${ }^{1}$ The degree $n$ of the root pertains to the prime number $p_{n}$ being the $\mathrm{n}$-th prime number, the largest one that satisfies the relation $p_{n} \leq m$.
} 
implies that a series formed by the terms of the Inequality 5.100 necessarily diverges. Consequently, the difference:

$$
\begin{aligned}
& T L i_{(m)} \\
& -\left(\frac{\mathcal{A}(\log 10)}{2 \gamma\left(\log _{10} m\right)^{(\sqrt{3}-1)}}\left(\frac{\sqrt{m}}{\left(\log _{10} m\right)^{2}}+\frac{5}{\log 10}\left(\log _{10} m\right)-6\right)-(\exp (2))+\pi_{(m)}\right)>0
\end{aligned}
$$

for all $m \in \mathbb{N} \mid m \geq 1000000007$. This implies that we have a contradiction to the hypothesis. Direct computer calculation confirms that Theorem 5.25 also holds within the range for all $m \in \mathbb{N} \mid 11 \leq m \leq 1000000007$. The pertinent data had been rendered in graphical form, please refer to Fig. 13 to 17 in the Appendix. Therefore, Theorem 5.25 holds for all $m \in \mathbb{N} \mid m \geq 11$; thus concluding the proof.

Theorem 5.26 (Upper Estimation Error Bound).

The error of estimation of the prime counting function $\pi_{(m)}$ by the application of the tailored logarithmic integral $T L i_{(m)}$, for all $m \in \mathbb{N} \mid m \geq 3$ is bounded above by:

$$
\begin{aligned}
& T L i_{(m)}-\pi_{(m)} \\
\leq & \mathcal{U E B} \mathcal{B}_{(m)}=\mathcal{T C}\left(\frac{\sqrt{m}}{\left(\log _{10} m\right)^{(\sqrt{2}+1)}}+\frac{5\left(\log _{10} m\right)^{2}}{(\log 10)\left(\log _{10} m\right)^{(\sqrt{2}+1)}}+\frac{7}{\left(\log _{10} m\right)^{\sqrt{2}}}\right)
\end{aligned}
$$

Where $\mathcal{T C}$ is given by the Definition 1.6.

Proof.

Evidently, $\pi_{(m)}$ defines a weakly monotone, divergent function. By Theorem 5.15 the estimation error of the tailored logarithmic integral $T L i_{(m)}$ defines a monotone divergent sequence. Also, the upper estimation error bound $\mathcal{U} \mathcal{E B}_{(m)}$ clearly defines a monotone divergent sequence. Suppose that Theorem 5.26 is false for $m \in \mathbb{N} \mid m \geq$ 1000000007 , then it has to be true that:

$$
T L i_{(m)}>\mathcal{U E B} \mathcal{B}_{(m)}+\pi_{(m)}
$$

Which is equivalent to say,

$$
\exp \left(\mathcal{U E} \mathcal{B}_{(m)}+\pi_{(m)}\right)-\exp \left(T L i_{(m)}\right)<0
$$

However, at $m=1000000007$ the difference 5.103 attains $\sim 3.81666351 * 10^{29156538}$ and rapidly diverges as $m$ increases unboundedly. Therefore, the difference 5.103 generates positive numerical sequence in $\mathbb{R}$. Thus, we implement the Cauchy's Root Test ${ }^{2}$ :

$$
\begin{aligned}
& C=\lim _{n \rightarrow \infty} \sqrt[n]{\left|a_{n}\right|} \\
&=\lim _{n \rightarrow \infty} \sqrt[n]{\exp \left(\mathcal{U E \mathcal { B }}(m)+\pi_{(m)}\right)-\exp \left(T L i_{(m)}\right)} \rightarrow \\
& \rightarrow \exp (1) \approx 2.718281828>1
\end{aligned}
$$

The test at $m=1000000007$ attains $\sim 2.71829300286192$ and converges to $\sim$ $\exp (1)$ strictly from above. By the definition of the Cauchy's Root Test this implies that a series formed by the terms of the Inequality 5.103 necessarily diverges.

\footnotetext{
${ }^{2}$ The degree $n$ of the root pertains to the prime number $p_{n}$ being the $\mathrm{n}$-th prime number, the largest one that satisfies the relation $p_{n} \leq m$.
} 
Consequently, the difference:

$$
\begin{aligned}
\left(\mathcal{T C}\left(\frac{\sqrt{m}}{\left(\log _{10} m\right)^{(\sqrt{2}+1)}}+\frac{5\left(\log _{10} m\right)^{2}}{(\log 10)\left(\log _{10} m\right)^{(\sqrt{2}+1)}}+\frac{7}{\left(\log _{10} m\right)^{\sqrt{2}}}\right)+\pi_{(m)}\right) \\
-T L i_{(m)}>0
\end{aligned}
$$

for all $m \in \mathbb{N} \mid m \geq 1000000007$. This implies that we have a contradiction to the hypothesis. Direct computation $\forall m \in \mathbb{N} \mid 3 \leq m \leq 1000000007$ verifies that Theorem 5.26 also holds within this range. Pertinent data had been rendered in graphical form, please refer to Fig. 13 to 17 in the Appendix. Therefore, Theorem 5.26 holds for all $m \in \mathbb{N} \mid m \geq 3$, concluding the proof.

Remark 5.10. Both the lower and upper estimation error bound follow the $T L i_{(n)}$ estimation error curve very closely. This situation extends over a prolonged interval. Please refer to Table 6 in the Appendix for a listing of the local minima.

\section{Gauss' logarithmic integral $L i_{(n)}$ and Riemann's hypothesis}

This section develops the mathematical basis, to link unambiguously the tailored integral theory with the Gauss' logarithmic integral error term.

\subsection{Divergence of the estimation error of $L i_{(n)}$.}

Theorem 6.1 (Divergence of the estimation error of $L i_{(n)}$ ).

The estimation error of the Gauss' logarithmic integral Li $i_{(n)}$ diverges as $n$ tends to infinity:

$$
\lim _{n \rightarrow \infty}\left(L i_{(n)}-\pi_{(n)}\right) \rightarrow \infty
$$

Proof.

By Theorems: $5.15,5.21$ and 5.23 we have that:

$$
\pi_{(n)}<\int_{2}^{\theta_{(n)}} \frac{d t}{\log t} \leq \int_{2}^{\theta_{\mathcal{U}}} \frac{d t}{\log t}<\int_{2}^{n} \frac{d t}{\log t} \text { for } n \in \mathbb{N} \mid n \geq 43
$$

and clearly:

$$
\pi_{(n)}<L i_{(n)} \quad \forall n \in \mathbb{N} \mid 11 \leq n \leq 43
$$

The estimation error of the tailored integral $T L i_{(n)}$ by Theorem 5.15 diverges:

$$
\begin{array}{r}
\lim _{n \rightarrow \infty}\left(T L i_{(n)}-\pi_{(n)}\right)=\lim _{p_{(n)} \rightarrow \infty}\left(\sum_{p_{(i+1)}=5}^{p_{(n)}}\left\{\left(\int_{\theta_{1}}^{\theta_{2}} \frac{d t}{\log t}\right)-1\right\}+\left(\int_{2}^{\theta_{3}} \frac{d t}{\log t}-\pi_{3}\right)\right) \rightarrow \infty \\
\text { where } \quad\left(\int_{2}^{\theta_{3}} \frac{d t}{\log t}-\pi_{3}\right) \approx-2.3266013098834977
\end{array}
$$

By Theorem 5.21 the difference in values between the estimate of the tailored integral and the Gauss' logarithmic integral diverges:

$$
\lim _{n \rightarrow \infty}\left(L i_{(n)}-U B_{(n)}\right)=\lim _{n \rightarrow \infty}\left(\int_{\theta_{\mathcal{U}}}^{n} \frac{d t}{\log t}\right) \rightarrow \infty
$$


Consequently therefore:

$$
\lim _{n \rightarrow \infty}\left(L i_{(n)}-\pi_{(n)}\right) \rightarrow \infty
$$

Thus concluding the proof of Theorem 6.1.

6.2. $L i_{(n)}$ estimation error bounds.
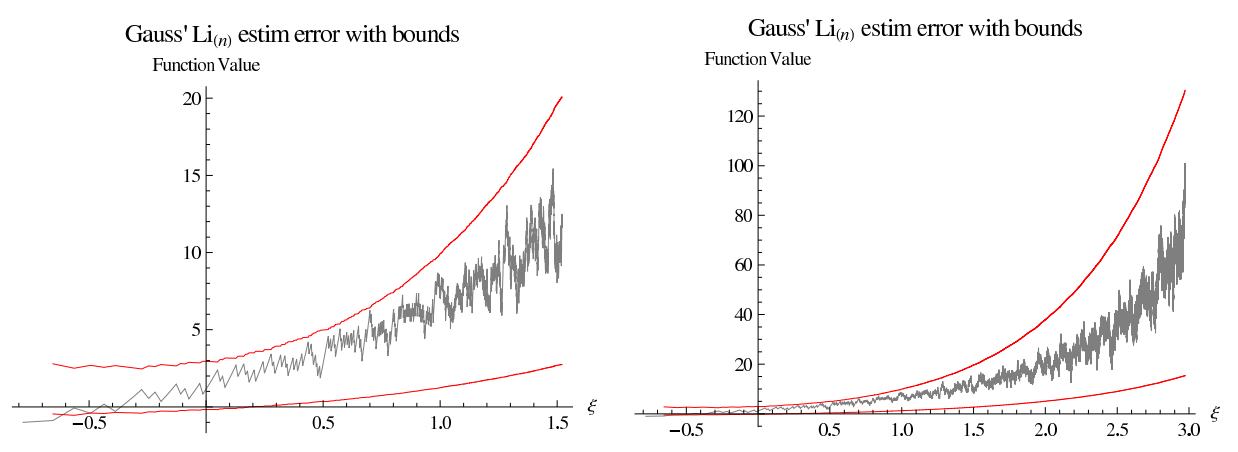

FiguRE 12. The figures show the estimation error $L i_{n}-\pi_{n}$ (gray) and the upper and lower bounds 6.10 and 6.6 (red), the figure is drawn with respect to $\xi$ at every $n \in \mathbb{N} \mid 11 \leq n \leq 3000$ in left figure and $n \in \mathbb{N} \mid 11 \leq n \leq 300000$ in right figure.

The estimation error of the tailored logarithmic integral $T L i_{(n)}$ produces virtually oscillation free curve which by Theorem 5.15 diverges:

$$
\lim _{n \rightarrow \infty}\left(T L i_{(n)}-\pi_{(n)}\right) \rightarrow \infty
$$

The smooth characteristic of the curve makes it possible to establish the estimation error bounds for the $L i_{(n)}$ by converting the estimation error bounds of the tailored logarithmic integral $T L i_{(n)}-\pi_{(n)}$ to the upper and lower bounds for the estimation error of the logarithmic integral $L i_{(n)}-\pi_{(n)}$, by the use of a specific multiplier. The upper bound implements the multiplier:

Definition 6.2. $M_{1}=\frac{2 \gamma}{\mathcal{T C}}\left(\log _{10} m\right)^{\sqrt{2}} \quad$ for $m \in \mathbb{N}$

The lower bound applies the multiplier:

Definition 6.3. $M_{2}=\frac{\left(\log _{10} m\right)^{(\sqrt{3}-1)}}{\mathcal{A}(\log 10)}$ for $m \in \mathbb{N}$

Where $\mathcal{T C}$ and $\mathcal{A}$ are given by the definitions: 1.6 and 1.5 respectively, and $\gamma$ is the Euler-Mascheroni constant gamma.

Theorem 6.4 (Lower $L i_{(m)}$ Estimation Error Bound).

For any $m \in \mathbb{N} \mid m \geq 11$, where $p_{n}$ is the $n$-th prime, the largest one satisfying the relation: $p_{n} \leq m$, the following relation holds:

$$
M_{2}\left(T L i_{(m)}-\pi_{(m)}\right)=\left(\frac{\left(\log _{10} m\right)^{(\sqrt{3}-1)}}{\mathcal{A}(\log 10)}\right)\left(T L i_{(m)}-\pi_{(m)}\right) \leq\left(L i_{(m)}-\pi_{(m)}\right)
$$

Where $\mathcal{A}$ is given by the definition 1.5. 
Proof.

The estimation error of $T L i_{(m)}$ as given by Theorem 5.15, and the estimation error of $L i_{(m)}$ as given by Theorem 6.1, both diverge as $m$ tends to infinity. Due to the fact that the $T L i_{(m)}$ estimation error increases only at the primes and remains constant between them, consequently, for all $m \in \mathbb{N} \mid m \geq 3$ it defines a weakly monotone, divergent function. Clearly, $\pi_{(m)}$ defines a weakly monotone, divergent function as well. Necessarily therefore,

$$
\left(\frac{\left(\log _{10} m\right)^{(\sqrt{3}-1)}}{\mathcal{A}(\log 10)}\right)\left(T L i_{(m)}-\pi_{(m)}\right)+\pi_{(m)}
$$

defines a monotone, divergent function. The Gauss' logarithmic integral $L i_{(m)}$ clearly is strictly monotone, divergent function. Suppose therefore, that Theorem 6.4 is false. Hence in accordance with the hypothesis we have that:

$$
\left(\frac{\left(\log _{10} m\right)^{(\sqrt{3}-1)}}{\mathcal{A}(\log 10)}\right)\left(T L i_{(m)}-\pi_{(m)}\right)+\pi_{(m)}>L i_{(m)}
$$

The difference $L i_{(m)}-\pi_{m}$ clearly is highly oscillatory, which obviously applies equally well to the difference $L i_{(m)}-T L i_{(m)}$. Therefore to smooth out the characteristics of the difference of the terms of Inequality 6.7 we take the exponential:

$$
0>\exp \left(L i_{(m)}\right)-\exp \left(\left(\frac{\left(\log _{10} m\right)^{(\sqrt{3}-1)}}{\mathcal{A}(\log 10)}\right)\left(T L i_{(m)}-\pi_{(m)}\right)+\pi_{(m)}\right)
$$

However at $p_{n}=11$, the difference attains $\sim 153.504313$ and rapidly diverges. Since the difference produces positive numerical output, we apply the Cauchy's Root Test ${ }^{3}$ :

$$
\begin{aligned}
& C=\lim _{n \rightarrow \infty} \sqrt[n]{\left|a_{n}\right|}= \\
& \lim _{n \rightarrow \infty} \sqrt[n]{\exp \left(L i_{(m)}\right)-\exp \left(\left(\frac{\left(\log _{10} m\right)^{(\sqrt{3}-1)}}{\mathcal{A}(\log 10)}\right)\left(T L i_{(m)}-\pi_{(m)}\right)+\pi_{(m)}\right)} \rightarrow \\
& \rightarrow \exp (1) \approx 2.718281828>1
\end{aligned}
$$

The test at $p_{n}=47$ attains $\sim 2.79234$ and converges strictly from above to $\sim$ $\exp (1)$. By the definition of the Cauchy's Root Test this implies that a series formed by the terms of the Inequality 6.8 necessarily diverges. Consequently, the difference:

$$
0<L i_{(m)}-\left(\left(\frac{\left(\log _{10} m\right)^{(\sqrt{3}-1)}}{\mathcal{A}(\log 10)}\right)\left(T L i_{(m)}-\pi_{(m)}\right)+\pi_{(m)}\right)
$$

for all $m \in \mathbb{N} \mid m \geq 47$. This implies that we have a contradiction to the hypothesis. Computer calculation confirms that Theorem 6.4 also holds within the range for all $m \in \mathbb{N} \mid 11 \leq m \leq 47$. Therefore, Theorem 6.4 holds for all $m \in \mathbb{N} \mid m \geq 11$; thus concluding the proof.

\footnotetext{
${ }^{3}$ The degree $n$ of the root pertains to the prime number $p_{n}$ being the $\mathrm{n}$-th prime number, the largest one that satisfies the relation $p_{n} \leq m$.
} 
Theorem 6.5 (Upper $L i_{(n)}$ Estimation Error Bound).

For any $m \in \mathbb{N} \mid m \geq 3$, where $p_{n}$ is the $n$-th prime, the largest one satisfying the relation: $p_{n} \leq m$, the following relation holds:

$$
\frac{2 \gamma}{\mathcal{T C}}\left(\log _{10} m\right)^{\sqrt{2}}\left(T L i_{(m)}-\pi_{(m)}\right)+3.5 \geq\left(L i_{(m)}-\pi_{(m)}\right)
$$

Where $\mathcal{T C}$ is given by the definition 1.6 .

Proof.

The estimation error of $T L i_{(m)}$ as given by Theorem 5.15, and the estimation error of $L i_{(m)}$ as given by Theorem 6.1, both diverge as $m$ tends to infinity. Due to the fact that the $T L i_{(m)}$ estimation error increases only at the primes and remains constant between them, consequently, for all $m \in \mathbb{N} \mid m \geq 3$ it defines a weakly monotone, increasing without bound function. Clearly, $\pi_{(m)}$ defines a weakly monotone, divergent function as well, as is $\left(\log _{10} m\right)^{\sqrt{2}}$ function. Necessarily therefore,

$$
\frac{2 \gamma}{\mathcal{T C}}\left(\log _{10} m\right)^{\sqrt{2}}\left(T L i_{(m)}-\pi_{(m)}\right)+3.5+\pi_{(m)}
$$

defines a monotone, divergent function. The Gauss' logarithmic integral $L i_{(m)}$ clearly is monotone and divergent function. Suppose therefore, that Theorem 6.5 is false. Hence in accordance with the hypothesis we have that:

$$
\frac{2 \gamma}{\mathcal{T C}}\left(\log _{10} m\right)^{\sqrt{2}}\left(T L i_{(m)}-\pi_{(m)}\right)+3.5+\pi_{(m)}<L i_{(m)}
$$

The difference $L i_{(m)}-\pi_{m}$ clearly is highly oscillatory, which obviously applies equally well to the difference $L i_{(m)}-T L i_{(m)}$. Therefore to smooth out the characteristics of the difference of the terms of Inequality 6.11 we take the exponential:

$$
\exp \left(\frac{2 \gamma}{\mathcal{T C}}\left(\log _{10} m\right)^{\sqrt{2}}\left(T L i_{(m)}-\pi_{(m)}\right)+3.5+\pi_{(m)}\right)-\exp \left(L i_{(m)}\right)<0
$$

However at $p_{n}=541$, the difference attains $\sim 3.57932 \times 10^{47}$ and rapidly diverges. Since the difference produces positive numerical output, we apply the Cauchy's Root Test ${ }^{4}$ :

$$
\begin{array}{r}
C=\lim _{n \rightarrow \infty} \sqrt[n]{\left|a_{n}\right|}= \\
\begin{array}{r}
\lim _{n \rightarrow \infty} \sqrt[n]{\exp \left(\frac{2 \gamma}{\mathcal{T C}}\left(\log _{10} m\right)^{\sqrt{2}}\left(T L i_{(m)}-\pi_{(m)}\right)+3.5+\pi_{(m)}\right)-\exp \left(L i_{(m)}\right)} \rightarrow \\
\rightarrow \exp (1) \approx 2.718281828>1
\end{array}
\end{array}
$$

The test at $p_{n}=541$ attains $\sim 2.95685$ and converges strictly from above to $\sim \exp (1)$. By the definition of the Cauchy's Root Test this implies that a series formed by the terms of the Inequality 6.12 necessarily diverges. Consequently, the difference:

$$
\left(\frac{2 \gamma}{\mathcal{T C}}\left(\log _{10} m\right)^{\sqrt{2}}\left(T L i_{(m)}-\pi_{(m)}\right)+3.5+\pi_{(m)}\right)-L i_{(m)}>0
$$

for all $m \in \mathbb{N} \mid m \geq 541$. This implies that we have a contradiction to the hypothesis. Direct computation $\forall m \in \mathbb{N} \mid 3 \leq m \leq 541$ verifies that Theorem 6.5 also holds

\footnotetext{
${ }^{4}$ The degree $n$ of the root pertains to the prime number $p_{n}$ being the $\mathrm{n}$-th prime number, the largest one that satisfies the relation $p_{n} \leq m$.
} 
within this range. Therefore Theorem 6.5 holds for all $m \in \mathbb{N} \mid m \geq 3$, concluding the proof.

Remark 6.1. Determining the $L i_{(n)}$ estimation error bounds by the application of $T L i_{(n)}$ estimation error curve is computationally very inefficient process, applicable to a relatively small values of $n \in \mathbb{N}$ only, a different more efficient method will be presented shortly.

\subsection{Primary estimation error bound of $L i_{(n)}$.}

The equation arising in estimation of the true value of $\pi_{(n)}$ by the application of the logarithmic integral

$$
\pi_{(n)}=L i_{(n)}+\mathcal{O}(\sqrt{n} \log (n))
$$

has been shown in 1901 by Niels F. Helge von Koch, to be equivalent to the Riemann's Hypothesis. The size of the estimation error term which depends on the gaps between primes, is intimately connected with the location of the zeroes of the Riemann zeta function.

Theorem 6.6 (Primary Lower Bound Of $L i_{(n)}$ Estimation Error).

For any $n \in \mathbb{N} \mid n \geq 53$, the error made in estimation of the true value of $\pi_{(n)}$ by the application of the logarithmic integral is bounded below by:

$$
\operatorname{Gauss}_{\mathcal{L}} \mathcal{L B}_{(n)}=\sum_{p \leq n} \frac{1}{p} \leq\left(L i_{(n)}-\pi_{(n)}\right)
$$

Proof. By Theorems: 5.15, 5.21 and 5.23 we have that:

$$
\pi_{(n)}<\int_{2}^{\theta_{(n)}} \frac{d t}{\log t} \leq \int_{2}^{\theta_{\mathcal{U}}} \frac{d t}{\log t}<\int_{2}^{n} \frac{d t}{\log t} \text { for } n \in \mathbb{N} \mid n \geq 43
$$

The estimation error of the tailored integral $T L i_{(n)}$ by Theorem 5.15 diverges:

$$
\begin{array}{r}
\lim _{n \rightarrow \infty}\left(T L i_{(n)}-\pi_{(n)}\right)=\lim _{p_{(n)} \rightarrow \infty}\left(\sum_{p_{(i+1)}=5}^{p_{(n)}}\left\{\left(\int_{\theta_{1}}^{\theta_{2}} \frac{d t}{\log t}\right)-1\right\}+\left(\int_{2}^{\theta_{3}} \frac{d t}{\log t}-\pi_{3}\right)\right) \rightarrow \infty \\
\text { where } \quad\left(\int_{2}^{\theta_{3}} \frac{d t}{\log t}-\pi_{3}\right) \approx-2.3266013098834977
\end{array}
$$

By Theorem 5.21 the difference in values attained between the estimate of the tailored integral and the Gauss' logarithmic integral diverges:

$$
\lim _{n \rightarrow \infty}\left(L i_{(n)}-U B_{(n)}\right)=\lim _{n \rightarrow \infty}\left(\int_{\theta_{\mathcal{U}}}^{n} \frac{d t}{\log t}\right) \rightarrow \infty
$$

Consequently therefore:

$$
\lim _{n \rightarrow \infty}\left(L i_{(n)}-\pi_{(n)}\right) \rightarrow \infty
$$

Both $L i_{(n)}>\pi_{(n)}$ and $T L i_{(n)}>\pi_{(n)}$ for $n \in \mathbb{N} \mid n \geq 43$, from 6.16 we obtain:

$$
T L i_{(n)}-\pi_{(n)}<L i_{(n)}-\pi_{(n)} \quad \forall n \in \mathbb{N} \mid n \geq 43
$$

by Theorem 5.15 we have:

$$
\left(T L i_{(n)}-\pi_{(n)}\right)>\sum_{p \leq n} \frac{1}{p} \forall n \in \mathbb{N} \mid n \geq 983
$$


Consequently,

$$
\left(L i_{(n)}-\pi_{(n)}\right)>\left(T L i_{(n)}-\pi_{(n)}\right)>\sum_{p \leq n} \frac{1}{p} \quad \forall n \in \mathbb{N} \mid n \geq 983
$$

Direct evaluation confirms that:

$$
\left(L i_{(n)}-\pi_{(n)}\right)>\sum_{p \leq n} \frac{1}{p} \quad \forall n \in \mathbb{N} \mid 53 \leq n \leq 983
$$

Please refer to the Table 7 in the Appendix. Therefore, the sum of reciprocals of prime numbers is for all $n \in \mathbb{N} \mid n \geq 53$, the primary lower bound of the estimation error, made by the application of the Gauss' logarithmic integral. Concluding the proof of Theorem 6.6.

\subsection{Lower bound on the error term of the Gauss' logarithmic integral.}

By Theorem 6.6 we have that the sum of reciprocals of prime numbers for all $n \in \mathbb{N} \mid n \geq 53$ is the primary lower bound on the estimation error made by the application of the Gauss' logarithmic integral. However, despite the fact that this bound may well serve its purpose, it is far from being optimal and evidently does not prove equation 6.21 .

Theorem 6.7 (Lower Estimation Error Bound on The Logarithmic Integral).

For any $n \in \mathbb{N} \mid n \geq 4$, the error made in estimation of the true value of $\pi_{(n)}$ by the application of the logarithmic integral is bounded below by:

$$
\begin{array}{r}
\operatorname{Gauss}_{\mathcal{L E B}} \mathcal{B}_{(n)}=\frac{1}{2 \gamma}\left(\frac{\sqrt{n}}{\left(\log _{10} n\right)^{2}}+\frac{5}{\log 10}\left(\log _{10} n\right)-6\right)-\left(\frac{\left.(\exp (2))\left(\log _{10} n\right)^{(\sqrt{3}-1}\right)}{\mathcal{A}(\log 10)}\right) \\
\leq\left(L i_{(n)}-\pi_{(n)}\right)
\end{array}
$$

Proof.

The Theorem 6.4 states that:

$$
\left(\frac{\left(\log _{10} n\right)^{(\sqrt{3}-1)}}{\mathcal{A}(\log 10)}\right)\left(T L i_{(n)}-\pi_{(n)}\right) \leq\left(L i_{(n)}-\pi_{(n)}\right) \quad \forall n \in \mathbb{N} \mid n \geq 11
$$

Theorem 5.25 states that the lower estimation error bound on the tailored logarithmic integral is given by:

$$
\begin{aligned}
\mathcal{L E B}_{(n)}=\frac{\mathcal{A}(\log 10)}{2 \gamma\left(\log _{10} n\right)^{(\sqrt{3}-1)}}( & \left.\frac{\sqrt{n}}{\left(\log _{10} n\right)^{2}}+\frac{5}{\log 10}\left(\log _{10} n\right)-6\right)-(\exp (2)) \\
& \leq T L i_{(n)}-\pi_{(n)} \text { for any } n \in \mathbb{N} \text { s.t. } n \geq 11
\end{aligned}
$$


Therefore for all $n \in \mathbb{N} \mid n \geq 11$,

$$
\begin{aligned}
& \left(\frac{\left(\log _{10} n\right)^{(\sqrt{3}-1)}}{\mathcal{A}(\log 10)}\right) \times(\mathcal{L E \mathcal { B }}(n)) \\
& =\frac{1}{2 \gamma}\left(\frac{\sqrt{n}}{\left(\log _{10} n\right)^{2}}+\frac{5}{\log 10}\left(\log _{10} n\right)-6\right)-\left(\frac{(\exp (2))\left(\log _{10} n\right)^{(\sqrt{3}-1)}}{\mathcal{A}(\log 10)}\right) \\
& \leq\left(\frac{\left(\log _{10} n\right)^{(\sqrt{3}-1)}}{\mathcal{A}(\log 10)}\right)\left(T L i_{(n)}-\pi_{(n)}\right) \leq\left(L i_{(n)}-\pi_{(n)}\right)
\end{aligned}
$$

Direct computation verifies that the lower bound is strictly less than $\left(L i_{(n)}-\pi_{(n)}\right)$ for all $n \in \mathbb{N} \mid 4 \leq n \leq 11$ with the difference at $n=4$ :

$$
\left(L i_{(n)}-\pi_{(n)}\right)-\mathcal{L} \mathcal{E} \mathcal{B}_{(n)} \approx 0.933759
$$

Consequently, Theorem 6.7 holds for all $n \in \mathbb{N} \mid n \geq 4$, concluding the proof.

\subsection{Upper bound on the error term of the Gauss' logarithmic integral.}

Theorem 6.8 (Upper Estimation Error Bound on The Gauss' Logarithmic Integral).

For any $n \in \mathbb{N} \mid n \geq 3$, the error made in estimation of the true value of $\pi_{(n)}$ by the application of the logarithmic integral is bounded above by:

$$
\operatorname{Gauss} \mathcal{U E B}_{(n)}=2 \gamma\left(\frac{\sqrt{n}}{\left(\log _{10} n\right)}+\frac{5\left(\log _{10} n\right)}{(\log 10)}+7\right)+3.5 \geq\left(L i_{(n)}-\pi_{(n)}\right)
$$

Proof.

Theorem 6.5 states that for all $n \in \mathbb{N} \mid n \geq 3$ :

$$
\frac{2 \gamma}{\mathcal{T C}}\left(\log _{10} n\right)^{\sqrt{2}}\left(T L i_{(n)}-\pi_{(n)}\right)+3.5 \geq\left(L i_{(n)}-\pi_{(n)}\right)
$$

Theorem 5.26 states that the upper error bound on the tailored logarithmic integral estimation error is given by:

$$
\begin{array}{r}
\leq \mathcal{U E B} \mathcal{B}_{(n)}=\mathcal{T C}\left(\frac{\sqrt{n}}{\left(\log _{10} n\right)^{(\sqrt{2}+1)}}+\frac{5\left(\log _{10} n\right)^{2}}{(\log 10)\left(\log _{10} n\right)^{(\sqrt{2}+1)}}+\frac{7}{\left(\log _{10} n\right)^{\sqrt{2}}}\right) \\
\text { for all } n \in \mathbb{N} \mid n \geq 3
\end{array}
$$

Consequently for all $n \in \mathbb{N} \mid n \geq 3$,

$$
\begin{aligned}
\frac{2 \gamma}{\mathcal{T C}}\left(\log _{10} n\right)^{\sqrt{2}} & \times\left(\mathcal{U E \mathcal { B }}{ }_{(n)}\right)+3.5=2 \gamma\left(\frac{\sqrt{n}}{\left(\log _{10} n\right)}+\frac{5\left(\log _{10} n\right)}{(\log 10)}+7\right)+3.5 \\
& \geq \frac{2 \gamma}{\mathcal{T C}}\left(\log _{10} n\right)^{\sqrt{2}}\left(T L i_{(n)}-\pi_{(n)}\right)+3.5 \geq\left(L i_{(n)}-\pi_{(n)}\right)
\end{aligned}
$$

Direct computation verifies that the upper bound is strictly greater than $\left(L i_{(n)}-\pi_{(n)}\right)$ at $n=2$ :

$$
\mathcal{U E \mathcal { B } _ { ( n ) }}-\left(L i_{(n)}-\pi_{(n)}\right) \approx 18.7591
$$

Therefore, Theorem 6.8 holds for all $n \in \mathbb{N} \mid n \geq 2$, concluding the proof. 
6.6. Estimation error bounds $\left(L i_{(n)}-\pi_{(n)}\right)$ and $\left(T L i_{(n)}-\pi_{(n)}\right)$ summary.

The $\left(L i_{(n)}-\pi_{(n)}\right)$ estimation error bounds are given by Theorems 6.7 and 6.8 :

$$
\begin{aligned}
& \frac{1}{2 \gamma}\left(\frac{\sqrt{n}}{\left(\log _{10} n\right)^{2}}+\frac{5}{\log 10}\left(\log _{10} n\right)-6\right)-\left(\frac{(\exp (2))\left(\log _{10} n\right)^{(\sqrt{3}-1)}}{\mathcal{A}(\log 10)}\right) \\
& \quad \leq\left(L i_{(n)}-\pi_{(n)}\right) \leq 2 \gamma\left(\frac{\sqrt{n}}{\left(\log _{10} n\right)}+\frac{5\left(\log _{10} n\right)}{(\log 10)}+7\right)+3.5 \forall n \in \mathbb{N} \mid n \geq 4
\end{aligned}
$$

In an analogous way by Theorems 5.26 and 5.25 we have:

$$
\begin{array}{r}
\frac{\mathcal{A}(\log 10)}{2 \gamma\left(\log _{10} n\right)^{(\sqrt{3}-1)}}\left(\frac{\sqrt{n}}{\left(\log _{10} n\right)^{2}}+\frac{5}{\log 10}\left(\log _{10} n\right)-6\right)-(\exp (2)) \leq\left(T L i_{(n)}-\pi_{(n)}\right) \\
\leq \frac{\mathcal{T C}}{\left(\log _{10} n\right)^{\sqrt{2}}}\left(\frac{\sqrt{n}}{\log _{10} n}+\frac{5\left(\log _{10} n\right)^{2}}{\log n}+7\right) \\
\quad \forall n \in \mathbb{N} \mid n \geq 11
\end{array}
$$

The Infimum and Supremum estimation error bounds are given by Theorems 5.16 and 5.17:

$$
\begin{aligned}
& \sum_{k=1}^{\left(\pi_{(n)}-14\right)}\left\{\frac{\log p_{(14+k)}}{\log \left(\log p_{(14+k)} \sharp\right)}+\frac{1}{5\left(p_{(14+k)}\right)}-1\right\} \\
& <T L i_{(n)}-\pi_{(n)}<\sum_{k=1}^{\left(\pi_{(n)}-14\right)}\left\{\frac{\log p_{(14+k)}}{\log \left(\log p_{(14+k)} \sharp\right)}+\frac{1}{p_{(14+k)}}-1\right\} \\
& \forall n \in \mathbb{N} \mid n \geq 47 \text { where } p_{(n)} \text { is the greatest prime number } p \in \mathbb{N} \mid p \leq n
\end{aligned}
$$

\subsection{Riemann's hypothesis.}

Riemann's hypothesis is the final major objective of this research.

Theorem 6.9 (The Riemann's Hypothesis).

The prime counting function $\pi_{(n)}$ is given by:

$$
\pi_{(n)}=L i_{(n)}+\mathcal{O}(\sqrt{n} \log n) \quad \forall n \in \mathbb{N} \mid n \geq 2
$$

Proof.

By Theorem 6.8 we have that:

$$
\pi_{(n)}=\operatorname{Li}_{(n)}+\mathcal{O}\left(\operatorname{Gauss} \mathcal{U E}_{(n)}\right) \quad \forall n \in \mathbb{N} \mid n \geq 2
$$

Where:

$$
\operatorname{Gauss\mathcal {UEB}}_{(n)}=2 \gamma\left(\frac{\sqrt{n}}{\left(\log _{10} n\right)}+\frac{5\left(\log _{10} n\right)}{(\log 10)}+7\right)+3.5
$$

Suppose that:

$$
\operatorname{GaussUE}_{(n)}>\sqrt{n} \log n
$$

Necessarily therefore,

$$
\frac{\sqrt{n} \log n}{\operatorname{GaussU\mathcal {E}}_{(n)}}<1
$$


By L'Hôpital's rule we obtain:

$$
\begin{gathered}
\lim _{n \rightarrow \infty}\left(\frac{\sqrt{n} \log n}{2 \gamma\left(\frac{\sqrt{n}}{\log _{10} n}+\frac{5\left(\log _{10} n\right)}{\log 10}+7\right)+3.5}\right)= \\
\quad=\lim _{n \rightarrow \infty}\left(\frac{(2+\log n)}{2 \gamma\left(\left(\frac{1}{\log n}-\frac{2}{(\log n)^{2}}\right) \log 10+\frac{10}{\sqrt{n}(\log 10)^{2}}\right)}\right)
\end{gathered}
$$

Every term in the denominator of 6.32 tends to zero as $n$ increases unboundedly, while the numerator diverges. Necessarily therefore,

$$
\lim _{n \rightarrow \infty}\left(\frac{\sqrt{n} \log n}{2 \gamma\left(\frac{\sqrt{n}}{\log _{10} n}+\frac{5\left(\log _{10} n\right)}{\log 10}+7\right)+3.5}\right) \rightarrow \infty
$$

Direct computation verifies that the ratio 6.31 attains the value $\approx 0.0551976$ at $n=2$ and further increases. At $n=33$ it exceeds the value of 1 and decisively diverges. For all $n \in \mathbb{N} \mid n \geq 967$ the ratio 6.31 diverges at a rate exceeding $\log (n)$, while further accelerating. Thus we have a contradiction to the initial hypothe-

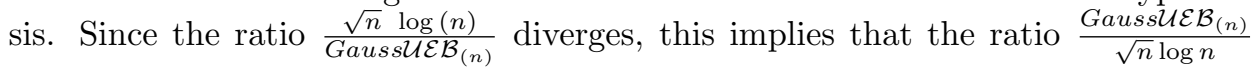
asymptotically converges to zero, as $n$ increases unboundedly. Analogous numerical computation confirms that at $n=2$ the difference:

$$
\sqrt{n} \log n-\left(L i_{(n)}-\pi_{(n)}\right) \approx 1.98026
$$

and diverges. Therefore, for all $n \in \mathbb{N} \mid n \geq 2$ :

$$
\operatorname{Gauss\mathcal {E}}_{(n)}=o(\sqrt{n} \log n)
$$

Since

$$
\pi_{(n)}=L i_{(n)}+\mathcal{O}\left(\text { Gauss } \mathcal{U E B}_{(n)}\right)
$$

this of course implies that

$$
\pi_{(n)}=L i_{(n)}+\mathcal{O}(\sqrt{n} \log n)
$$

Concluding the proof of the Riemann's hypothesis. 


\section{REFERENCES}

[1] R.J. Backlund, Über die Differenzen zwischen den Zahlen, die zu den ersten $n$ Primzahlen teilerfremd sind. Commentationes in honorem Ernesti Leonardi Lindelöf, Annales Acad. Sci. Fenn. 32 (1929), no. 2, 1-9.

[2] C. Bays and R.H. Hudson, A new bound for the smallest $x$ with $\pi_{x}>L i_{x}$, Mathematics Of Computation (1999).

[3] Enrico Bombieri, The Riemann Hypothesis, Clay Mathematics Institute (2000).

[4] _ The shifting aspects of truth in mathematics, Institute For Advanced Study Princeton (2008).

[5] A. Brauer and H. Zeitz, Über eine zahlentheoretische Behauptung von Legendre, Sitzungsbericht, Berliner Math. Ges. 29 (1930), 116-125.

[6] Viggo Brun, Über das Goldbachsche Gesetz und die Anzahl der Primzahlpaare, Arch. Mat. Natur (1915).

[7] Chris K. Caldwell, The gaps between primes, 2006. http://primes.utm.edu/notes/gaps.html.

[8] F. C. Kuok and R. Plymen, A new bound for the smallest $x$ with $\pi_{x}>L i_{x}, 2008$. http://arXiv:math/0509312v6[math.NT].

[9] Calvin C. Clawson, Mathematical mysteries; the beauty and magic of numbers, Plenum Press, New York, 1996.

[10] Harvey Cohn, Advanced number theory, Dover Publications, New York, 1980.

[11] J. Brian Conrey, The Riemann Hypothesis, Notices Of The AMS (2003).

[12] Harald Cramer, On the order of magnitude of the difference between consecutive prime numbers, Acta Arithmetica (1936).

[13] R. Crandall and C. Pomerance, Prime numbers, a computational perspective, Springer Verlag, New York, 2005.

[14] Paul Erdös, On the difference of consecutive primes, Quarterly Journal Of Mathematics (1935).

[15] Some unsolved problems, Publications Of The Mathematical Institute Of The Hungarian Academy Of Sciences (1961).

[16] A survey of problems in combinatorial number theory, Annals Of Discrete Mathematics (1980)

[17] Jan Feliksiak, The elementary proof of the Riemann's Hypothesis, MDPI, AG, https://doi.org/10.20944/preprints202006.0365.v1 (2020).

[18] _ The maximal prime gaps Supremum and the Firoozbakht's Hypothesis No 30, MDPI, AG, https://doi.org/10.20944/preprints202006.0366.v1 (2020).

[19] _ Maximal prime gaps bounds, ScienceOpen, Inc, https://doi.org/10.14293/S21991006.1.SOR-.PPWVKRR.v1 (2021).

[20] The Binary Goldbach Conjecture, ScienceOpen, Inc, https://doi.org/10.14293/S2199-1006.1.SOR-.PPIU46T.v1 (2021).

[21] Andrew Granville, Harald Cramer and the distribution of prime numbers, Scandinavian Actuarial Journal (1995).

[22] G.H. Hardy and E.M. Wright, An introduction to the theory of numbers, Oxford University Press, London, 1968.

[23] Ki H. Kim and Fred W. Roush, Applied abstract algebra, John Wiley and Sons, New York, 1999.

[24] Helge von Koch, Sur la distribution des nombres premiers, Acta Mathematica (1901).

[25] Edmund Landau, Vorlesungen über Zahlentheorie, Vol. II, S. Hirzel, Leipzig, 1927.

[26] H Maier, Primes in short intervals, Michigan Mathematical Journal (1985).

[27] Thomas R. Nicely, Some results of computational research in prime numbers (Computational number theory), 2009. http://www.trnicely.net.

[28] _ Skewes' problem, 2009. http://www.trnicely.net.

[29] János Pintz, Very large gaps between consecutive primes, Journal Of Number Theory (1997).

[30] S. Ramanujan, A proof of Bertrand's postulate, Journal Of Indian Mathematical Society 11 (1919), 181-182.

[31] Robert A. Rankin, The difference between consecutive prime numbers, Journal London Mathematical Society (1938).

[32] P. Ribenboim, The new book of prime number records, Springer Verlag, New York, 1996. 
[33] Hermann J. J. te Riele, On the sign of the difference $\pi_{x}-L i_{x}$, Mathematics Of Computation (1987).

[34] Sebastian M. Ruiz, A result on prime numbers, Mathematical Gazette 81 (1997), no. 269, 269.

[35] Marcus du Sautoy, The music of primes, Harper Collins, New York, 2003.

[36] Atle Selberg, On the normal density of primes in small intervals and the difference between consecutive primes, Arch. Mathem. Naturv. B 47 (1943), 87-105.

[37] Daniel Shanks, On maximal gaps between successive primes, Math. Comp. 18 (1964), 646651.

[38] Tomas Oliveira e Silva, Gaps between consecutive primes, 2006. www.ieeta.pt/ tos/gaps.html.

[39] K. Soundararajan, Small gaps between prime numbers: the work of Goldston-Pintz-Yildirim, Bulletin of the American Mathematical Society (2007).

[40] Jeffrey Stopple, A primer of analytic number theory, Cambrige University Press, Cambrige, 2003.

[41] Eric W. Weisstein, Khinchin's constant, 1996. www.mathworld.wolfram.com/KhinchinsConstant.html.

[42] E. Westzynthius, Über die Differenzen Verteilung der Zahlen die zu den $n$ ersten Primzahlen teilerfremd sind, Comm. Phys. Math. Soc. Sci. Fenn. 5 (1931), 1-37.

[43] Song Y. Yan, Number theory for computing, Springer Verlag, Heidelberg, 2002. 


\section{ApPENDiX}

\subsection{Graphical comparison of functions.}

The drawings 13 to 17 show the graphs of the upper and lower estimation error bounds (red) and the estimation error $T L i_{\left(p_{n}\right)}-\pi_{\left(p_{n}\right)}$ (blue). Due to the fact that the estimation error curve $T L i_{\left(p_{n}\right)}-\pi_{\left(p_{n}\right)}$ is relatively smooth, to reduce the size of the resultant database, a technique had been implemented in construction of the figures: 13 to 16 . The figures were produced by computing the distance from the curve $T L i_{\left(p_{n}\right)}-\pi_{\left(p_{n}\right)}$ to each bound, at every prime within a sub-interval. Two individual points exhibiting the least distance were stored per interval. The interval widths for all $n \in \mathbb{N} \mid n \leq 10^{9}$ were computed in accordance with the criteria:

TABLE 5. Interval widths specifications

\begin{tabular}{|c|c|}
\hline $\mathrm{n}$ & Interval width \\
\hline$<10^{3}$ & 50 \\
\hline$<10^{5}$ & 200 \\
\hline$<10^{6}$ & $10^{3}$ \\
\hline$<10^{7}$ & $10^{4}$ \\
\hline$<10^{8}$ & $5 * 10^{4}$ \\
\hline$<10^{9}$ & $10^{5}$ \\
\hline$<10^{10}$ & $10^{6}$ \\
\hline above & $10^{7}$ \\
\hline
\end{tabular}

The graphs had been constructed in such a way that their ranges slightly overlap. The points exhibiting locally/globally least distance from either estimation error bound are indicated on the graphs by a black dot located on the respective bound curve. Table 6 , specifies the coordinates of such points.

TABLE 6. Critical points - minima

\begin{tabular}{|l|l|l|c|}
\hline $\mathrm{n}$ & $\xi$ & $\mathcal{U E B}_{(n)}-\left(T L i_{n}-\pi_{n}\right)$ & attribute \\
\hline 1866373 & 3.5435062790146286 & 0.4783128416250477 & local \\
\hline 3189553 & 3.71212639526346 & 0.4276359962528389 & local \\
\hline 4890913 & 3.8466421193071674 & 0.3706127200088041 & local \\
\hline 6862489 & 3.953214011532586 & 0.3374931519939466 & global \\
\hline 10675969 & 4.092269278529259 & 0.42793313427111457 & local \\
\hline 16957337 & 4.237863185491645 & 0.4720328466920449 & local \\
\hline \hline $\mathrm{n}$ & $\xi$ & $\left(T L i_{n}-\pi_{n}\right)-\mathcal{L E \mathcal { B }}(n)$ & attribute \\
\hline 34189626 & 4.45850818707939 & 0.4403051640949798 & local \\
\hline 84620646 & 4.743668868936391 & 0.39131309508213974 & local \\
\hline 118888260 & 4.850654400251596 & 0.3233165712131978 & local \\
\hline 197430720 & 5.010250084067886 & 0.14750273586641072 & global \\
\hline 319028868 & 5.161252515015835 & 0.5455210759252225 & local \\
\hline 502777012 & 5.304379467814857 & 0.2490621490423166 & local \\
\hline 729743022 & 5.421603942627343 & 1.1090294068878563 & local \\
\hline 876080592 & 5.479112609478988 & 1.8641473315881 & local \\
\hline
\end{tabular}




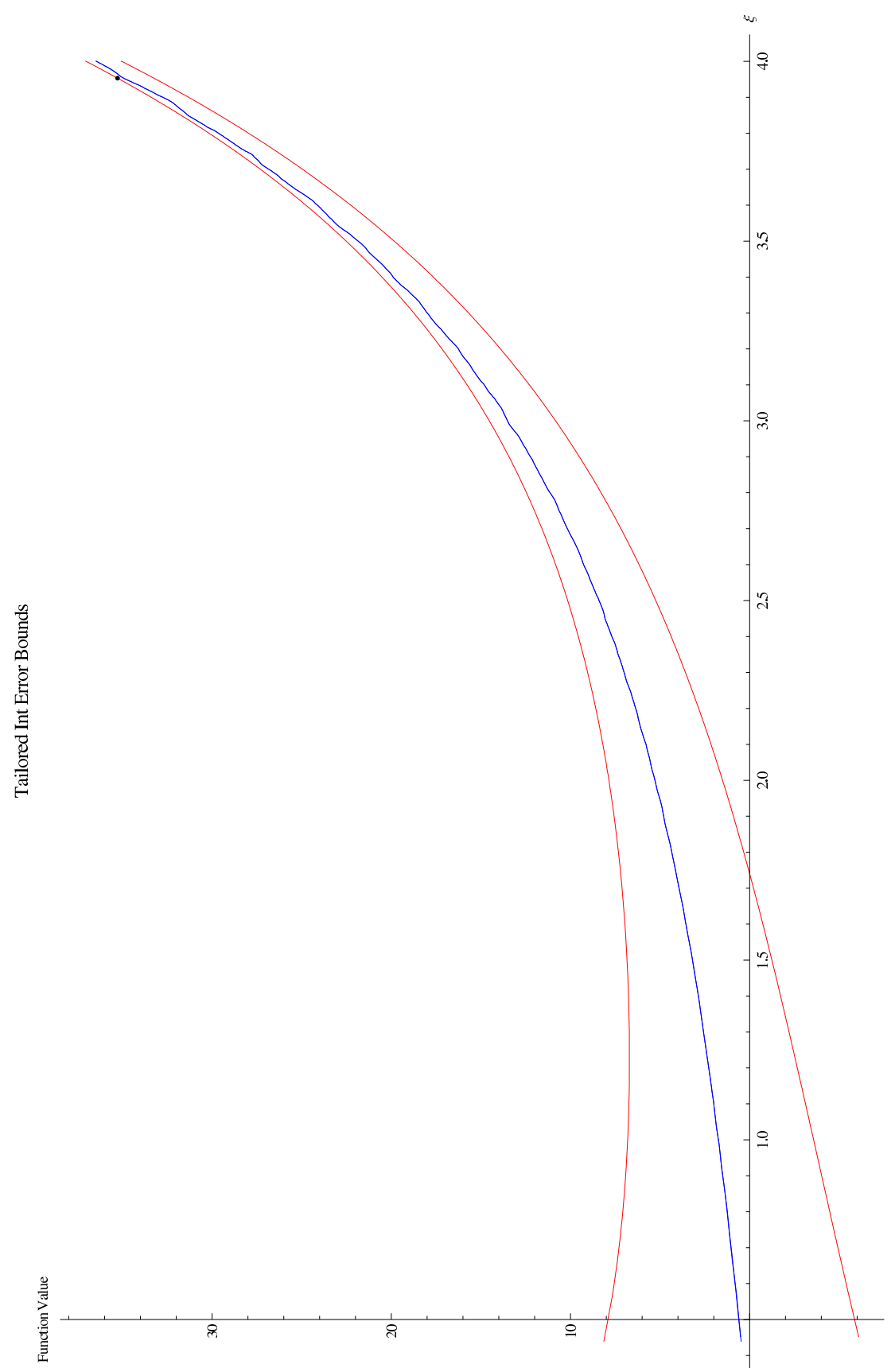

FIGURE 13. The figure is drawn with respect to $\xi$, which gives the range $n \in \mathbb{N} \mid 11 \leq n \leq 7975013$, which includes the global minimum point at $n=6862489, \xi \approx 3.9532140115325856$. Please refer to Table 6. 


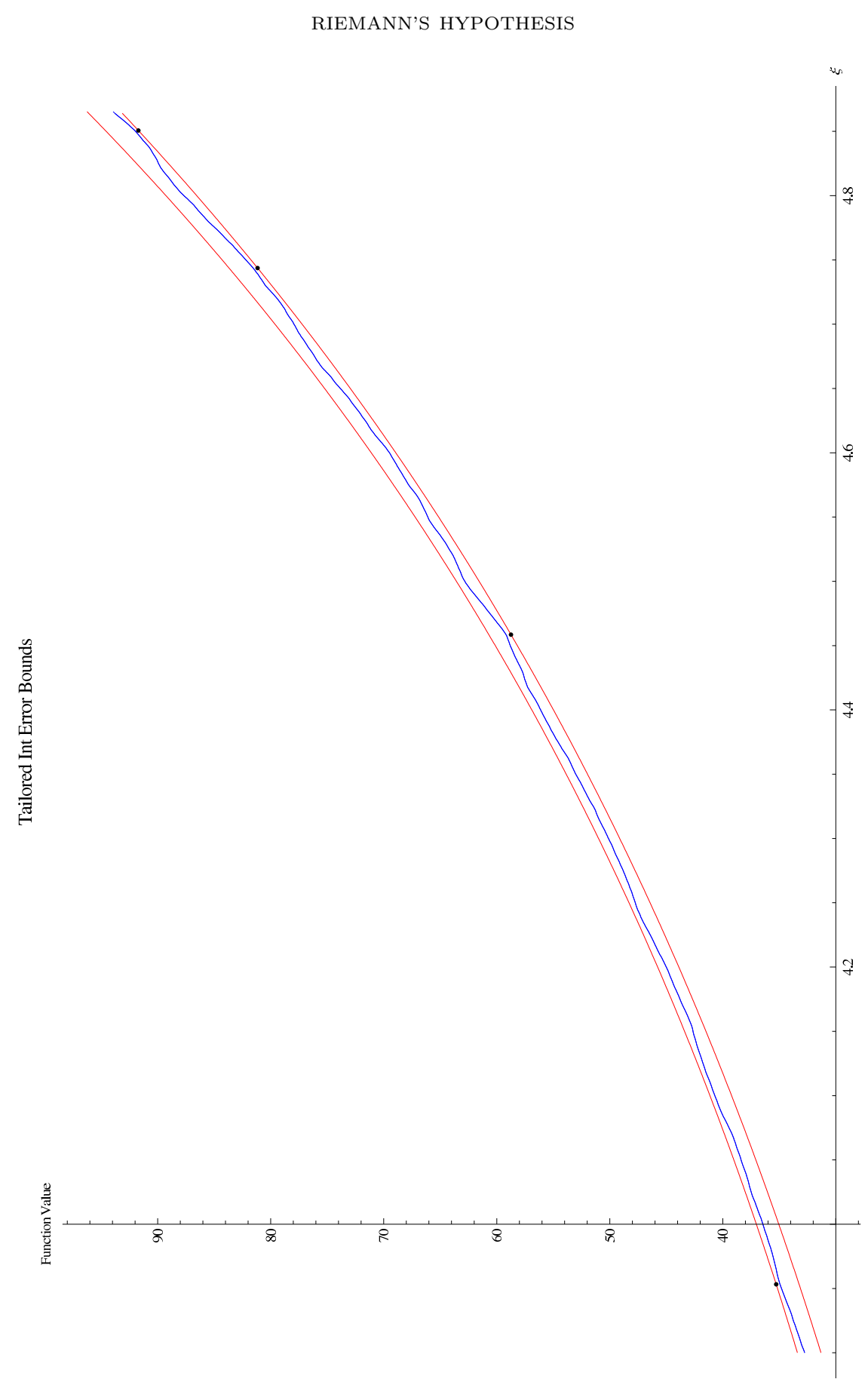

FiguRE 14. The figure is drawn with respect to $\xi$, which gives the range $n \in \mathbb{N} \mid 5799991 \leq n \leq 124499941$. 


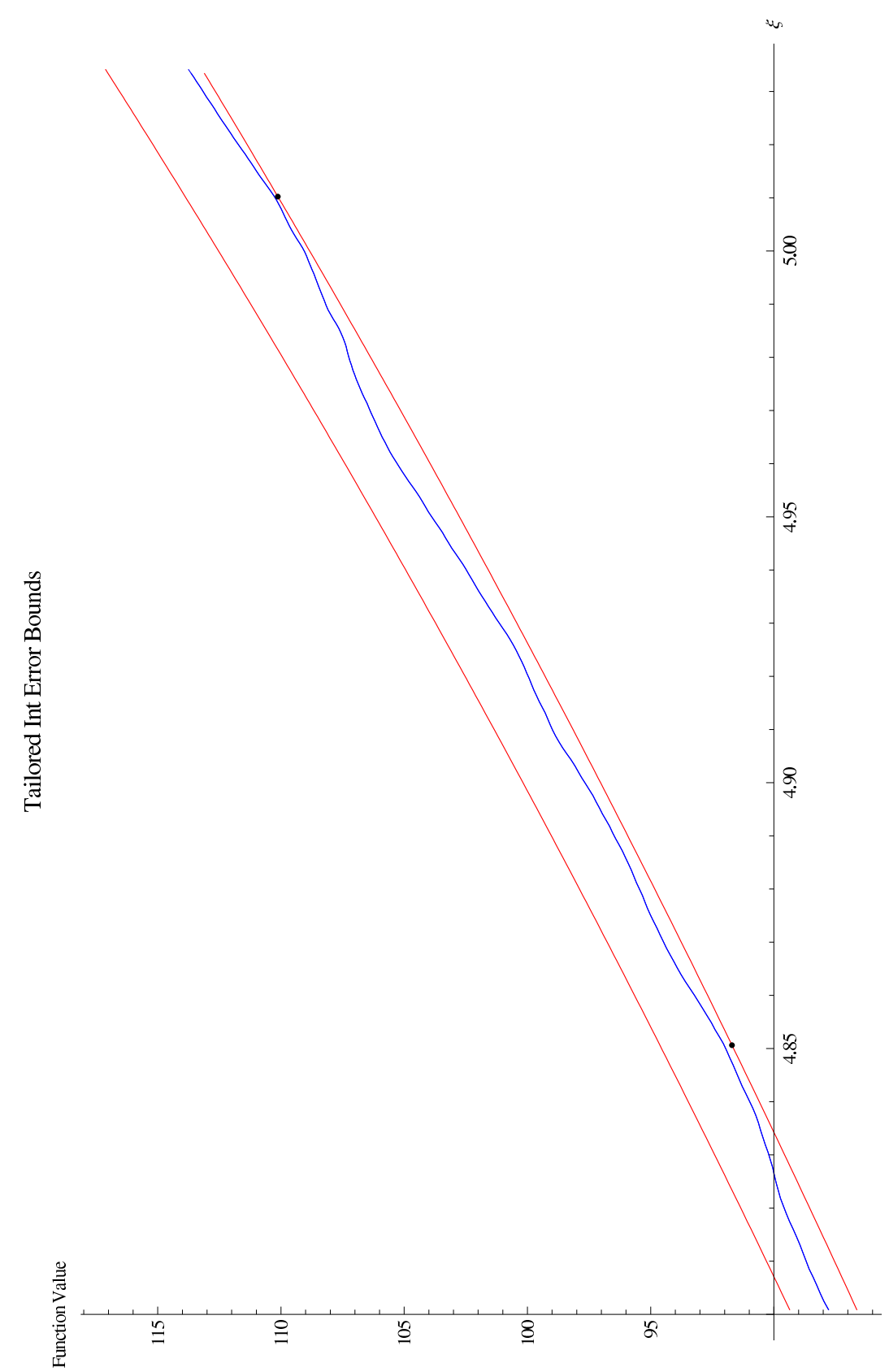

Figure 15. The figure is drawn with respect to $\xi$, which gives the range $n \in \mathbb{N} \mid 101499997 \leq n \leq 212999959$. The graph includes the global minimum at $n=197430720, \xi \approx 5.010250084067886$. Please refer to Table 6 . 


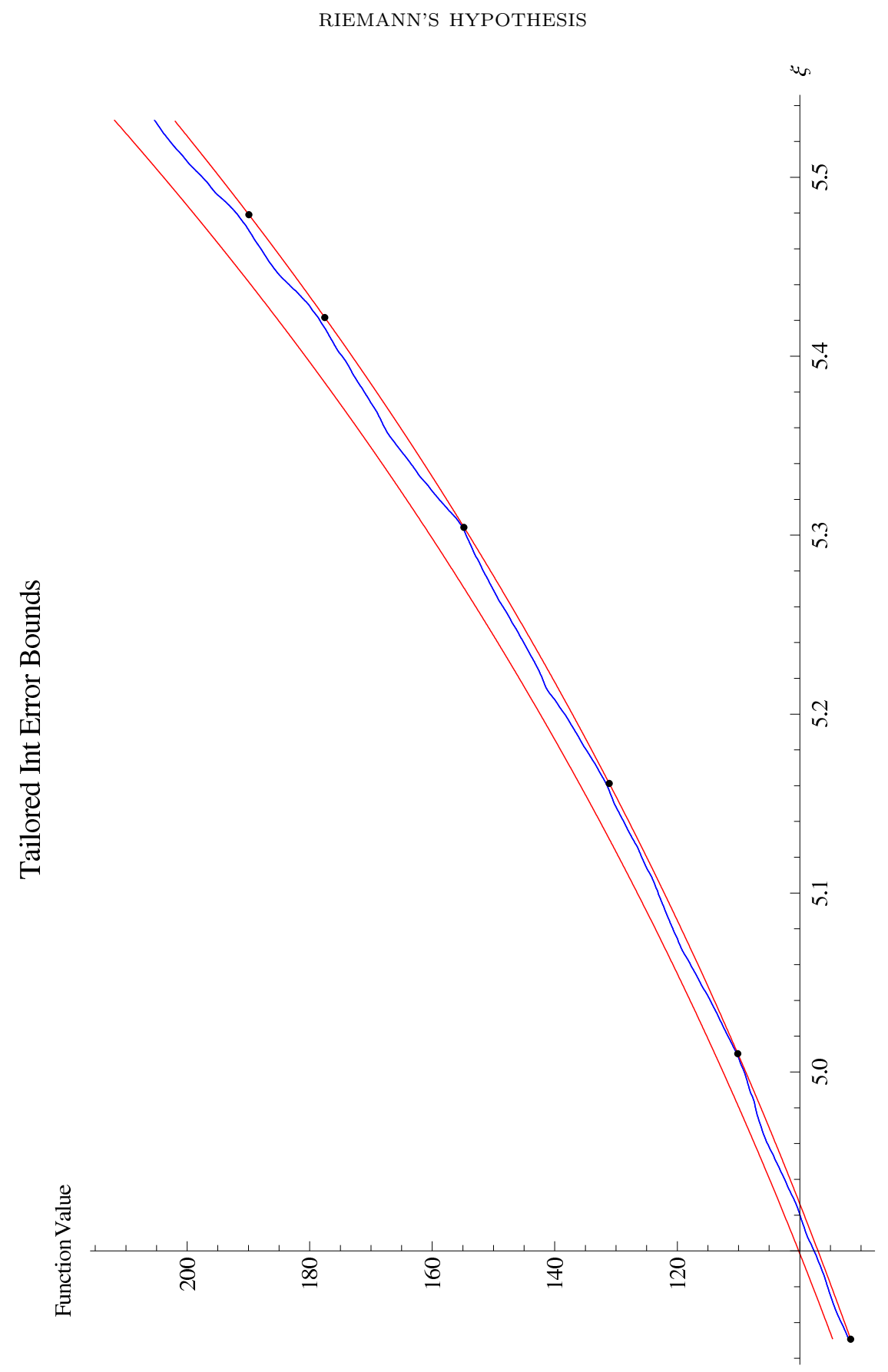

Figure 16. The figure is drawn with respect to $\xi$, which gives the range $n \in \mathbb{N} \mid 118888261 \leq n \leq 1036120507$. The graph includes the global minimum at $n=197430720, \xi \approx 5.010250084067886$, as well as other minima given in Table 6 . 


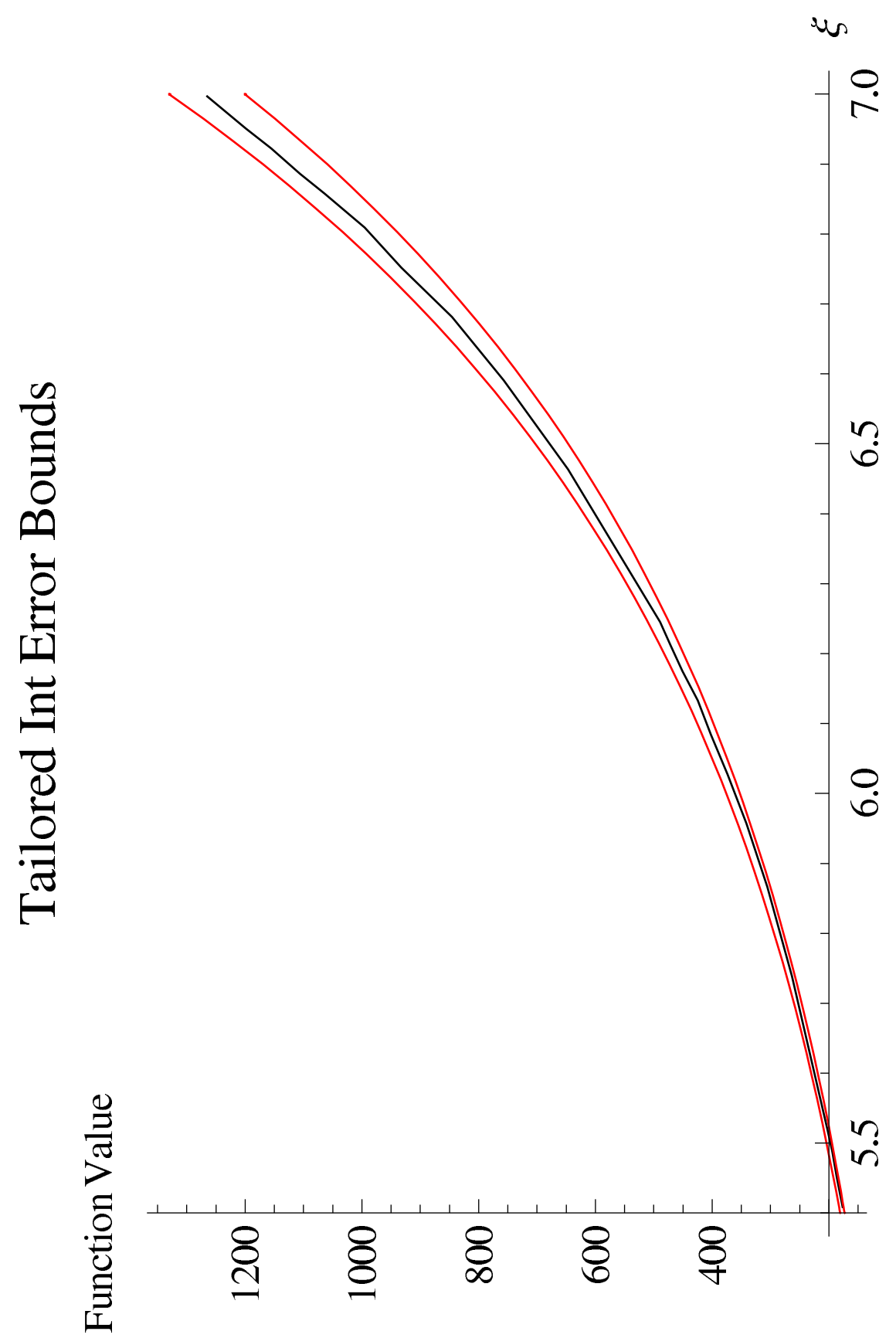

Figure 17. The figure is drawn with respect to $\xi$, which gives the range $n \in \mathbb{N} \mid 700000001 \leq n \leq 108965188117$. 


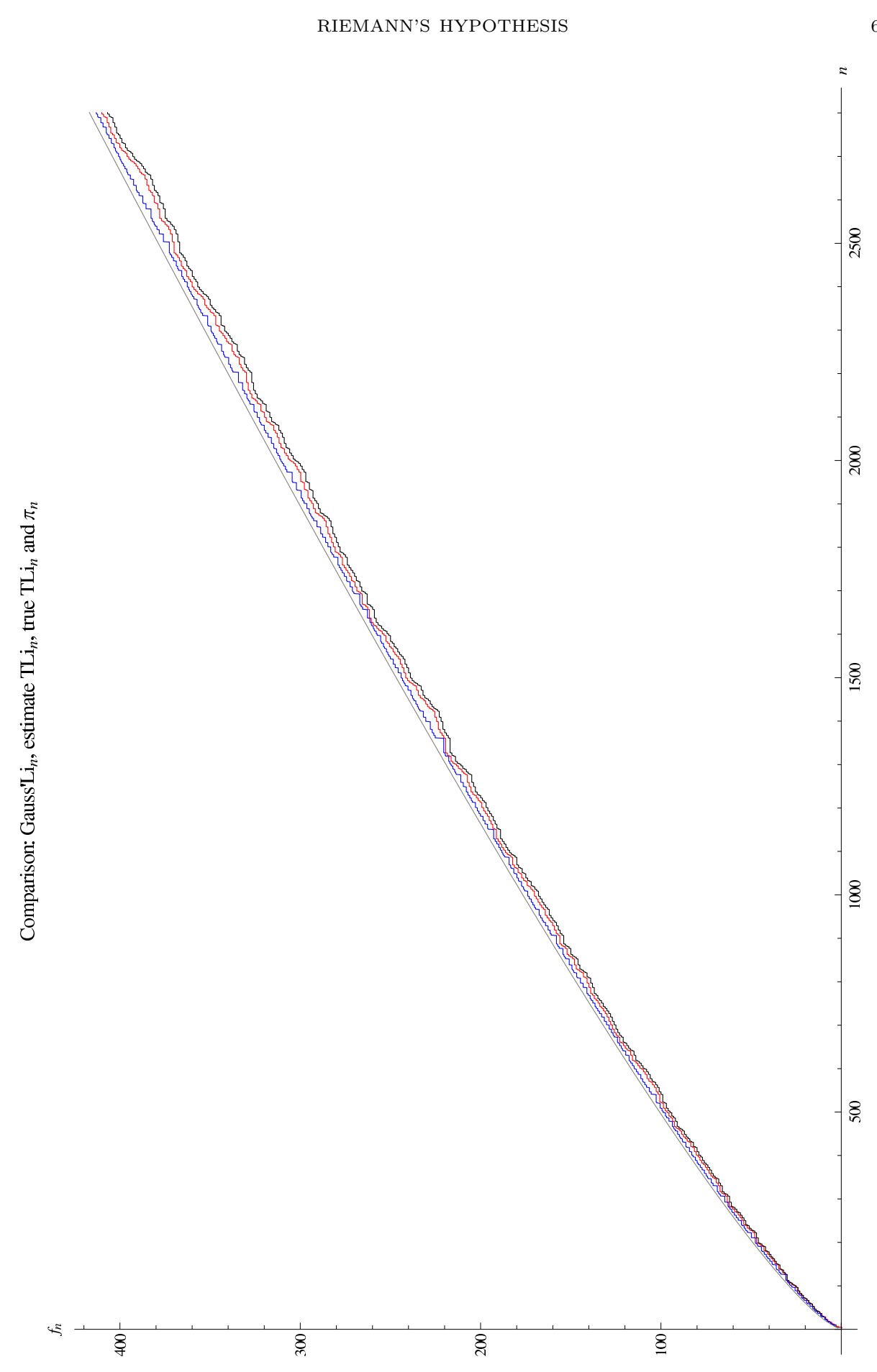

Figure 18. Characteristic behaviour of $U B_{(n)}$ (blue) in places where $\pi_{(n)}$ (black) and $\operatorname{Sup}_{(n)}$ (red) tend to "sag" 


\subsection{Tabular data.}

TABLE $7 . L i_{n}-\pi_{n}$ less the $\sum_{p \leq n} 1 / p$

\begin{tabular}{|c|c|c|c|}
\hline \multicolumn{4}{|c|}{ Primary Lower Bound } \\
\hline $\mathrm{n}$ & $L i_{n}-\pi_{n}-\sum 1 / p$ & $\mathrm{n}$ & $L i_{n}-\pi_{n}-\sum 1 / p$ \\
\hline 47 & -0.0110464 & 467 & 2.32106 \\
\hline 53 & 0.504175 & 479 & 3.26732 \\
\hline 59 & 0.978044 & 487 & 3.55977 \\
\hline 61 & 0.450138 & 491 & 3.20369 \\
\hline 67 & 0.878096 & 499 & 3.49106 \\
\hline 71 & 0.808767 & 503 & 3.13251 \\
\hline 73 & 0.262727 & 509 & 3.09417 \\
\hline 79 & 0.635637 & 521 & 4.01405 \\
\hline 83 & 0.533859 & 523 & 3.33175 \\
\hline 89 & 0.86971 & 541 & 5.1977 \\
\hline 97 & 1.62457 & 547 & 5.14841 \\
\hline 101 & 1.48517 & 557 & 5.73052 \\
\hline 103 & 0.907901 & 563 & 5.67692 \\
\hline 107 & 0.758054 & 569 & 5.62174 \\
\hline 109 & 0.176037 & 571 & 4.93517 \\
\hline 113 & 0.0165432 & 577 & 4.87793 \\
\hline 127 & 1.93345 & 587 & 5.44696 \\
\hline 131 & 1.7489 & 593 & 5.38569 \\
\hline 137 & 1.96666 & 599 & 5.32296 \\
\hline 139 & 1.36537 & 601 & 4.63394 \\
\hline 149 & 2.37092 & 607 & 4.56927 \\
\hline 151 & 1.76345 & 613 & 4.50318 \\
\hline 157 & 1.9483 & 617 & 4.12445 \\
\hline 163 & 2.12441 & 619 & 3.43405 \\
\hline 167 & 1.90183 & 631 & 4.29647 \\
\hline 173 & 2.06433 & 641 & 4.84405 \\
\hline 179 & 2.2192 & 643 & 4.15188 \\
\hline 181 & 1.59881 & 647 & 3.76864 \\
\hline 191 & 2.50724 & 653 & 3.69347 \\
\hline 193 & 1.88247 & 659 & 3.617 \\
\hline 197 & 1.63597 & 661 & 2.92355 \\
\hline 199 & 1.00914 & 673 & 3.76743 \\
\hline 211 & 2.25885 & 677 & 3.37995 \\
\hline 223 & 3.48497 & 683 & 3.29843 \\
\hline 227 & 3.2191 & 691 & 3.52166 \\
\hline 229 & 2.5831 & 701 & 4.04804 \\
\hline 233 & 2.31378 & 709 & 4.26648 \\
\hline 239 & 2.40774 & 719 & 4.78696 \\
\hline 241 & 1.76851 & 727 & 5.00076 \\
\hline 251 & 2.58098 & 733 & 4.90944 \\
\hline \multicolumn{4}{|c|}{ Continued } \\
\hline
\end{tabular}


TABLE 7. Continued

\begin{tabular}{|l|l|l|l|}
\hline \multicolumn{3}{|c|}{ Primary Lower Bound (Continued) } \\
\hline 257 & 2.66064 & 739 & 4.81701 \\
\hline 263 & 2.73585 & 743 & 4.42099 \\
\hline 269 & 2.80674 & 751 & 4.62884 \\
\hline 271 & 2.16029 & 757 & 4.53313 \\
\hline 277 & 2.22561 & 761 & 4.13495 \\
\hline 281 & 1.93238 & 769 & 4.33849 \\
\hline 283 & 1.28333 & 773 & 3.93891 \\
\hline 293 & 2.0458 & 787 & 5.03997 \\
\hline 307 & 3.49711 & 797 & 5.53695 \\
\hline 311 & 3.19157 & 809 & 6.32988 \\
\hline 313 & 2.53662 & 811 & 5.62728 \\
\hline 317 & 2.22881 & 821 & 6.11762 \\
\hline 331 & 3.64767 & 823 & 5.41439 \\
\hline 337 & 3.6772 & 827 & 5.00883 \\
\hline 347 & 4.38819 & 829 & 4.30529 \\
\hline 349 & 3.72707 & 839 & 4.79081 \\
\hline 353 & 3.40674 & 853 & 5.86664 \\
\hline 359 & 3.42525 & 857 & 5.45796 \\
\hline 367 & 3.77975 & 859 & 4.75289 \\
\hline 373 & 3.7917 & 863 & 4.34362 \\
\hline 379 & 3.80094 & 877 & 5.41089 \\
\hline 383 & 3.47141 & 881 & 4.99983 \\
\hline 389 & 3.47626 & 883 & 4.29359 \\
\hline 397 & 3.81292 & 887 & 3.88195 \\
\hline 401 & 3.47833 & 907 & 5.82244 \\
\hline 409 & 3.80836 & 911 & 5.40851 \\
\hline 419 & 4.46549 & 919 & 5.58063 \\
\hline 421 & 3.79423 & 929 & 6.04396 \\
\hline 431 & 4.4436 & 937 & 6.21276 \\
\hline 433 & 3.77086 & 941 & 5.79608 \\
\hline 439 & 3.75581 & 947 & 5.67092 \\
\hline 443 & 3.41047 & 953 & 5.54496 \\
\hline 449 & 3.3918 & 6.58268 \\
\hline 457 & 3.69768 & 6.16336 \\
\hline 461 & 3.34814 & & \\
\hline 463 & 2.67195 & End & \\
\hline & & & \\
\hline
\end{tabular}


TABLE 8. Theorem 3.6

\begin{tabular}{|c|c|c|c|}
\hline \multicolumn{4}{|c|}{ Maximal Prime Gaps Upper Bound } \\
\hline$N^{o}$ & Gap start $p_{n}$ & Actual gap $\mathfrak{g}$ & $\mathcal{U} \mathcal{B}_{\left(p_{n}\right)}$ \\
\hline 1 & 7 & 3 & 3 \\
\hline 2 & 23 & 5 & 9 \\
\hline 3 & 89 & 7 & 19 \\
\hline 4 & 113 & 13 & 21 \\
\hline 5 & 523 & 17 & 36 \\
\hline 6 & 887 & 19 & 43 \\
\hline 7 & 1129 & 21 & 46 \\
\hline 8 & 1327 & 33 & 48 \\
\hline 9 & 9551 & 35 & 79 \\
\hline 10 & 15683 & 43 & 88 \\
\hline 11 & 19609 & 51 & 92 \\
\hline 12 & 31397 & 71 & 101 \\
\hline 13 & 155921 & 85 & 134 \\
\hline 14 & 360653 & 95 & 154 \\
\hline 15 & 370261 & 111 & 155 \\
\hline 16 & 492113 & 113 & 161 \\
\hline 17 & 1349533 & 117 & 187 \\
\hline 18 & 1357201 & 131 & 188 \\
\hline 19 & 2010733 & 147 & 198 \\
\hline 20 & 4652353 & 153 & 222 \\
\hline 21 & 17051707 & 179 & 261 \\
\hline 22 & 20831323 & 209 & 267 \\
\hline 23 & 47326693 & 219 & 294 \\
\hline 24 & 122164747 & 221 & 326 \\
\hline 25 & 189695659 & 233 & 342 \\
\hline 26 & 191912783 & 247 & 343 \\
\hline 27 & 387096133 & 249 & 368 \\
\hline 28 & 436273009 & 281 & 373 \\
\hline 29 & 1294268491 & 287 & 415 \\
\hline 30 & 1453168141 & 291 & 419 \\
\hline 31 & 2300942549 & 319 & 438 \\
\hline 32 & 3842610773 & 335 & 459 \\
\hline 33 & 4302407359 & 353 & 464 \\
\hline 34 & 10726904659 & 381 & 503 \\
\hline 35 & 20678048297 & 383 & 532 \\
\hline 36 & 22367084959 & 393 & 535 \\
\hline 37 & 25056082087 & 455 & 540 \\
\hline 38 & 42652618343 & 463 & 564 \\
\hline 39 & 127976334671 & 467 & 616 \\
\hline 40 & 182226896239 & 473 & 634 \\
\hline 41 & 241160624143 & 485 & 647 \\
\hline 42 & 297501075799 & 489 & 658 \\
\hline
\end{tabular}


Table 8. Continued

\begin{tabular}{|c|c|c|c|}
\hline \multicolumn{4}{|c|}{ Maximal Prime Gaps Upper Bound (Continued) } \\
\hline 43 & 303371455241 & 499 & 659 \\
\hline 44 & 304599508537 & 513 & 659 \\
\hline 45 & 416608695821 & 515 & 675 \\
\hline 46 & 461690510011 & 531 & 680 \\
\hline 47 & 614487453523 & 533 & 694 \\
\hline 48 & 738832927927 & 539 & 704 \\
\hline 49 & 1346294310749 & 581 & 735 \\
\hline 50 & 1408695493609 & 587 & 737 \\
\hline 51 & 1968188556461 & 601 & 755 \\
\hline 52 & 2614941710599 & 651 & 770 \\
\hline 53 & 7177162611713 & 673 & 826 \\
\hline 54 & 13829048559701 & 715 & 863 \\
\hline 55 & 19581334192423 & 765 & 883 \\
\hline 56 & 42842283925351 & 777 & 929 \\
\hline 57 & 90874329411493 & 803 & 974 \\
\hline 58 & 171231342420521 & 805 & 1012 \\
\hline 59 & 218209405436543 & 905 & 1028 \\
\hline 60 & 1189459969825483 & 915 & 1136 \\
\hline 61 & 1686994940955803 & 923 & 1159 \\
\hline 62 & 1693182318746371 & 1131 & 1159 \\
\hline 63 & 43841547845541059 & 1183 & 1384 \\
\hline 64 & 55350776431903243 & 1197 & 1401 \\
\hline 65 & 80873624627234849 & 1219 & 1429 \\
\hline 66 & 203986478517455989 & 1223 & 1498 \\
\hline 67 & 218034721194214273 & 1247 & 1503 \\
\hline 68 & 305405826521087869 & 1271 & 1528 \\
\hline 69 & 352521223451364323 & 1327 & 1539 \\
\hline 70 & 401429925999153707 & 1355 & 1549 \\
\hline 71 & 418032645936712127 & 1369 & 1552 \\
\hline 72 & 804212830686677669 & 1441 & 1603 \\
\hline 73 & 1425172824437699411 & 1475 & 1647 \\
\hline 74 & 5733241593241196731 & 1487 & 1759 \\
\hline 75 & 6787988999657777797 & 1509 & 1773 \\
\hline 76 & 15570628755536096243 & 1525 & 1841 \\
\hline 77 & 17678654157568189057 & 1529 & 1852 \\
\hline 78 & 18361375334787046697 & 1549 & 1855 \\
\hline & The 1 & id & \\
\hline
\end{tabular}

